\title{
USO DE LAS CONDICIONES DE SALTO PARA EL MODELADO DE PROCESOS DE REACCIÓN Y SEPARACIÓN INTEGRADOS
}

Tesis que presenta

Ing. Ambiental Gustavo Aparicio Mauricio

Para la obtención del grado de

MAESTRO EN CIECIAS

(INGENIERÍA QUÍMICA) 


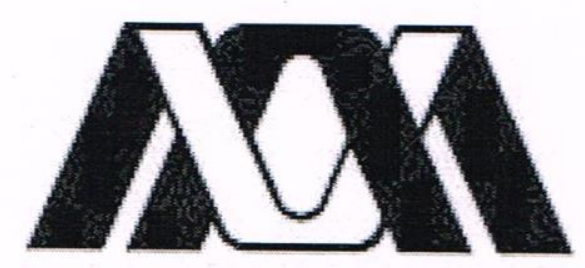

Casa abierta al tiempo

Universidad Autónoma Metropolitana - Iztapalapa

División de Ciencias Básicas e Ingeniería

Departamento de Ingeniería de Procesos e Hidráulica

Titulo:

USO DE LAS CONDICIONES DE SALTO PARA EL

MODELADO DE PROCESOS DE REACCIÓN Y SEPARACIÓN INTEGRADOS

Tesis que presenta

Ing. Ambiental Gustavo Aparicio Mauricio

Para la obtención del grado de

MAESTRO EN CIECIAS (INGENIERÍA QUÍMICA)

Asesor:

Dr. Jesús Alberto Ochoa Tapia

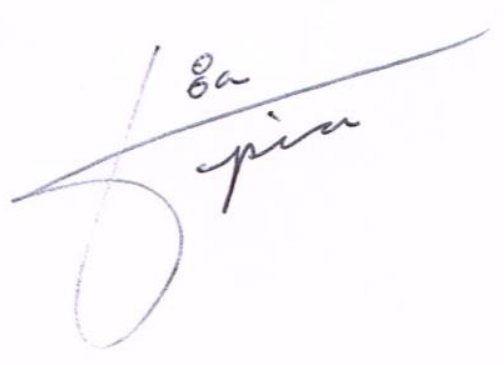




\section{AGRADECIMIENTOS}

Agradezco el apoyo económico otorgado por el Consejo Nacional de Ciencia y Tecnología (CONACYT) para el desarrollo de mis estudios de maestría.

Agradezco el apoyo económico otorgado por la Universidad Autónoma Metropolitana (UAM) y el Instituto de Ciencia y Tecnología del Distrito Federal (IC y TDF) para la conclusión de mis estudios de maestría. 


\section{Agradecimientos}

Agradezco a mis padres Julio y Julia por el apoyo incondicional y demostrarme que nada es inalcanzable

A mis hermanos Beatriz, Alejandro, Manuel y David

A mi asesor, el Dr. Ochoa por siempre compartir parte de su tiempo para discutir mis inquietudes

A mis sinodales, el Dr. Mauricio Sales, el Dr. Hugo Jiménez y el Dr. José Álvarez, quienes aportaron importantes comentarios y sugerencias al trabajo.

A mis amigos y al GTIIQ 


\section{RESUMEN}

Para el análisis preliminar del comportamiento de los procesos que integran reacción y separación, se utilizan datos experimentales en conjunto con una simulación numérica basada en modelos matemáticos, con la finalidad de determinar la operación adecuada y los parámetros de diseño del sistema que incluye, dos o más fases. En el modelo se considera el efecto de los fenómenos de transporte acoplados con reacciones químicas o biológicas de una fase, cuya longitud característica, es mucho menor que las asociadas con las otras fases. Esta situación complica la solución del modelo, ya que al resolver la ecuación diferencial parcial gobernante de esta fase y las condiciones de frontera asociadas, se requiere una malla computacional con un gran número de nodos. Por lo tanto una posible alternativa de modelado de este tipo de sistemas, es reemplazar las ecuaciones diferenciales correspondientes a tal zona, por condiciones de frontera o salto.

En este trabajo se analiza la validez del reemplazo de zonas con longitud pequeña por una superficie singular para un sistema en el que existe transporte de un soluto a través de una región formada por dos fases homogéneas, con el objetivo de encontrar la condición de salto, que reemplace a la fase donde ocurre difusión y reacción de manera simultánea. Utilizando conceptos matemáticos sencillos como las expansiones en series de Fourier y la identidad de Green, se desarrolla una solución analítica para el problema que considera una reacción lineal en una de las fases. Dicha solución es usada junto con dos funcionalidades (con respecto al tiempo) de la concentración en el seno del fluido situado a un lado de la región. Los resultados muestran que el tipo de funcionalidad en el tiempo no afecta la diferencia entre los fluxes de masa obtenidos con el modelo original y el que considera el salto. Adicionalmente se considera el análisis de dos casos no lineales donde también se explora el error que provoca el utilizar la condición de salto en el modelado. 


\section{ÍNDICE}

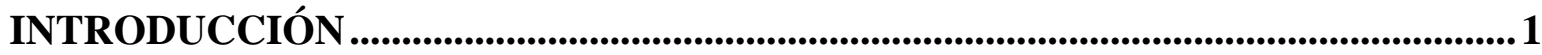

1.1 Intensificación de procesos...................................................................................... 1

1.2 Modelado de los procesos integrados de reacción y separación.....................................5

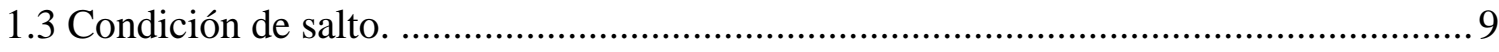

OBJETIVOS …..................................................................................................................11

1.4 Objetivo General............................................................................................... 11

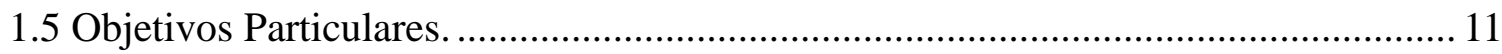

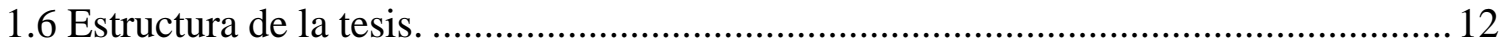

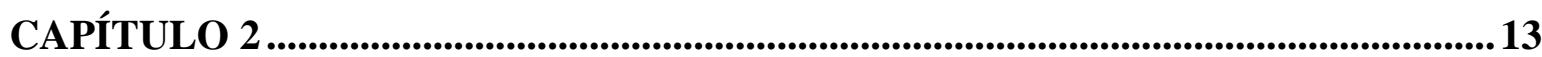

TRANSFERENCIA DE MASA EN UN SISTEMA DE DOS FASES ..............................13

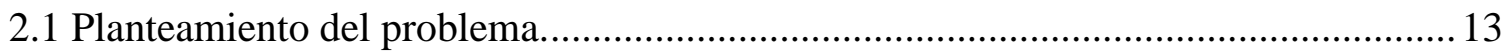

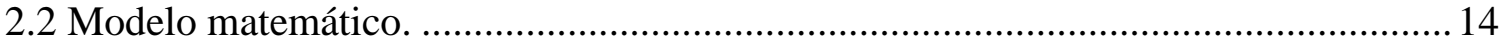

2.3 Metodología para derivar la condición de salto...................................................... 17

2.4 Metodología de solución........................................................................................... 20

2.4.1 Generación y solución del problema de Sturm Liouville.......................................21

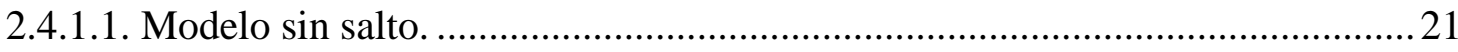

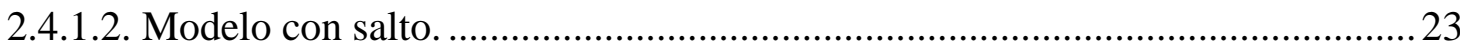

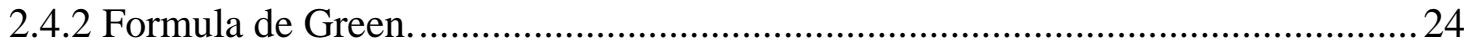

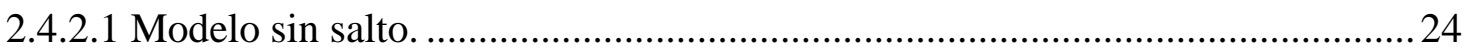

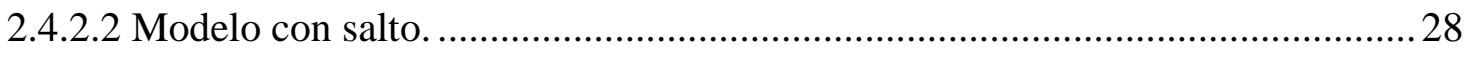

2.5 Definición del flux de masa. .................................................................................... 31

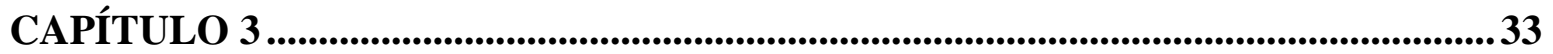

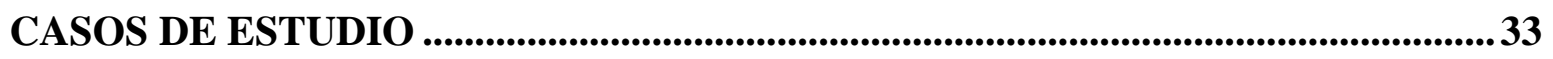

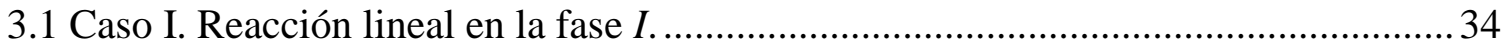

3.1.1 Caso I-a. La concentración en el seno del fluido externo a la fase $\eta$ es constante. 
3.1.2 Caso I-b. La concentración en el seno del fluido externo a la fase $\eta$ depende

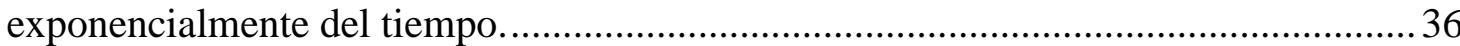

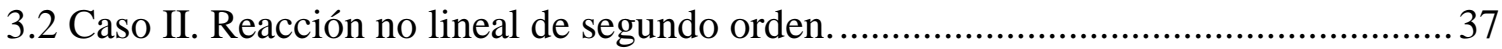

3.3 Caso III. Cinética de Michaelis Menten. .................................................................. 38

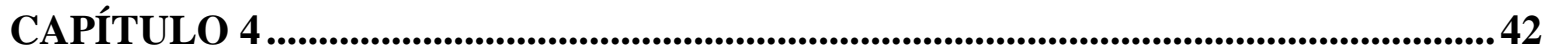

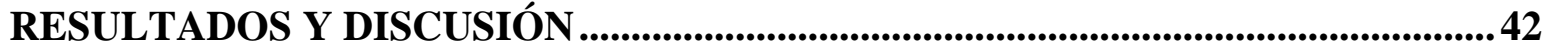

4.1 Caso I-a. La concentración en el seno del fluido externo a la fase $\eta$ es constante. ....43

4.2 Caso I-b. La concentración en el seno del fluido externo a la fase $\eta$ depende

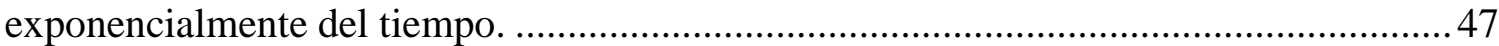

4.3 Caso II. Reacción no lineal de segundo orden......................................................49

4.4 Caso III. Cinética de Michaelis Menten. ................................................................. 52

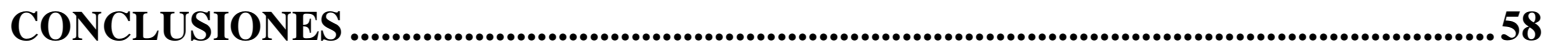

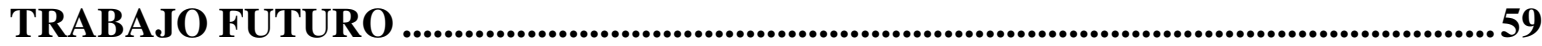

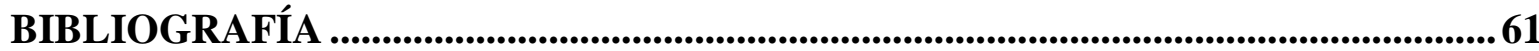

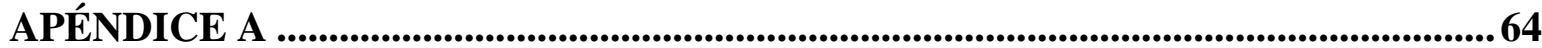

Detalles de la adimensionalizacion del sistema de dos fases .......................................... 64

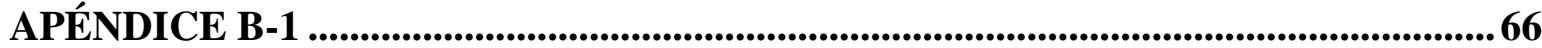

Detalles de la solución del problema de Sturm-Liouville para los dos modelos ............66

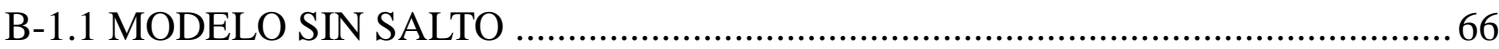

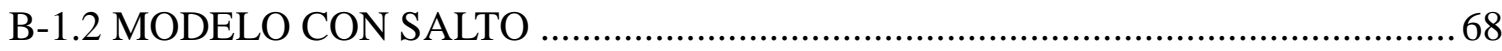

APÉNDICE B-2 ....................................................................................................................70

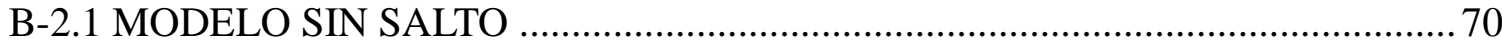

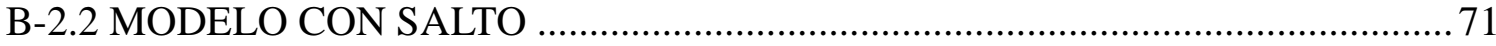

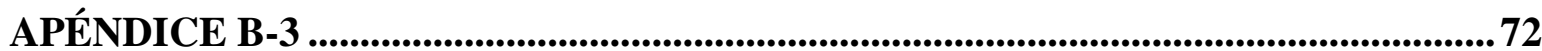

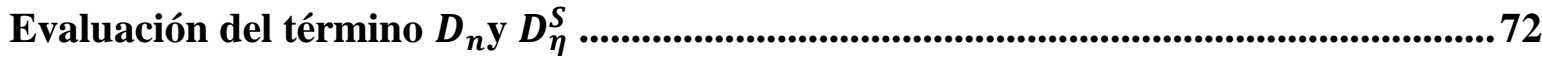


B-3.1 MODELO SIN SALTO ………………………………………………….....

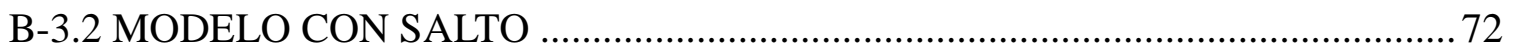

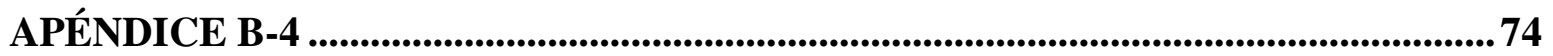

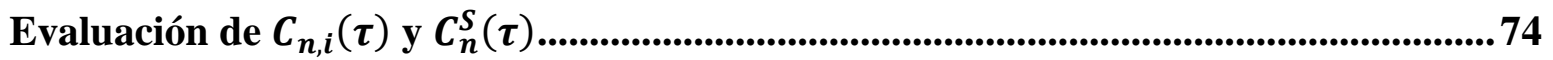

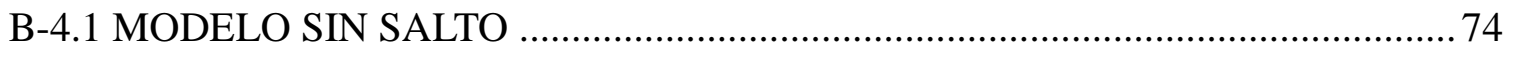

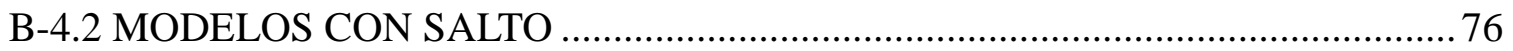

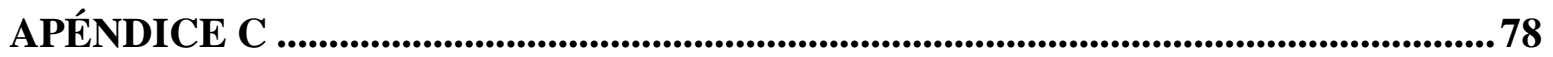

DIAGRAMAS DE FLUJO …......................................................................................................78

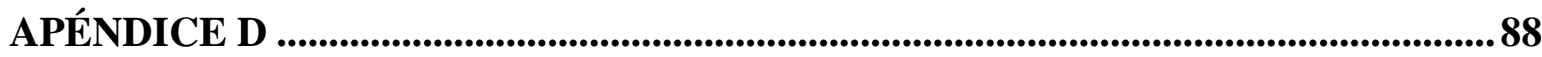

Búsqueda de los valores propios para el problema lineal.......................................................88

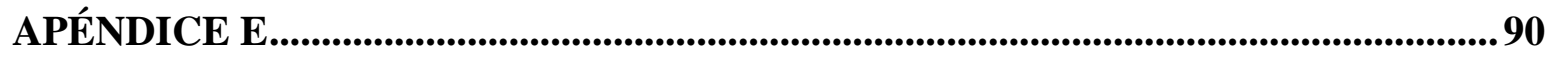

Deducción de la solución compuesta mediante el método de superposición ...................90 


\section{ÍNDICE DE FIGURAS}

Figura 1.1 Diagrama esquemático de una etapa en no equilibrio.................................... 7

Figura 2.1 Sistema formado por dos fases con coeficientes de difusión diferentes............ 13

Figura 3.1 Grafica de $V_{0}$ en función de $U_{I}$ para una reacción catalizada por una enzima....40

Figura $4.1 \%$ Error en función de $Y_{\eta}$ a diferentes valores de $\Phi_{\mathrm{I}}^{2}$. Caso I-a. ......................45

Figura $4.2 Y_{\eta}$ requerida para mantener un $\%$ Error de 1.0, en función de $\Phi_{\mathrm{I}}^{2}$ Caso I-a. .......45

Figura 4.3 Evolución del flux de masa evaluado en $Y=Y_{\eta}$ para el Caso I-a, manteniendo

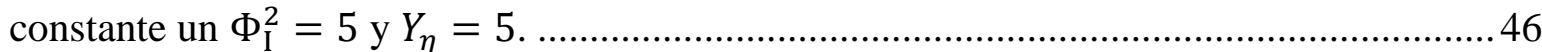

Figura 4.4 Evolución del flux de masa evaluado en $Y=Y_{\eta}$ para el Caso I-a, manteniendo

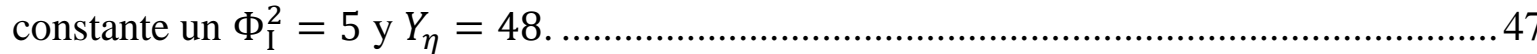

Figura 4.5 Evolución del flux de masa evaluado en $Y=Y_{\eta}$ para el Caso I-b, manteniendo constante un $\Phi_{\mathrm{I}}^{2}=5$ y $Y_{\eta}=5$.

Figura 4.6 Evolución del flux de masa evaluado en $Y=Y_{\eta}$ para el Caso I-b, manteniendo constante un $\Phi_{\mathrm{I}}^{2}=5$ y $Y_{\eta}=48$

Figura $4.7 \%$ Error en función de $Y_{\eta}$ a diferentes valores de $\Phi_{\mathrm{I}}^{2}$. Sistema no lineal de segundo orden

Figura $4.8 Y_{\eta}$ requerida para mantener un \%Error de 1.0, en función de $\Phi_{\mathrm{I}}^{2}$ Sistema no lineal de segundo orden.

Figura 4.9 Evolución del flux de masa evaluado en $Y=Y_{\eta}$ para el sistema no lineal de segundo orden, manteniendo constante un $\Phi_{\mathrm{I}}^{2}=5$ y $Y_{\eta}=5$.

Figura 4.10 Evolución del flux de masa evaluado en $Y=Y_{\eta}$ para el sistema no lineal de segundo orden, manteniendo constante un $\Phi_{\mathrm{I}}^{2}=5$ y $Y_{\eta}=34$.

Figura 4.11 Grafica de $V_{0}$ en función de $U_{I}$ para una reacción catalizada por una enzima diferentes valores de $\kappa_{M}$.

Figura $4.12 \%$ Error en función de $Y_{\eta}$ a diferentes valores de $\Phi_{\mathrm{I}}^{2}$. Sistema que considera una cinética de Michaelis Menten. 
Figura $4.13 Y_{\eta}$ requerida para mantener un \%Error de 1.0, en función de $\Phi_{\mathrm{M}}^{2}$ Sistema que considera una cinética de Michaelis Menten.

Figura 4.14 Evolución del flux de masa en $Y=Y_{\eta}$ para el sistema que considera una cinética de Michaelis Menten, manteniendo constante un $\Phi_{\mathrm{M}}^{2}=1$ y $Y_{\eta}=5$.

Figura 4.15 Evolución del flux de masa en $Y=Y_{\eta}$ para el sistema que considera una cinética de Michaelis Menten, manteniendo constante un $\Phi_{\mathrm{M}}^{2}=1$ y $Y_{\eta}=54 \ldots \ldots \ldots \ldots \ldots \ldots \ldots . . .56$

Figura C.1 Diagrama de Flujo para evaluar la solución analítica del MSS...........................78

Figura C.2 Diagrama de Flujo para evaluar la solución analítica del MCS..........................79

Figura C.3 Esquema de la celda computacional utilizada para la solución del sistema de dos fases sin salto.

Figura C.4 Diagrama de Flujo para evaluar la solución numérica en estado estacionario del MSS. 83

Figura C.5 Diagrama de Flujo para evaluar la solución numérica en estado transitorio del MSS

Figura C.6 Diagrama de Flujo para evaluar la solución numérica en estado estacionario del MCS

Figura C.7 Diagrama de Flujo para evaluar la solución numérica en estado transitorio del MCS

Figura D.1 Evolución de la función que determina los valores propios para el MSS, para tres valores del $\Phi_{I}^{2}$, manteniendo $Y_{\eta}=5$.

Figura D.2 Evolución de la función que determina los valores propios para el MCS, para tres valores del $\Phi_{\mathrm{I}}^{2}$, manteniendo $Y_{\eta}=5$. 


\section{ÍNDICE DE TABLAS}

Tabla 2.1 Definición de variables adimensionales.

Tabla 2. 2 Definición de parámetros adimensionales................................................. 17

Tabla 3.1 Coeficientes $C_{n, i}(\tau)$ y $D_{n}$ para el MSS y $C_{\eta}^{S}(\tau)$ y $D_{n}^{S}$ para el MCS.................34

Tabla 4.1 Valores de los parámetros adimensionales utilizados en las soluciones de los

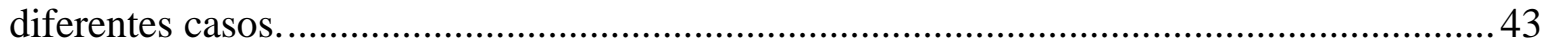

Tabla 4.2 Tiempos de cómputo utilizados para la evaluación en estado transitorio de los dos modelos. Caso II. .................................................................................................. 52

Tabla 4.3 Tiempos de cómputo utilizados para la evaluación en estado transitorio de los dos

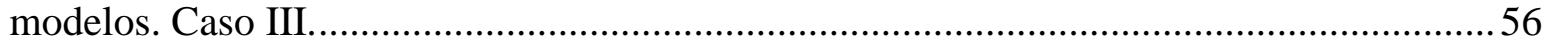

Tabla 4.4 Tiempos de cómputo utilizados para la evaluación en estado estacionario de los dos modelos. 


\section{NOMENCLATURA}

$C_{i} \quad$ Concentración del soluto en la $i$-ésima fase, $\mathrm{mol} / \mathrm{m}^{3}$.

$C_{\eta}^{F} \quad$ Concentración del soluto alrededor de la fase $\eta, \mathrm{mol} / \mathrm{m}^{3}$.

$C_{i}^{e} \quad$ Concentración del soluto en equilibrio de la $i$-ésima fase, $\mathrm{mol} / \mathrm{m}^{3}$.

$C_{i 0}(y) \quad$ Condición de distribución inicial en la $i$-ésima fase, $\mathrm{mol} / \mathrm{m}^{3}$.

$C_{n, i}(\tau) \quad$ Función definida en la ecuación (2.57), para la $i$-ésima fase, adimensional.

$C_{n}^{S}(\tau) \quad$ Función definida en la ecuación (2.73), para la fase $\eta$ del modelo con salto, adimensional.

$D_{i} \quad$ Difusividad molecular de la $i$-ésima fase, $\mathrm{m}^{2} / \mathrm{s}$.

$D^{*} \quad$ Difusividad molecular de referencia, $\mathrm{m}^{2} / \mathrm{s}$.

$D_{n} \quad$ Función definida en la ecuación (2.52), adimensional.

$D_{n}^{s} \quad$ Función definida en la ecuación (2.68), para el modelo con salto, adimensional.

$f_{\eta}(t) \quad$ Funcionalidad en el tiempo de la concentración del soluto alrededor de la fase $\eta, \mathrm{mol} / \mathrm{m}^{3}$.

$F_{\eta}(\tau) \quad$ Funcionalidad en el tiempo de la concentración del soluto alrededor de la fase $\eta$, adimensional.

$I_{n}(\tau) \quad$ Función definida en la ecuación (2.51), adimensional

$I_{n}^{s}(\tau) \quad$ Función definida en la ecuación (2.67), para el modelo con salto, adimensional.

$K_{e q}^{\eta I} \quad$ Constante de equilibrio entre las dos fases.

$k_{I} \quad$ Constante de velocidad para una reacción lineal, $\mathrm{s}^{-1}$.

$K_{I} \quad$ Constante de velocidad para una reacción de segundo orden, $\mathrm{m}^{3} / \mathrm{s}$ mol.

$K_{M} \quad$ Constante de Michaelis Menten, $\mathrm{mol} / \mathrm{m}^{3}$. 
$L_{I} \quad$ Longitud característica de la fase $I, \mathrm{~m}$.

$L_{\eta} \quad$ Longitud característica de la fase $\eta, \mathrm{m}$.

$N_{\eta} \quad$ Flux de masa en $Y=Y_{\eta}, \mathrm{mol} / \mathrm{s} \mathrm{m}^{2}$.

$\mathrm{N}_{\eta} \quad$ Flux de masa en $Y=Y_{\eta}$, adimensional.

$N \quad$ Numero de incrementos totales.

$N_{I} \quad$ Numero de incrementos para la fase $I$.

$r \quad$ Parámetro definido por la ecuación (C.10), adimensional.

$R^{\eta} \quad$ Parámetro definido por la ecuación (C.4), adimensional.

$R^{I} \quad$ Parámetro definido por la ecuación (C.13), adimensional.

$t \quad$ Tiempo, s.

$U_{i} \quad$ Concentración del soluto en la $i$-ésima fase, adimensional.

$U_{\eta}^{1} \quad$ Concentración constante del soluto, $1 \mathrm{~mol} / \mathrm{m}^{3}$.

$U_{i 0}(Y) \quad$ Condición de distribución inicial en la $i$-ésima fase, adimensional.

$\left\{U_{I}\right\} \quad$ Concentración promedio del soluto en la fase $I$, adimensional.

$V_{\max } \quad$ Velocidad máxima de una reacción enzimática, $\mathrm{mol} / \mathrm{m}^{3} \mathrm{~s}$.

$v_{0} \quad$ Velocidad de reacción enzimática: Michaelis Menten, $\mathrm{mol} / \mathrm{m}^{3} \mathrm{~s}$.

$V_{0} \quad$ Velocidad de reacción enzimática: Michaelis Menten, adimensional.

y Posición, m.

Y Posición, adimensional.

$Y_{\eta} \quad$ Relación entre las longitudes características de cada fase.

\section{Letras griegas}

$\alpha_{i} \quad$ Relación del coeficiente de difusión de la $i$-ésima fase con la de referencia.

$\beta_{\eta} \quad$ Factor de decaimiento, $\mathrm{s}^{-1}$.

$\delta_{i} \quad$ Relación entre los coeficientes de difusión de la i-ésima fase y la longitud 
característica de cada fase.

$\kappa_{\eta} \quad$ Relación entre los coeficientes de difusión de cada fase y la constante de equilibrio.

$\kappa_{M} \quad$ Constante de Michaelis Menten, adimensional.

$\lambda_{\mathrm{j}} \quad$ Vector de los valores propios.

$\lambda_{\mathrm{j}}^{\mathrm{s}} \quad$ Vector de los valores propios para el modelo con salto.

$\mu_{i}^{2} \quad$ Relación entre los valores propios y los coeficientes de difusión de la $i$-ésima fase.

$\left(\mu_{i}^{s}\right)^{2} \quad$ Relación entre los valores propios y los coeficientes de difusión de la $i$-ésima fase para el modelo con salto.

$\xi \quad$ Posición con respecto a la longitud característica de la fase $\eta$, adimensional.

$\sigma_{\eta} \quad$ Parámetro pre-exponencial, $\mathrm{mol} / \mathrm{m}^{3}$.

$\Phi_{I}^{2} \quad$ Modulo de Thiele,

$\Phi_{M}^{2} \quad$ Modulo de Thiele para una cinética de Michaelis Menten

$\varphi_{i} \quad$ Funciones propias de la $i$-ésima fase.

$\varphi_{\eta}^{s} \quad$ Función propia de la fase $\eta$ para el modelo con salto.

$\tau \quad$ Tiempo, adimensional.

\section{Subíndices}

$i \quad$ Indica la i-ésima fase $(i=\eta, I)$.

$0 \quad$ Condición inicial.

j Indica el j-ésimo valor propio

\section{Superíndices}

e Equilibrio.

$s \quad$ Perteneciente al Modelo con Salto. 


\section{CAPÍTULO 1}

\section{INTRODUCCIÓN}

\subsection{Intensificación de procesos.}

Tanto las necesidades económicas, como las demandas ecológicas son una fuerza importante que impulsa la mejora de los procesos y plantas químicas. Para cumplir estos objetivos, los procesos deben ser intensificados, con la finalidad de obtener productos de alta calidad, aumentar el rendimiento mediante la reducción o incluso la supresión de subproductos y disminuir el consumo de energía. La intensificación de procesos (IP) es un término usado para describir la estrategia de hacer reducciones sustanciales en el tamaño físico de una planta química, donde se busca lograr un objetivo de producción (Dautzenberg y Mukherjee, 2001). La IP ha sido aplicada por la industria de procesos químicos para reducir las inversiones y los costos de operación de las plantas químicas, con la finalidad de incrementar la rentabilidad del proceso y mitigar las emisiones de gases invernadero.

La intensificación de procesos comprende equipo novedoso, tecnologías de procesamiento y métodos de desarrollo de procesos que, comparados con los métodos convencionales, ofrecen mejoras sustanciales en manufactura y procesamiento de compuestos químicos o biológicos. La integración de reacción y separación en una sola unidad es uno de los métodos más importantes de intensificación de procesos. Existen procesos de integración para sistemas de gas-líquido, gas-sólido, líquido-sólido, así como líquido-líquido que cubren un amplio rango de aplicaciones en ingeniería química. 
Los procesos integrados de reacción y separación en un solo equipo (PIRS) ofrecen la ventaja de reducir los costos en equipo y energía; además, esto quizá lleve al incremento de la eficiencia del proceso global con respecto al consumo de reactantes desde la conversión y selectividad del sistema de reacción (Schmidt-Traub y Górak, 2006). Debido a la demanda de procesos de producción más eficientes, el uso de PIRS ha sido extendido a otros sistemas de reacción. Los PIRS son frecuentemente llamados reactores multifuncionales.

Los reactores multifuncionales son aquellos que en un solo equipo, además de la reacción, ocurre otras operaciones de manera simultáneamente (Westerterp, 1992 ; Agar, 1999). Estas operaciones pueden ser, procesos de transferencia de calor, masa y momento o incluso otra reacción independiente. Dautzenberg (2001) propuso la siguiente clasificación de reactores multifuncionales en base al nivel donde ocurre la integración de las funcionalidades;

- Tipo A: la multifuncionalidad ocurre al nivel del catalizador.

- Tipo B: la multifuncionalidad ocurre en la reacción interfacial.

- Tipo C: la multifuncionalidad ocurre al nivel dentro del reactor.

- Tipo D: la multifuncionalidad ocurre al nivel fuera del reactor.

En el tipo C, la reacción ocurre simultáneamente con una operación unitaria dentro del reactor, las principales ventajas de considerar la separación in situ de productos en la zona de reacción son: mejorar la conversión en reacciones que están limitadas por el equilibrio, mediante el desplazamiento del equilibrio hacia los productos, evitar en mayor medida las reacciones de productos indeseables mejorando la selectividad e incrementar la velocidad de reacción de productos inhibidos. La destilación catalítica (DC), la absorción reactiva (AR), la extracción reactiva (ER), la cromatografía liquida reactiva (CLR) y los reactores con membrana (RM) se ubican dentro de este nivel (Dautzenberg y Mukherjee, 2001).

La DC es una combinación de reacciones químicas con catalizadores heterogéneos y la separación vía destilación. Esto está en contraste con los procesos de destilación reactiva en 
que usualmente la catálisis homogénea es aplicada. Tanto la inversión como los costos de operación de la DC son mucho menores a los utilizados en procesos convencionales de reacción seguidos por destilación.

Las tecnologías CDMtbe, CDTame and CDEtbe, utilizan la esterificación catalítica de alcanos con un alcohol para producir éteres de alta calidad. Actualmente, existen más de 50 unidades de CDTECH operando alrededor del mundo. La tecnología de la DC es ampliamente usada en la alquilación de aromáticos, remoción de benceno de las fracciones reformadas, desulfuración selectiva de fracciones de gasolina, así como de varias hidrogenaciones selectivas (Dautzenberg y Mukherjee, 2001).

La AR representa una caso especial de reactores multifuncionales, donde los procesos que son afectados por un sistema de reacciones químicas son acoplados con una unidad de separación vía adsorción. Esta propuesta puede ser adicionalmente extendida para reacciones gas-sólido, como un método selectivo para la manipulación del perfil de concentración de una especie en particular. En este sentido, uno o más componentes pueden ser removidos por adsorción y suministrados por desorción debido a la mezcla de reacción, esto afectara la cinética y termodinámica del sistema reaccionante. Una aplicación común de la $\mathrm{AR}$ es el aumento de la conversión en una reacción reversible simple, superando las limitaciones termodinámicas por la selectividad de remoción de un subproducto. Elsner y col. (2002) evaluaron la capacidad de aumentar la conversión en dos sistemas de reacción en fase gas catalizadas heterogéneamente, la reacción Claus usada en la recuperación de azufre y la recuperación directa de cianuro de hidrogeno a partir de monóxido de carbono y amoniaco, mediante AR.

La ER es aplicada en el reciclado de catalizadores y además tiene cierta influencia en la selectividad de la reacción, su principio es desarrollar la reacción en una fase, mientras que en la segunda fase, que es catalíticamente inactiva solo contener los reactantes y productos. La ER es ampliamente utilizada para la purificación de solventes, la extracción de productos y la separación selectiva de componentes físicamente similares. Además es 
aplicado en la separación de metales o farmacéuticos, tratamiento de agua residual o la purificación de mezclas orgánicas.

Un interesante ejemplo de ER es la separación de plutonio y uranio de los residuos nucleares mediante el proceso PUREX. Otro ejemplo industrial es la desulfuración del combustible utilizando el proceso de UPON-Merox y la extracción de Penicilina G Gaidhani y col., 2002).

La cromatografía es una técnica de separación altamente selectiva que principalmente es usada para la separación de componentes empleados como químicos finos, farmacéuticos, aditivos de alimentos y productos biológicos. El acoplamiento de reacciones químicas o bioquímicas y la separación por cromatografía conlleva a un sistema integrado para la producción de compuestos con un nivel de pureza alto. Dentro del reactor cromatografico, la conversión de los reactantes así como la separación de los productos ocurre de manera simultánea. Por lo tanto, las reacciones reversibles pueden superar la limitante por conversión establecida por el equilibrio químico. Las reacciones dentro de la CLR pueden ser catalizadas de manera homogénea o heterogénea. Estas últimas ocurren con mayor frecuencia, existen casos especiales, tal como la esterificación, donde la resina de intercambio iónico tiene doble función, como catalizador de la reacción y adsorbente para la separación (Mazzotti y col., 1996). Otras aplicaciones a escala laboratorio son, reacciones de hidrólisis, sacarificación, así como la preparación de reactivos inestables.

Un RM es un sistema que acopla reacción y separación de uno o más productos, donde el proceso de separación utiliza una membrana selectiva. Entre las ventajas que ofrecen los RM se encuentran; la habilidad de estos reactores de evitar las limitaciones termodinámicas de un proceso controlado por el equilibrio, permitiendo que a baja temperatura se obtenga la misma conversión de los reactantes o buscar una mayor conversión a la misma temperatura, así mismo al operar los reactores a baja temperatura se pueden incorporar nuevas estrategias de integración de calor, que van desde líneas de escape de turbina de gas hasta calentadores solares que utilizan sales fundidas como fluido de transporte de calor, o incluso helio de una planta nuclear (De Falco y col., 2011). 
Los RM son aplicados en algunos procesos de la industria petroquímica, entre los cuales se encuentran el reformado de vapor, oxidación parcial catalítica, reformado autotérmico, craqueo de $\mathrm{H}_{2} \mathrm{~S}$ y la deshidrogenación de hidrocarburos. Un interesante ejemplo de RM es la producción de hidrogeno mediante el metano reformado con vapor, utilizando una membrana de paladio con el objetivo de reducir la temperatura de operación de los reactores convencionales (Lin y col., 2003). La biotecnología es otra área donde los RM son de gran interés, aquí el sistema de separación por membrana es acoplada con reacciones biológicas, por ejemplo en una amplia variedad de fermentaciones para la producción de aminoácidos y antibióticos. También los RM se utilizan en el tratamiento biológico de aguas residuales (Sanchez Marcano y Tsotsis, 2002).

\subsection{Modelado de los procesos integrados de reacción y separación.}

En general, los PIRS son naturalmente multicomponente, esto significa que estos procesos son cualitativamente más complejos que los procesos binarios. El acoplamiento de la termodinámica y los procesos de difusión en las diferentes fases y en la interfase, que son de carácter multicomponente son acompañadas por reacciones químicas complejas (Kening y Górak, 1995). Además de los experimentos, la simulación numérica de los PIRS es de gran utilidad para un análisis preliminar de su comportamiento y la determinación de los parámetros de diseño. Por lo tanto para la simulación se requieren modelos matemáticos que sean capaces de tomar en consideración, la hidrodinámica de los equipos, las resistencias a la transferencia de masa y las cinéticas de las reacciones. En el modelado de estos procesos, resulta un sistema acoplado de ecuaciones diferenciales parciales no lineales. Debido a su complejidad, el sistema de ecuaciones diferenciales parciales que resultan del modelo matemático para los PIRS, generalmente son resueltos numéricamente. Las técnicas numéricas mayormente empleadas son:

- Métodos de discretización espacial, se utilizan para convertir las ecuaciones diferenciales parciales a ecuaciones diferenciales ordinarias, una vez obtenidas se procede a resolver las ecuaciones que describen el sistema dinámico, mediante un algoritmo estándar de solución de sistemas de ecuaciones diferenciales ordinarias. 
- Métodos de discretización en espacio y tiempo, convirtiendo las ecuaciones diferenciales parciales en un sistema de ecuaciones algebraicas no lineales, que puede resolverse por métodos comunes tal como el algoritmo de Newton.

Con la discretización en espacio y tiempo se obtienen un número elevado de sistemas de ecuaciones, donde el tiempo de cálculo y los requerimientos de memoria son fuertemente influenciados por el número de intervalos de discretización. Debido a ello, métodos de discretización efectivos son necesarios, entre los cuales se encuentran, el método de refinamiento de la malla (Nowak y col., 1996) en regiones donde los gradientes son bruscos o también se puede utilizar métodos de discretizacion no lineales.

La factibilidad de un modelo sofisticado de PIRS es cuestionable si los problemas de diseño de la planta, tales como los modelos basados en control y la optimización de los procesos en línea, no han sido resueltos. En este caso un modelo reducido de manera razonable debe ser aplicado (Kreul y col., 1999). Por ejemplo al modelar los procesos de DC y AR a escala industrial, una apropiada subdivisión de las columnas en elementos más pequeños es usualmente necesaria. Estos elementos, llamados etapas, son identificados como platos reales o segmentos de una columna empacada. Estos pueden ser descritos utilizando diferentes modelos teóricos, con un amplio rango de restricciones fisicoquímicas y de precisión, entre los cuales se encuentra el modelo de la etapa en equilibrio y el método de aproximación basado en velocidades.

La aproximación basada en velocidades es un modelo físicamente más consistente que el modelo de etapa en equilibrio, para describir una etapa de una columna (Seader, 1989). Esta aproximación implica que las velocidades actuales de transferencia de masa multicomponente y de calor, así como las velocidades de las reacciones químicas, se toman en cuenta directamente. La transferencia de masa en la interfase gas-líquido puede ser descrita utilizando diferentes conceptos teóricos, los métodos mayormente utilizados son el modelo de las dos películas Lewis y Whitman, 1924) y el modelo de renovación de superficie, debido en que ambos modelos los parámetros utilizados son estimados vía correlaciones experimentales. 
El modelado de dos películas considera que todas las resistencias a la transferencia de masa están concentradas en las películas adyacentes a la intefase y que la transferencia por difusión molecular ocurre solamente dentro de las películas, como se muestra en la Figura 1.1. Fuera de las películas, en los fluidos, el nivel de mezclado es tan alto que no existe un gradiente de concentración, por lo tanto el transporte por difusión es en una solo dimensión y normal a la interfase. La difusión multicomponente en las películas puede ser descrita por las ecuaciones de Maxwell-Stefan, que pueden ser derivados por la teoría cinética de los gases (Noeres y col., 2003). Para algunos procesos de DR, la reacción química ocurre solamente en la fase líquida, por lo tanto el balance de masa en la película líquida considera un termino de reacción, mientras que en la película de gas solo el de difusión.

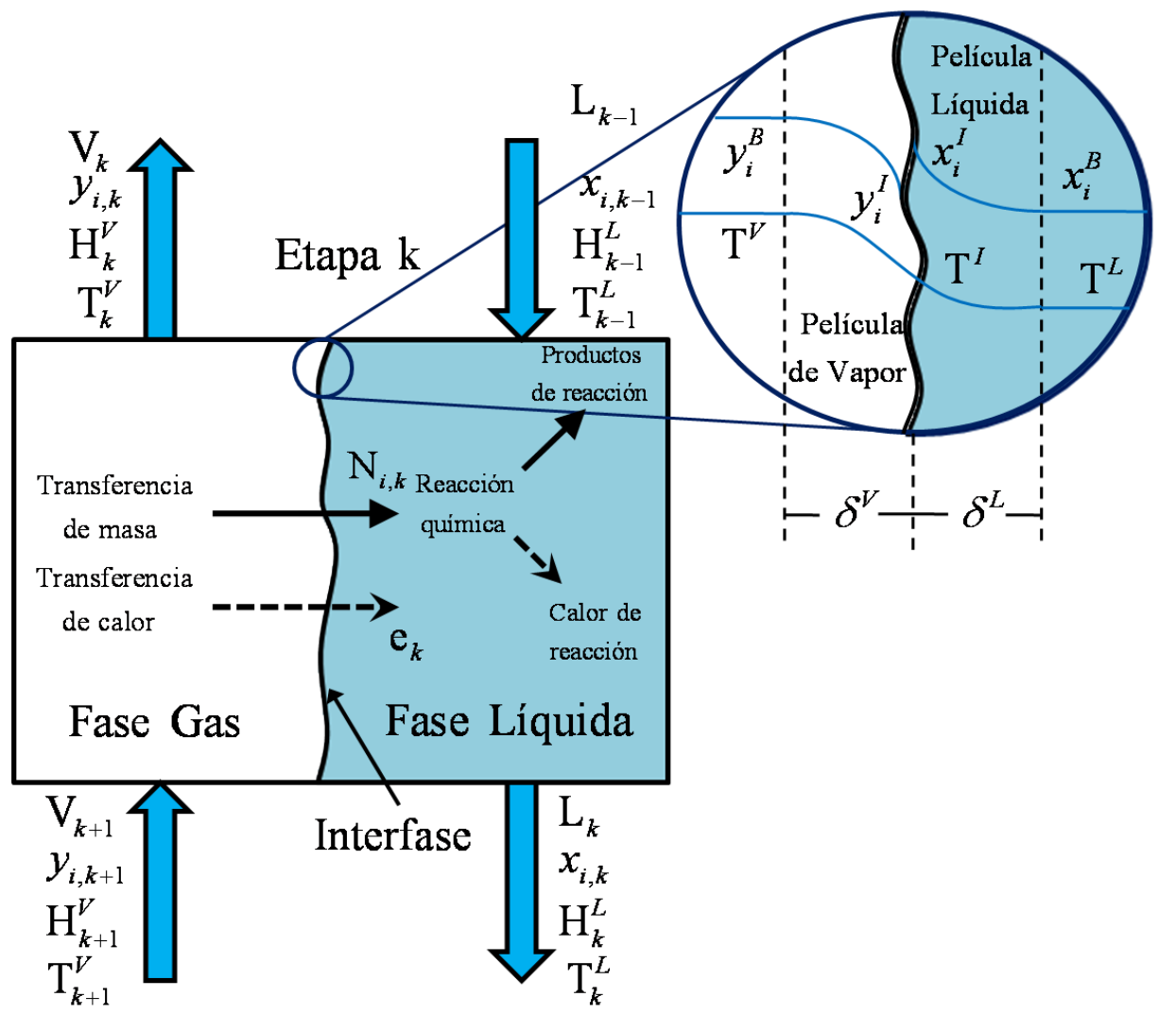

Figura 1.1 Diagrama esquemático de una etapa en no equilibrio. 
Por otro lado, en algunos casos la longitud característica de una fase en donde suceden los fenómenos importantes dentro del equipo del PIRS es mucho menor que las asociadas con las de otros elementos del sistema. Como ejemplos de longitudes pequeñas, se encuentran los trabajos de Asprion (2006) quien estudió la transferencia de calor en una columna seccionada donde fluye nitrógeno y agua a contracorriente, el grosor de la película líquida de conducción fue de $6 \times 10^{-6} \mathrm{~m}$, que es 33 veces menor que el grosor de la película gaseosa de conducción. Además, el modelo de dos películas estableció que la película líquida de conducción es de 2 a 5 veces mayor que el grosor de la película líquida de difusión $\left(\delta^{L}\right)$ y para los gases, ambas películas tienen el mismo grosor. Por lo tanto el grosor de la película gaseosa de difusión $\left(\delta^{V}\right)$ fue de 166-66 veces mayor que la película líquida de difusión $\left(\delta^{L}\right)$, pero al incluir una reacción rápida en la fase líquida, la transferencia de materia ocurre solamente en la película líquida de difusión y su espesor disminuye.

Otro ejemplo es de Torres M. y col.(1994), quienes desarrollaron un modelo para simular un reactor con membrana catalítica de tres fases utilizado para la hidrogenación de nitrobenceno, el sistema está compuesto por un reactor tubular que contiene a la membrana de alúmina con un grosor de $2.5 \times 10^{-6} \mathrm{~m}$ y el soporte adyacente a la membrana que tiene un grosor de $1.6 \times 10^{-3} \mathrm{~m}$, que es 640 veces mayor al grosor de la membrana. El modelo incluye la transferencia de masa por difusión en el soporte y adicionalmente en la membrana se incorpora el efecto de una reacción química.

Longitudes pequeñas de las regiones donde suceden los fenómenos importantes dentro del equipo del PIRS, complican la solución del modelo, que llevará eventualmente al dimensionamiento de las diferentes unidades necesarias en un proceso. Esto porque la solución numérica de una ecuación diferencial en una región de cambios bruscos de las variables requiere una malla computacional con un número elevado de nodos. 
Lo anterior motiva a estudiar otras alternativas de modelado, una posible es el reemplazo de zonas con longitud característica pequeña por una superficie singular; esto es, las ecuaciones diferenciales correspondientes a tal zona se reemplazan por condiciones de frontera apropiadas. Procedimientos similares se han aplicado en la deducción de modelos para sistemas de varias fases, para el desarrollo de condiciones de salto de flux o concentración para el acoplamiento de ecuaciones de medio efectivo. Para este tipo de casos la metodología ya está claramente establecida y ha permitido el reemplazo de zonas de cambio abrupto de propiedades efectivas de medios porosos por condiciones de frontera para transporte de cantidad de movimiento, energía y masa (Valdés Parada y col., 2006, 2007, Aguilar Madera y col., 2011). El resultado son las llamadas condiciones de salto.

Hasta este punto se ha considerado una breve revisión de la literatura relacionada con los PIRS y su modelado, a continuación se expondrá brevemente algunos trabajos dedicados a obtener condiciones de frontera en la interfase, ya sean empíricas o metodológicas, donde se incluye la metodología para obtener la condición de salto.

\subsection{Condición de salto.}

El estudio de los fenómenos de transporte en sistemas multifásicos, ha sido un tema de interés en la comunidad científica desde el siglo XIX. Sin embrago, la mayor parte de los estudios, teóricos y experimentales, se han dedicado al estudio en las regiones homogéneas de dichos sistemas, dejando en segundo plano el análisis en la inter-región. Fue a partir del trabajo teórico-experimental de Beavers y Joseph (1967), que se despertó el interés en el estudio de los fenómenos de transporte en esta porción del sistema.

El desarrollo y análisis de condiciones de salto en sistemas multifásicos, en general se puede clasificar en tres categorías: la primera concierne a la condición de salto empírica de Beavers y Joseph (1967) y los trabajos que de ella se derivaron, entre los cuales resalta el análisis de Haber y Mauri (1983) que recalcan entre sus conclusiones la necesidad de condiciones de frontera particulares, para cuya deducción se necesita "algo más que la intuición física", que permitan que sus resultados sean factibles; la segunda se refiere a la 
obtención de condiciones de salto mediante la aplicación de la metodología propuesta por (Ochoa Tapia y Whitaker, 1995) .Estos dos tipos de condiciones de frontera se clasificaran como no cerradas, dado que están en función de parámetros ajustables; la tercera categoría en que se dividen las contribuciones se refiere a aquellas dedicadas a predecir el coeficiente de salto obtenido mediante la metodología de Ochoa-Tapia y Whitaker (1995), donde el trabajo de Wood y col. (2000), es crucial ya que desarrolla condiciones de salto cerradas, es decir, que no dependan de parámetros ajustables, las cuales involucran la solución de un problema de cerradura.

Los pasos claves de la metodología para obtener la condición de salto cerrada, con base a los trabajos de Ochoa-Tapia y Whitaker (1995) y Wood y col. (2000), se describen brevemente a continuación:

- Obtener, usando el método de promedio volumétrico, ecuaciones de medio efectivo, considerando; restricciones, que permitan que dichas ecuaciones sean validas en todo el sistema y que al imponer restricciones de escala se recuperen las ecuaciones válidas en las regiones homogéneas.

- Integrar las ecuaciones obtenidas en una región de promediado de volumen que incluya porciones de ambas regiones y su frontera. Posteriormente, se restan las ecuaciones válidas en las partes homogéneas de la que es válida en todo el dominio.

- Plantear el problema de cerradura restando las ecuaciones de transporte puntuales con las promedio y las condiciones de salto. Aquí se usa la descomposición espacial de Gray (1975) para la propiedad a estudiar y para el coeficiente que presente cambios espaciales.

- Resolver el problema de cerradura de manera análoga a la usada en la literatura relacionada con el promedio volumétrico (por ejemplo, Whitaker, 1999) y como consecuencia, definir el coeficiente efectivo para la inter-región que incluya la solución de dicho problema y por ultimo para obtener la forma cerrada de la condición de salto, sustituir la definición del coeficiente en la misma. 


\section{OBJETIVOS}

\subsection{Objetivo General.}

Estudiar un sistema unidimensional de separación entre una fase fluida y una fase líquida de espesor "pequeño", donde ocurre una reacción química, para determinar bajo qué condiciones es adecuado reemplazar las ecuaciones que representan el transporte en la fase líquida por una condición de frontera apropiada.

\subsection{Objetivos Particulares.}

- Contar con una solución analítica para evaluar la condición de salto que reemplace la ecuación de transporte de masa de la fase líquida, considerando una reacción lineal de primer orden y determinar bajo qué condiciones es válida esta condición de frontera.

- Evaluar la condición de salto que reemplace la ecuación de transporte de masa de la fase líquida, en dos problemas no lineales, mediante una solución numérica y determinar bajo qué condiciones es válida esta condición de frontera.

- Concluir sobre la validez del reemplazo de la ecuación gobernante de una fase por una condición de frontera. 


\subsection{Estructura de la tesis.}

Para lograr los objetivos planteados anteriormente, el presente trabajo se divide de la siguiente forma: en el capítulo dos se hace una descripción del sistema de estudio (sección 2.1), en la sección 2.2 se plantean las ecuaciones que gobiernan la transferencia de masa en un sistema formado por dos fases considerando que la concentración depende de una dimensión y del tiempo. En la sección 2.3 se describe los pasos a seguir para derivar la condición de salto para este tipo de modelos y en la sección 2.4 se desarrolla la metodología de solución y por ultimo en este capítulo se define el cálculo del flux de masa para concentraciones diluidas.

En el capítulo tres se plantean cuatro diferentes casos de estudio, en dos de ellos se considera diferentes funcionalidades de la concentración con respecto al tiempo en una de las fronteras de la fase que no considera la reacción. Además se consideran en los otros casos, dos problemas adicionales al sistema lineal, que incluyen una reacción no lineal en una de las fases.

En el capitulo cuatro se exponen los resultados para los casos lineales (sección 4.1 y 4.2), posteriormente en las secciones 4.3 y 4.4 , se discuten los resultados obtenidos a partir de una solución numérica para los problemas no lineales. Por último se presentan las conclusiones de este trabajo.

Finalmente después de presentar las referencias y la bibliografía, se concluye con una sección de apéndices en donde se presentan los detalles de la solución analítica, la obtención de los valores propios utilizados en la solución analítica y los diagramas de flujo de los métodos de solución; analítico y numéricos. 


\section{CAPÍTULO 2}

\section{TRANSFERENCIA DE MASA EN UN SISTEMA DE DOS FASES}

\subsection{Planteamiento del problema.}

Se tiene interés en resolver en forma analítica el problema transitorio de difusión del soluto $A$ a través de un sistema formado por dos fases homogéneas e isotrópicas con propiedades de transporte constantes en el espesor de cada fase. En la fase $\eta$ se considera solo la difusión y en la fase $I$ ocurre de manera simultánea el transporte por difusión y el aumentado por una reacción lineal de primer orden. Tal como se muestra en la Figura 2.1.

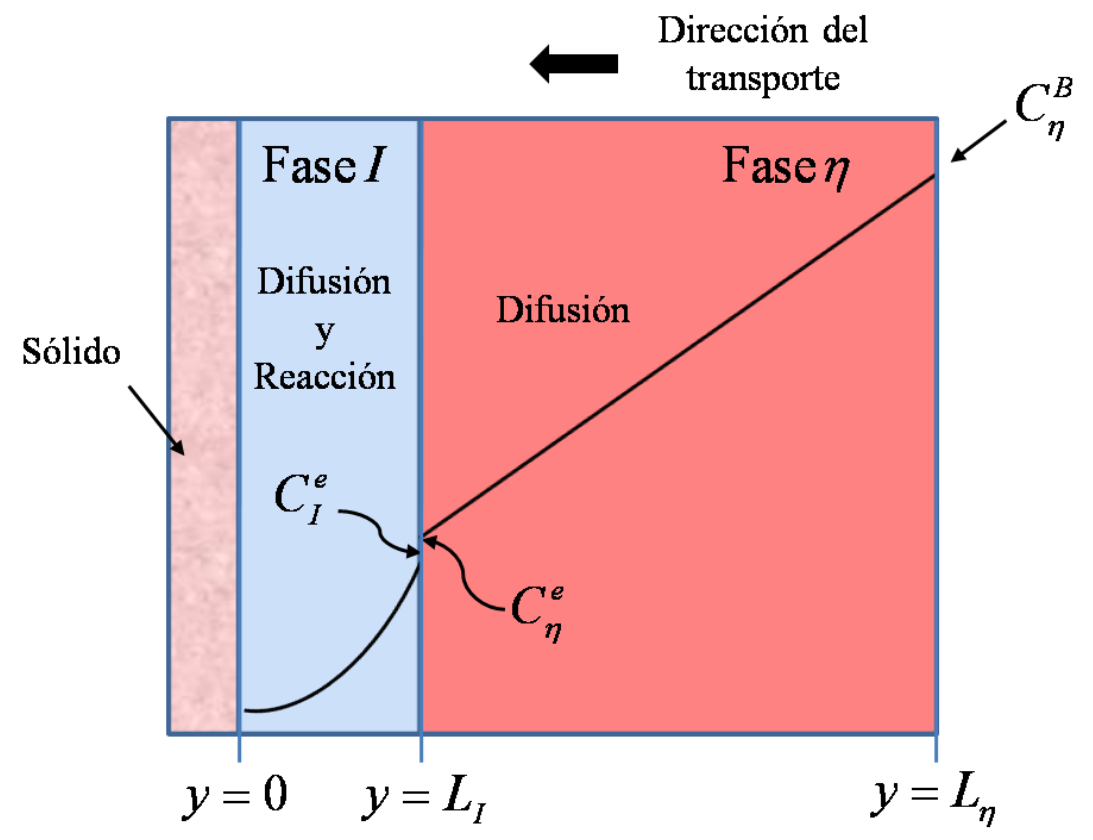

Figura 2.1 Sistema formado por dos fases con coeficientes de difusión diferentes. 
En este sistema se considera el transporte de masa unidireccional que se extiende desde $y=$ 0 hasta $y=L_{\eta}$. Se supone que la región que se extiende desde $y=0$ hasta $y=L_{I}$ esta inicialmente a la concentración $C_{I}(y)$ y tiene una difusividad $D_{I}$, mientras que la región comprendida entre $y=L_{I}$ y $y=L_{\eta}$ esta inicialmente a la concentración dada por $C_{\eta}(y)$ y tiene una difusividad $D_{\eta}$. Considerando el equilibrio que existe en la interfase entre la fase $I$ y la fase $\eta$, se puede establecer de forma esquemática las concentraciones en las fronteras y en la interfase, la cual nos ayuda a plantear las ecuaciones que rigen la transferencia de masa y sus condiciones de frontera asociadas. En la Figura 2.1 la concentración $C_{\eta}^{B}$ es la correspondiente al seno del fluido que se encuentra alrededor de la fase $\eta$. Mientras que las concentraciones en equilibrio en la interfase están representadas por $C_{I}^{e}$ y $C_{\eta}^{e}$, respectivamente.

Si se considera que $C_{\eta}^{B}$ es dependiente del tiempo, el problema consiste en evaluar los perfiles de concentración en las dos fases, así como el flux de masa en la frontera $y=L_{\eta}$, con la finalidad de encontrar bajo qué condiciones es válido el reemplazo de la ecuación diferencial gobernante de la fase $I$, por una condición de salto.

\subsection{Modelo matemático.}

La formulación matemática para el problema de transporte de masa unidimensional que gobierna la distribución de concentración en la fase $\eta$ y en la fase $I$ en estado transitorio, es el que describen las siguientes ecuaciones diferenciales parciales:

\section{Fase $\eta$}

$$
\frac{\partial C_{\eta}}{\partial t}=D_{\eta} \frac{\partial^{2} C_{\eta}}{\partial y^{2}} \quad \text { para } \quad L_{I}<y<L_{\eta}
$$


Fase I

$$
\frac{\partial C_{I}}{\partial t}=D_{I} \frac{\partial^{2} C_{I}}{\partial y^{2}}-k_{I} C_{I} \quad \text { para } \quad 0<y<L_{I}
$$

Sujeto a las condiciones de frontera

$$
\begin{gathered}
\text { En } y=L_{\eta} \quad C_{\eta}=C_{\eta}^{B}(t) \text { para } t>0 \\
\text { En } y=L_{I} \quad C_{I}^{e}=C_{\eta}^{e} K_{e q}^{\eta I} \quad \text { para } t>0 \\
\text { En } y=L_{I} \quad D_{\eta} \frac{\partial C_{\eta}^{e}}{\partial y}=D_{I} \frac{\partial C_{I}^{e}}{\partial y} \text { para } t>0 \\
\text { En } y=0 \quad \frac{\partial C_{I}}{\partial y}=0 \text { para } t>0
\end{gathered}
$$

La condición inicial está dada por:

$$
\text { Cuando } \quad t=0 \quad C_{i}=C_{i 0}(y), \quad i=\eta, I
$$

La ecuación diferencial parcial (2.1) describe una situación física en la que no existe ninguna contribución por parte del movimiento global y no existe reacción química, a diferencia de la ecuación diferencial parcial (2.2) donde ocurre una reacción lineal de primer orden. Un punto importante es que las dos ecuaciones en conjunto establecen que la velocidad de acumulación en $y=L_{\eta}$ es igual a la cantidad de masa que se difunde y reacciona a través del sistema compuesto por las dos fases.

La condición de frontera (2.3) indica que la concentración es dependiente del tiempo y la condición de frontera (2.6) expresa que el flux de masa en la pared solida es cero. Las condiciones (2.4) y (2.5) en $y=L_{I}$, describen el equilibrio entre las concentraciones de la interfase formada por las fases y la continuidad del flux, respectivamente.

Las condiciones iníciales, ecuación (2.7) se refieren específicamente a los valores de la concentración que prevalecen al iniciarse el proceso que se está estudiando. Para este caso 
las condiciones iníciales se especifican de tal manera que puede existir una distribución de concentración al iniciar la medición del tiempo representada por funciones generales que en el caso más sencillo pueden ser constantes.

El problema definido por las ecuaciones (2.1)-(2.7) y designado como modelo sin salto (MSS), puede reescribirse utilizando las variables definidas en la Tabla 2.1 en la forma adimensional siguiente:

\section{Fase $\eta$}

$$
\frac{\partial U_{\eta}}{\partial \tau}=\alpha_{\eta} \frac{\partial^{2} U_{\eta}}{\partial Y^{2}} \quad \text { para } \quad 1<Y<Y_{\eta}
$$

Fase I

$$
\frac{\partial U_{I}}{\partial \tau}=\alpha_{I} \frac{\partial^{2} U_{I}}{\partial Y^{2}}-\Phi_{I}^{2} U_{I} \quad \text { para } \quad 0<Y<1
$$

Sujeto a las condiciones de frontera

$$
\begin{gathered}
\text { En } \quad Y=Y_{\eta} \quad U_{\eta}=F_{\eta}(\tau) \text { para } \tau>0 \\
\text { En } Y=1 \quad U_{\eta}=U_{I} \text { para } \tau>0 \\
\text { En } \quad Y=1 \quad \kappa_{\eta} \frac{\partial U_{\eta}}{\partial Y}=\frac{\partial U_{I}}{\partial Y} \text { para } \tau>0 \\
\text { En } Y=0 \quad \frac{\partial U_{I}}{\partial Y}=0 \quad \text { para } \quad \tau>0
\end{gathered}
$$

La condición inicial está dada por:

$$
\text { Cuando } \quad \tau=0 \quad U_{i}=U_{i 0}(Y), \quad i=\eta, I
$$

Los detalles de la adimensionalizacion se pueden consultar en el apéndice A. 
Tabla 2.1 Definición de variables adimensionales.

\begin{tabular}{|l|l|}
\hline$Y=\frac{y}{L_{I}}$ & $\tau=\frac{t D^{*}}{L_{I}^{2}}$ \\
\hline$U_{i}=\frac{C_{i}}{C_{i}^{*}} ; \quad i=\eta, I$ \\
\hline
\end{tabular}

Tabla 2. 2 Definición de parámetros adimensionales.

\begin{tabular}{|l|l|}
\hline$\alpha_{i}=\frac{D_{i}}{D^{*}} ; \quad i=\eta, I$ & $\Phi_{I}^{2}=\frac{k_{I} L_{I}^{2}}{D^{*}}$ \\
\hline$\kappa_{\eta}=\frac{D_{\eta}}{K_{e q}^{\eta I} D_{I}}$ & $Y_{\eta}=\frac{L_{\eta}}{L_{I}}$ \\
\hline
\end{tabular}

Cabe señalar que $D^{*}$ representa el valor de una difusividad de referencia, $C_{i}^{*}$ es una concentración de referencia para cada fase, $\kappa_{\eta}$ es una constante que relaciona a la constante de equilibrio en la interfase $\left(K_{e q}^{\eta I}\right)$ con la difusividad de cada una de las fases, $Y_{\eta}$ indica la proporción entre la longitud de la fase $\eta\left(L_{\eta}\right)$ y de la fase $I\left(L_{I}\right)$ y $\Phi_{I}^{2}$ es un numero adimensional llamado Módulo de Thiele, que representa la influencia relativa de la reacción química sobre la difusión.

\subsection{Metodología para derivar la condición de salto.}

La propuesta de este trabajo para obtener la condición de salto es desarrollar un procedimiento análogo a la metodología propuesta por Ochoa-Tapia, Whitaker (1995) y Wood y col. (2000), ya que esta condición de frontera ha permitido el reemplazo de regiones de cambio abrupto de propiedades efectivas de medios porosos por condiciones de frontera para transporte de cantidad de movimiento, energía y masa. En particular para el sistema mostrado en la Figura 2.2(a), la fase $I$ seria la zona de cambio abrupto, en este caso de la concentración debido a la reacción química y considerando que la longitud característica de esta fase es menor, comparada con la longitud de la fase $\eta$, se puede aplicar los principios de dicha metodología. Por lo tanto el procedimiento a seguir para obtener la condición de salto es el siguiente, primero se aplica un operador de promedio definido por: 


$$
\{\cdot\}=\int_{0}^{1} \cdot d Y
$$

A la ecuación (2.9) de donde se obtiene

$$
\begin{gathered}
\left\{\frac{\partial U_{I}}{\partial \tau}\right\}=\left\{\alpha_{I} \frac{\partial^{2} U_{I}}{\partial Y^{2}}\right\}-\left\{\Phi_{I}^{2} U_{I}\right\} \\
\frac{\partial\left\{U_{I}\right\}}{\partial \tau}=\alpha_{I} \int_{0}^{1} \frac{\partial}{\partial Y}\left(\frac{\partial U_{I}}{\partial Y}\right) d Y-\Phi_{I}^{2}\left\{U_{I}\right\} \\
\frac{\partial\left\{U_{I}\right\}}{\partial \tau}=\left.\alpha_{I} \frac{\partial U_{I}}{\partial Y}\right|_{Y=0} ^{Y=1}-\Phi_{I}^{2}\left\{U_{I}\right\}
\end{gathered}
$$

Después se sustituye la condición de frontera (2.13) en (2.16) resultando

$$
\begin{gathered}
\frac{\partial\left\{U_{I}\right\}}{\partial \tau}=\alpha_{I}\left(\left.\frac{\partial U_{I}}{\partial Y}\right|_{Y=1}-\left.\frac{\partial U_{I}}{\partial Y}\right|_{Y=0}\right)-\Phi_{I}^{2}\left\{U_{I}\right\} \\
\frac{\partial\left\{U_{I}\right\}}{\partial \tau}=\left.\alpha_{I} \frac{\partial U_{I}}{\partial Y}\right|_{Y=1}-\Phi_{I}^{2}\left\{U_{I}\right\}
\end{gathered}
$$

Ahora se sustituye la condición de frontera dada por (2.12) en (2.17) obteniendo

$$
\frac{\partial\left\{U_{I}\right\}}{\partial \tau}=\left.\alpha_{I} \kappa_{\eta} \frac{\partial U_{\eta}}{\partial Y}\right|_{Y=1}-\Phi_{I}^{2}\left\{U_{I}\right\}
$$

Una de las ideas principales detrás del presente desarrollo es que $Y_{\eta} \gg 1$, es decir; la longitud de la fase $\eta\left(L_{\eta}\right)$ es mucho mayor a la longitud de la fase $I\left(L_{I}\right)$, por ello parece razonable reemplazar la fase $I$ de espesor 1 por una superficie divisoria en $Y=0$. Así, como consecuencia de esto y de la condición de frontera (2.11), $\left\{U_{I}\right\}$ puede ser reemplazado por $U_{\eta}$ en $Y=0$. De esta manera la ecuación (2.18) se puede expresar como una condición de salto definida como: 


$$
\frac{\partial U_{\eta}}{\partial \tau}=\left.\alpha_{I} \kappa_{\eta} \frac{\partial U_{\eta}}{\partial Y}\right|_{Y=0}-\Phi_{I}^{2} U_{\eta}
$$

El esquema resultante se muestra en la Figura 2.2(b), donde la condición de salto (2.19), reemplaza a la ecuación gobernante de la fase $I$ del sistema anterior, obteniendo un sistema que consta únicamente de la ecuación que gobierna la fase $\eta$ y una nueva condición de frontera, donde se lleva a cabo el proceso de difusión y reacción de manera simultánea. Este sistema se designa como modelo con salto (MCS), y se expresa de la siguiente forma:

\section{Fase $\eta$}

$$
\frac{\partial U_{\eta}}{\partial \tau}=\alpha_{\eta} \frac{\partial^{2} U_{\eta}}{\partial Y^{2}} \quad \text { para } \quad 0<Y<Y_{\eta}
$$

Sujeto a las condiciones de frontera

$$
\begin{gathered}
\text { En } Y=Y_{\eta} \quad U_{\eta}=F_{\eta}(\tau) \text { para } \tau>0 \\
\text { En } Y=0 \quad \frac{\partial U_{\eta}}{\partial \tau}=\left.\alpha_{I} \kappa_{\eta} \frac{\partial U_{\eta}}{\partial Y}\right|_{Y=0}-\Phi_{I}^{2} U_{\eta} \quad \text { para } \tau>0
\end{gathered}
$$

La condición inicial está dada por:

$$
\text { Cuando } \quad \tau=0 \quad U_{\eta}=U_{\eta 0}(Y)
$$




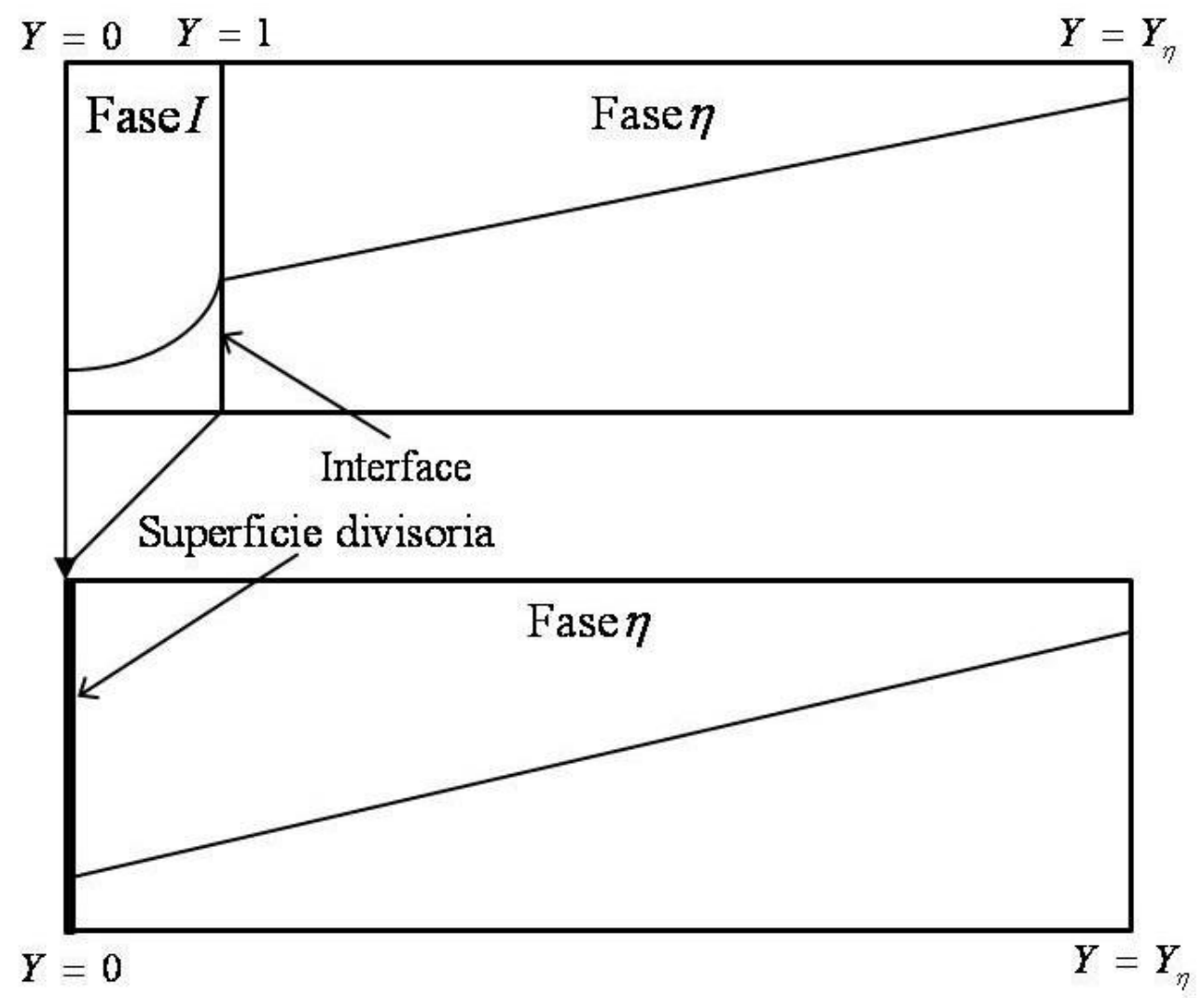

(a)

(b)

Figura 2.2 Diagrama esquemático del MSS (a) y el MCS (b), adimensional.

\subsection{Metodología de solución.}

La solución de las ecuaciones diferenciales parciales del MSS y la ecuación diferencial parcial del MCS, junto con sus respectivas condiciones de frontera y condiciones iníciales, requieren una metodología que permita, paso a paso, obtener una solución analítica. En general la metodología para resolver analíticamente los dos sistemas, está basada en la expansión en términos de las funciones propias generadas por un problema de SturmLiouville y el uso de la Formula o Identidad de Green. Los pasos principales de este método de solución son:

(a) Expandir la solución en términos de las funciones propias asociadas, lo que requiere escribir el problema de Sturm-Liouville asociado.

(b) Involucrar en la formula de Green las ecuaciones diferenciales de la solución buscada y la función propia, junto con sus condiciones de frontera. 
(c) Obtener del resultado anterior, con la propiedad de ortogonalidad de las funciones propias, los coeficientes de la expansión propuesta para la solución.

A continuación se describe a detalle cada uno de estos tres pasos.

\subsubsection{Generación y solución del problema de Sturm Liouville.}

Con el fin de obtener la solución general de los dos modelos, primero debemos encontrar la solución del problema homogéneo, para el cual se puede aplicar el método de separación de variables, proponiendo soluciones de la forma $U_{i}=\varphi_{i}(Y) G(\tau)$, y obtener el problema de Sturm-Liouville asociado. Una vez encontrada la solución del problema homogéneo, en la siguiente sección se resuelve para el problema no homogéneo. Aquí es importante reconocer que la contribución del tiempo dada por la función $G(\tau)$ es la misma para ambas fases y para los dos sistemas.

\subsubsection{Modelo sin salto.}

El problema de Sturm-Liouville para este sistema es:

$$
\frac{d^{2} \varphi_{i}}{d Y^{2}}+\mu_{i}^{2} \varphi_{i}=0 \quad\left\{\begin{array}{cc}
i=\eta, & 1<Y<Y_{\eta} \\
i=I, & 0<Y<1
\end{array}\right.
$$

Donde

$$
\mu_{I}^{2}=\alpha_{I}^{-1}\left(\lambda^{2}-\Phi_{I}^{2}\right) ; \mu_{\eta}^{2}=\alpha_{\eta}^{-1} \lambda^{2}
$$

Sujeta a las condiciones de frontera

$$
\begin{array}{lll}
\text { En } \quad Y=Y_{\eta} & \varphi_{\eta}=0 \\
\text { En } & Y=1 & \varphi_{\eta}=\varphi_{I}
\end{array}
$$




$$
\begin{gathered}
\text { En } Y=1 \quad \kappa_{\eta} \frac{d \varphi_{\eta}}{d Y}=\frac{d \varphi_{I}}{d Y} \\
\text { En } Y=0 \quad \frac{d \varphi_{I}}{d Y}=0
\end{gathered}
$$

La solución del problema de valores a la frontera definido por las ecuaciones. (2.24)-(2.29) genera las funciones propias $\varphi_{I, n}(Y), \varphi_{\eta, n}(Y)$ y la condición para encontrar los valores propios $\mu_{\eta, n}\left(\lambda_{n}\right), \mu_{I, n}\left(\lambda_{n}\right)$ :

\section{Funciones propias}

$$
\begin{gathered}
\varphi_{I, n}(Y)=\frac{\sin \left[\mu_{\eta, n}\left(1-Y_{\eta}\right)\right]}{\cos \left(\mu_{\eta, n} Y_{\eta}\right)} \frac{\cos \left(\mu_{I, n} Y\right)}{\cos \left(\mu_{I, n}\right)} \text { para } 0<Y<1 \\
\varphi_{\eta, n}(Y)=\frac{\sin \left[\mu_{\eta, n}\left(Y-Y_{\eta}\right)\right]}{\cos \left(\mu_{\eta, n} Y_{\eta}\right)} \text { para } 1<Y<Y_{\eta}
\end{gathered}
$$

Condición para los valores propios

$$
\frac{\kappa_{\eta} \mu_{\eta, n}}{\mu_{I, n}} \cos \left[\mu_{\eta, n}\left(1-Y_{\eta}\right)\right]+\frac{\sin \left(\mu_{I, n}\right)}{\cos \left(\mu_{I, n}\right)} \sin \left[\mu_{\eta, n}\left(1-Y_{\eta}\right)\right]=0
$$

Una consecuencia del problema de Sturm-Liouville es la obtención de la condición de ortogonalidad de la solución, esto es, la propiedad de otogonalidad de las funciones propias. En este caso, la condición de ortogonalidad es:

$$
\frac{\kappa_{\eta}}{\alpha_{\eta}} \int_{1}^{Y_{\eta}}\left[\varphi_{\eta, p} \varphi_{\eta, n}\right] d Y+\frac{1}{\alpha_{I}} \int_{0}^{1}\left[\varphi_{I, p} \varphi_{I, n}\right] d Y=0, \text { para } p \neq n
$$

La deducción de esta ecuación es similar a la que se obtiene cuando se resuelve el problema de Sturm-Liouville en un medio homogéneo. Sin embargo, en este caso de dos fases, es necesario involucrar a la condición interfacial. 


\subsubsection{Modelo con salto.}

El problema de Sturm-Liouville para este sistema es:

$$
\begin{gathered}
\frac{d^{2} \varphi_{\eta}^{s}}{d Y^{2}}+\left(\mu_{\eta}^{s}\right)^{2} \varphi_{\eta}^{s}=0 \quad \text { para } \quad 0<Y<Y_{\eta} \\
\text { Donde: } \mu_{\eta}^{s}=\alpha_{\eta}^{-1}\left(\lambda^{s}\right)^{2} ; \mu_{I}^{s}=\alpha_{I}^{-1}\left[\left(\lambda^{s}\right)^{2}-\Phi_{I}^{2}\right]
\end{gathered}
$$

Sujeta a las condiciones de frontera

$$
\begin{gathered}
\text { En } Y=Y_{\eta} \quad \varphi_{\eta}^{s}=0 \\
\text { En } \quad Y=0 \quad \kappa_{\eta} \varphi_{\eta}^{s}(0)+\alpha_{I}^{-1}\left[\left(\lambda^{s}\right)^{2}-\Phi_{I}^{2}\right] \varphi_{\eta}^{s}(0)=0
\end{gathered}
$$

La solución del problema de valores a la frontera definido por las ecuaciones (2.34)-(2.36) genera la función propia $\varphi_{\eta, n}^{s}$ y la condición para encontrar los valores propios $\mu_{\eta, n}^{S}\left(\lambda_{n}^{S}\right), \mu_{I, n}^{S}\left(\lambda_{n}^{S}\right):$

\section{Función propia}

$$
\varphi_{\eta, n}^{s}(Y)=\frac{\sin \left[\mu_{\eta, n}^{s}\left(Y-Y_{\eta}\right)\right]}{\cos \left(\mu_{\eta, n}^{s} Y_{\eta}\right)}
$$

Condición para los valores propios

$$
\kappa_{\eta} \mu_{\eta, n}^{s}+\left(\mu_{I, n}^{s}\right)^{2} \frac{\sin \left[-Y_{\eta} \mu_{\eta, n}^{s}\right]}{\cos \left[-Y_{\eta} \mu_{\eta, n}^{s}\right]}=0
$$

En este caso, la condición de ortogonalidad es:

$$
\frac{\kappa_{\eta}}{\alpha_{\eta}} \int_{0}^{Y_{\eta}}\left[\varphi_{\eta, p}^{s} \varphi_{\eta, n}^{s}\right] d Y+\alpha_{I}^{-1} \varphi_{\eta, p}^{s}(0) \varphi_{\eta, n}^{s}(0)=0, \quad \operatorname{para} n \neq p
$$


Todos los detalles de la solución del problema de Sturm-Liouville para los dos modelos, así como la condición de ortogonalidad asociada, se pueden revisar en el Apéndice B-1 y B-2 respectivamente.

\subsubsection{Formula de Green.}

La formula de Green está dada por la siguiente relación:

$$
\int_{a}^{b}\left[u \frac{\partial^{2} w}{\partial Y^{2}}-w \frac{\partial^{2} u}{\partial Y^{2}}\right] d Y=\left[u \frac{\partial w}{\partial Y}-w \frac{\partial u}{\partial Y}\right]_{a}^{b}
$$

Donde $w$ y $u$ son funciones de $Y$ validas en $Y \epsilon(a, b)$. Ahora se usara la formula de Green junto con la expansión de la solución buscada en términos de las funciones propias asociadas para resolver los dos modelos.

\subsubsection{Modelo sin salto.}

Para la solución de este modelo se utiliza la identidad de Green, con la finalidad de obtener las integrales para cada una de las fases que componen el sistema.

\section{Fase $\eta$}

Primero se asigna a $w=U_{\eta}$ y $u=\varphi_{\eta, p}$, después se sustituyen las ecuaciones diferenciales (2.8) y (2.24) en (2.40), resultando

$$
\begin{aligned}
\alpha_{\eta}^{-1} \int_{1}^{Y_{\eta}} \varphi_{\eta, p}\left[\frac{\partial U_{\eta}}{\partial \tau}+U_{\eta} \lambda_{p}^{2}\right] d Y= & \left.\varphi_{\eta, p}\left(Y_{\eta}\right) \frac{\partial U_{\eta}}{\partial Y}\right|_{Y=Y_{\eta}}-U_{\eta}\left(Y_{\eta}\right) \varphi_{\eta, p}^{\prime}\left(Y_{\eta}\right) \\
& -\left.\varphi_{\eta, p}(1) \frac{\partial U_{\eta}}{\partial Y}\right|_{Y=1}+U_{\eta}(1) \varphi_{\eta, p}^{\prime}(1)
\end{aligned}
$$


Ahora se usan tanto las condiciones de frontera del problema original como las del problema de Sturm-Liouville, de la fase $\eta$, para resolver el término derecho de la ecuación (2.41), obteniendo

$$
\begin{aligned}
\kappa_{\eta} \alpha_{\eta}^{-1} \int_{1}^{Y_{\eta}} \varphi_{\eta, p}\left[\frac{\partial U_{\eta}}{\partial \tau}+U_{\eta} \lambda_{p}^{2}\right] d Y= & -\kappa_{\eta} F_{\eta}(\tau) \varphi_{\eta, p}^{\prime}\left(Y_{\eta}\right) \\
& -\left.\varphi_{I, p}(1) \frac{\partial U_{I}}{\partial Y}\right|_{Y=1}+U_{I}(1) \varphi_{I, p}^{\prime}(1)
\end{aligned}
$$

Fase I

Primero se asigna a $w=U_{I}$ y $u=\varphi_{I, p}$, después se sustituyen las ecuaciones diferenciales (2.9) y (2.24) en (2.40), resultando

$$
\begin{aligned}
\alpha_{I}^{-1} \int_{0}^{1}\left[\varphi_{I, p}\left(\frac{\partial U_{I}}{\partial \tau}+\lambda_{p}^{2} U_{I}\right)\right] d Y & =\left.\varphi_{I, p}(1) \frac{\partial U_{I}}{\partial Y}\right|_{Y=1}-U_{I}(1) \varphi_{I, p}^{\prime}(1) \\
& -\left.\varphi_{I, p}(0) \frac{\partial U_{I}}{\partial Y}\right|_{Y=0}+U_{I}(0) \varphi_{I, p}^{\prime}(0)
\end{aligned}
$$

Ahora se usan tanto las condiciones de frontera del problema original como las del problema de Sturm-Liouville, de la fase $I$, para resolver el término derecho de la ecuación (2.43), obteniendo

$$
\alpha_{I}^{-1} \int_{0}^{1}\left[\varphi_{I, p}\left(\frac{\partial U_{I}}{\partial \tau}+\lambda_{p}^{2} U_{I}\right)\right] d Y=\left.\varphi_{I, p}(1) \frac{\partial U_{I}}{\partial Y}\right|_{Y=1}-U_{I}(1) \varphi_{I, p}^{\prime}(1)
$$

A continuación se suman las ecuaciones. (2.42) y (2.44) para llegar a

$$
\begin{array}{r}
\kappa_{\eta} \alpha_{\eta}^{-1} \int_{1}^{Y_{\eta}}\left[\varphi_{\eta, p}\left(\frac{\partial U_{\eta}}{\partial \tau}+\lambda_{p}^{2} U_{\eta}\right)\right] d Y+\alpha_{I}^{-1} \int_{0}^{1}\left[\varphi_{I, p}\left(\frac{\partial U_{I}}{\partial \tau}+\lambda_{p}^{2} U_{I}\right)\right] d Y= \\
-\kappa_{\eta} F_{\eta}(\tau) \varphi_{\eta, p}^{\prime}\left(Y_{\eta}\right)
\end{array}
$$


Que en forma compacta, de acuerdo a la siguiente nomenclatura $Y_{0}=0 ; Y_{1}=1 ; Y_{2}=$ $Y_{\eta} ; 1=I ; 2=\eta ; \kappa_{I}=1$, la ecuación (2.45) se puede escribirse como:

$$
\sum_{i=1}^{2} \kappa_{i} \alpha_{i}^{-1} \int_{Y_{i-1}}^{Y_{i}}\left[\varphi_{i, p}\left(\frac{\partial U_{i}}{\partial \tau}+\lambda_{p}^{2} U_{i}\right)\right] d Y=-\kappa_{\eta} F_{\eta}(\tau) \varphi_{\eta, p}^{\prime}\left(Y_{\eta}\right)
$$

Una vez que se tiene este resultado se propone la siguiente fórmula de expansión en términos de las funciones propias del problema de Sturm-Liouville asociado

$$
U_{i}(Y, \tau)=\sum_{n=1}^{\infty} \varphi_{i, n}(Y) C_{n, i}(\tau) \quad, \quad i=\eta, I
$$

Sustituyendo (2.47) en (2.46) se obtiene

$$
\sum_{i=1}^{2} \kappa_{i} \alpha_{i}^{-1} \int_{Y_{i-1}}^{Y_{i}}\left[\sum_{n=1}^{\infty} \varphi_{i, p} \varphi_{i, n}\left(\frac{\partial C_{n, i}}{\partial \tau}+\lambda_{p}^{2} C_{n, i}\right)\right] d Y=-\kappa_{\eta} F_{\eta}(\tau) \varphi_{\eta, p}^{\prime}\left(Y_{\eta}\right)
$$

La ecuación (2.48) se puede reescribir de la siguiente forma:

$$
\left[\sum_{n=1}^{\infty}\left(\frac{\partial C_{n, i}}{\partial \tau}+\lambda_{p}^{2} C_{n, i}\right)\left\{\sum_{i=1}^{2} \kappa_{i} \alpha_{i}^{-1} \int_{Y_{i-1}}^{Y_{i}} \varphi_{i, n} \varphi_{i, p} d Y\right\}\right]=-\kappa_{\eta} F_{\eta}(\tau) \varphi_{\eta, p}^{\prime}\left(Y_{\eta}\right)
$$

Al utilizar la condición de ortogonalidad dada por la ecuación (2.33), la ecuación (2.49) se reduce a

$$
\frac{\partial C_{n, i}}{\partial \tau}+\lambda_{n}^{2} C_{n, i}=\frac{I_{n}(\tau)}{D_{n}}
$$

Donde hemos usado

$$
I_{n}(\tau)=-\kappa_{\eta} F_{\eta}(\tau) \varphi_{\eta, p}^{\prime}\left(Y_{\eta}\right)
$$




$$
D_{n}=\sum_{i=1}^{2} \kappa_{i} \alpha_{i}^{-1} \int_{Y_{i-1}}^{Y_{i}} \varphi_{i, n}^{2} d Y
$$

La condición inicial para la solución de la ecuación (2.50) se obtiene de la condición inicial del problema original (2.14). Esto se muestra a continuación, primero se escribe la ecuación (2.47) a un tiempo cero, resultando:

$$
U_{i}(Y, 0)=U_{i 0}(Y)=\sum_{n=1}^{\infty} \varphi_{i, n}(Y) C_{n, i}(0)
$$

Después la ecuación se puede reescribir como:

$$
\kappa_{i} \alpha_{i}^{-1} U_{i 0}(Y) \varphi_{i, p}=\kappa_{i} \alpha_{i}^{-1} \sum_{n=1}^{\infty} \varphi_{i, p} \varphi_{i, n} C_{n, i}(0)
$$

Ahora usando la condición de ortogonalidad (2.33) en (2.54) resulta

$$
C_{n, i}(0)=\frac{\sum_{i=1}^{2} \kappa_{i} \alpha_{i}^{-1} \int_{Y_{i-1}}^{Y_{i}} U_{i 0}(Y) \varphi_{i, n}}{D_{n}}
$$

Así la solución de (2.50) está dada por

$$
C_{n, i}(\tau)=C_{n, i}(0) e^{-\lambda_{n}^{2} \tau}+\frac{1}{D_{n}} e^{-\lambda_{n}^{2} \tau} \int_{0}^{\tau} e^{\lambda_{n}^{2} \zeta} I_{n}(\zeta) d \zeta
$$

Nótese que estos coeficientes dependientes del tiempo pueden hacerse específicos de cada fase si se usa la definición de $\mu_{i, n}^{2}$, y por lo tanto la ecuación (2.56) toma la siguiente forma general, considerando que $\Phi_{\eta}^{2}=0$

$$
C_{n, i}(\tau)=C_{n, i}(0) e^{-\left(\alpha_{i} \mu_{i, n}^{2}+\Phi_{i}^{2}\right) \tau}+\frac{1}{D_{n}} e^{-\left(\alpha_{i} \mu_{i, n}^{2}+\Phi_{i}^{2}\right) \tau} \int_{0}^{\tau} e^{\left(\alpha_{i} \mu_{i, n}^{2}+\Phi_{i}^{2}\right) \zeta} I_{n}(\zeta) d \zeta
$$




\subsubsection{Modelo con salto.}

De la misma manera que en el MSS, se utiliza la identidad de Green, con la finalidad de obtener las integrales para la única fase que incluye este sistema.

\section{Fase $\eta$}

Primero se asigna a $w=U_{\eta}$ y $u=\varphi_{\eta, p}$, después se sustituyen las ecuaciones diferenciales (2.20) y (2.34) en (2.40), resultando

$$
\begin{aligned}
& \alpha_{\eta}^{-1} \int_{0}^{Y_{\eta}}\left[\varphi_{\eta, p}^{s}\left(\frac{\partial U_{\eta}}{\partial \tau}+\left(\lambda_{p}^{s}\right)^{2} U_{\eta}\right)\right] d Y=\left.\varphi_{\eta, p}^{s}\left(Y_{\eta}\right) \frac{\partial U_{\eta}}{\partial Y}\right|_{Y=Y_{\eta}}-U_{\eta}\left(Y_{\eta}\right) \varphi_{\eta}^{s}\left(Y_{\eta}\right) \\
& -\left.\varphi_{\eta, p}^{s}(0) \frac{\partial U_{\eta}}{\partial Y}\right|_{Y=0}+U_{\eta}(0) \varphi_{\eta, p}^{s}{ }^{\prime}(0)
\end{aligned}
$$

Ahora se usan tanto las condiciones de frontera del problema original como las del problema de Sturm-Liouville, de la fase $\eta$, para resolver el término derecho de la ecuación (2.58), obteniendo

$$
\begin{aligned}
\kappa_{\eta} \alpha_{\eta}^{-1} \int_{0}^{Y_{\eta}}\left[\varphi_{\eta, p}^{s}\left(\frac{\partial U_{\eta}}{\partial \tau}+\left(\lambda_{p}^{s}\right)^{2} U_{\eta}\right)\right] d Y & =-F_{\eta}(\tau) \kappa_{\eta} \varphi_{\eta, p}^{s}{ }^{\prime}\left(Y_{\eta}\right) \\
& -\left.\varphi_{\eta, p}^{s}(0) \kappa_{\eta} \frac{\partial U_{\eta}}{\partial Y}\right|_{Y=0}+U_{\eta}(0) \kappa_{\eta} \varphi_{\eta, p}^{s}{ }^{\prime}(0)
\end{aligned}
$$

De la ecuación (2.59) se puede obtener el segundo término de la derecha como sigue

$$
\begin{gathered}
\frac{\partial U_{\eta}}{\partial \tau}=\left.\alpha_{I} \kappa_{\eta} \frac{\partial U_{\eta}}{\partial Y}\right|_{Y=0}-\Phi_{I}^{2} U_{\eta} \\
\left.\kappa_{\eta} \frac{\partial U_{\eta}}{\partial Y}\right|_{Y=0}=\frac{1}{\alpha_{I}}\left(\frac{\partial U_{\eta}}{\partial \tau}+\Phi_{I}^{2} U_{\eta}\right)
\end{gathered}
$$


De igual manera el tercer término de la derecha se puede obtener de la ecuación (2.36) como sigue

$$
\begin{gathered}
\kappa_{\eta} \varphi_{\eta, n}^{s}{ }^{\prime}(0)+\alpha_{I}^{-1}\left[\left(\lambda_{n}^{s}\right)^{2}-\Phi_{I}^{2}\right] \varphi_{\eta, n}^{s}(0)=0 \\
\kappa_{\eta} \varphi_{\eta, n}^{s}{ }^{\prime}(0)=-\alpha_{I}^{-1}\left[\left(\lambda_{n}^{s}\right)-\Phi_{I}^{2}\right] \varphi_{\eta, n}^{s}(0)
\end{gathered}
$$

Sustituyendo (2.60) y (2.61) en (2.59) resulta

$$
\begin{aligned}
\kappa_{\eta} \alpha_{\eta}^{-1} \int_{0}^{Y_{\eta}}\left[\varphi_{\eta, p}^{s}\left(\frac{\partial U_{\eta}}{\partial \tau}+\lambda_{p}^{2} U_{\eta}\right)\right] d Y= & -F_{\eta}(\tau) \kappa_{\eta} \varphi_{\eta, p}^{s} \quad{ }^{\prime}\left(Y_{\eta}\right) \\
& -\varphi_{\eta, p}^{s}(0) \frac{1}{\alpha_{I}}\left(\frac{\partial U_{\eta}}{\partial \tau}+\lambda_{p}^{2} U_{\eta}\right)_{Y=0}
\end{aligned}
$$

La ecuación (2.62) se puede reescribir como

$$
\begin{array}{r}
\kappa_{\eta} \alpha_{\eta}^{-1} \int_{0}^{Y_{\eta}}\left[\varphi_{\eta, p}^{s}\left(\frac{\partial U_{\eta}}{\partial \tau}+\left(\lambda_{p}^{s}\right)^{2} U_{\eta}\right)\right] d Y+\varphi_{\eta, p}^{s}(0) \frac{1}{\alpha_{I}}\left(\frac{\partial U_{\eta}}{\partial \tau}+\lambda_{p}^{2} U_{\eta}\right)_{Y=0}= \\
-F_{\eta}(\tau) \kappa_{\eta} \varphi_{\eta, p}^{s}{ }^{\prime}\left(Y_{\eta}\right)
\end{array}
$$

Una vez que se tiene este resultado se propone la siguiente fórmula de expansión en términos de las funciones propias del problema de Sturm-Liouville asociado.

$$
U_{\eta}(Y, \tau)=\sum_{n=1}^{\infty} \varphi_{\eta, n}^{s}(Y) C_{n}^{s}(\tau)
$$

Sustituyendo (2.64) en (2.63) se obtiene

$$
\begin{aligned}
\sum_{n=1}^{\infty}\left[\frac{\partial C_{n}^{s}}{\partial \tau}+\left(\lambda_{p}^{s}\right)^{2} C_{n}^{s}\right]\left\{\kappa_{\eta} \alpha_{\eta}^{-1} \int_{0}^{Y_{\eta}}\left[\varphi_{\eta, p}^{s} \varphi_{\eta, n}^{s}\right] d Y\right. & \left.+\alpha_{I}^{-1} \varphi_{\eta, p}^{s}(0) \varphi_{\eta, n}^{s}(0)\right\}= \\
& -F_{\eta}\left(Y_{\eta}\right) \kappa_{\eta} \varphi_{\eta, p}^{s}{ }^{\prime}\left(Y_{\eta}\right)
\end{aligned}
$$


Al utilizar la condición de ortogonalidad dada por la ecuación (2.39), la ecuación (2.65) se reduce a

$$
\frac{\partial C_{n}^{s}}{\partial \tau}+\left(\lambda_{n}^{s}\right)^{2} C_{n}^{s}=\frac{I_{n}^{s}(\tau)}{D_{n}^{s}}
$$

Donde hemos usado

$$
\begin{gathered}
I_{n}^{s}(\tau)=-\kappa_{\eta} F_{\eta}(\tau) \varphi_{\eta, p}^{s}{ }^{\prime}\left(Y_{\eta}\right) \\
D_{n}^{s}=\kappa_{\eta} \alpha_{\eta}^{-1} \int_{0}^{Y_{\eta}}\left(\varphi_{\eta, n}^{s}\right)^{2} d Y+\alpha_{I}^{-1}\left(\varphi_{\eta, n}^{s}\right)^{2}(0)
\end{gathered}
$$

La condición inicial para la solución de la ecuación (2.66) se obtiene de la condición inicial del problema original (2.23). Esto se muestra a continuación, primero se escribe la ecuación (2.64) a un tiempo cero, resultando:

$$
U_{\eta}(Y, 0)=U_{\eta 0}(Y)=\sum_{n=1}^{\infty} \varphi_{\eta, n}^{s}(Y) C_{n}^{s}(0)
$$

Después la ecuación se puede reescribir como:

$$
\kappa_{\eta} \alpha_{\eta}^{-1} U_{\eta 0}(0) \varphi_{\eta, p}^{s}=\kappa_{\eta} \alpha_{\eta}^{-1} \sum_{n=1}^{\infty} \varphi_{\eta, p}^{s} \varphi_{\eta, n}^{s} C_{n}^{s}(0)
$$

Ahora usando la condición de ortogonalidad (2.39) en (2.70) resulta

$$
C_{n}^{s}(0)=\frac{\kappa_{\eta} \alpha_{\eta}^{-1} \int_{0}^{Y_{\eta}} \varphi_{\eta, n}^{s} U_{\eta 0}(Y) d Y}{\kappa_{\eta} \alpha_{\eta}^{-1} \int_{0}^{Y_{\eta}}\left(\varphi_{\eta, n}^{s}\right)^{2} d Y}
$$


Así la solución de (2.66) está dada por

$$
C_{n}^{s}(\tau)=C_{n}^{s}(0) e^{-\left(\lambda_{n}^{s}\right)^{2} \tau}+\frac{1}{D_{n}^{s}} e^{-\left(\lambda_{n}^{s}\right)^{2} \tau} \int_{0}^{\tau} e^{\left(\lambda_{n}^{s}\right)^{2} \zeta} I_{n}^{s}(\zeta) d \zeta
$$

Por último sustituyendo la ecuación (2.34) para la fase $\eta$ se obtiene

$$
C_{n}^{s}(\tau)=C_{n}^{S}(0) e^{-\left(\mu_{\eta, n}^{s}\right)^{2} \alpha_{\eta} \tau}+\frac{1}{D_{n}^{s}} e^{-\left(\mu_{\eta, n}^{s}\right)^{2} \alpha_{\eta} \tau} \int_{0}^{\tau} e^{\left(\mu_{\eta, n}^{s}\right)^{2} \alpha_{\eta} \zeta} I_{n}^{s}(\zeta) d \zeta
$$

En resumen, para evaluar el perfil de concentración dado por la ecuación (2.47) en el MSS, primero se determinan los valores propios con base a la ecuación (2.32), después se sustituyen en las ecuaciones (2.30) y (2.31) obteniendo las funciones propias para cada fase y por último se evalúa la ecuación (2.57) en función de la condición inicial $U_{i 0}(Y)$ y la funcionalidad del tiempo $F_{\eta}(\tau)$ en la frontera $Y=Y_{\eta}$. De manera similar, en el MCS, el perfil de concentración dado por la ecuación (2.64) se resuelve al sustituir los valores propios, calculados a partir de la ecuación (2.38), en la función propia de la fase $\eta$ dada por la ecuación (2.37) y después se evalúa la ecuación (2.73) en función de la condición inicial $U_{i 0}(Y)$ y la funcionalidad del tiempo $F_{\eta}(\tau)$ en la frontera $Y=Y_{\eta}$.

\subsection{Definición del flux de masa.}

Como ya se menciono anteriormente, evaluar el flux en la frontera $Y=Y_{\eta}$ tiene una gran importancia, ya que se requiere la cantidad de masa que fluye a través de esta frontera en las ecuaciones de diseño de cualquier equipo de separación donde ocurre una reacción de manera simultánea. Por lo tanto el flux de masa para concentraciones diluidas del soluto se puede calcular a partir de la siguiente relación:

$$
\left.N_{\eta}\right|_{L_{\eta}}=-D_{\eta} \frac{d C_{\eta}}{d y}
$$


Al adimensionalizar la ecuación (2.74) resulta;

$$
\left.\mathrm{N}_{\eta}\right|_{Y_{\eta}}=-\left.\alpha \frac{d U_{\eta}}{d Y}\right|_{Y_{\eta}}
$$

Donde se ha utilizado

$$
\mathrm{N}_{\eta}=\frac{N_{\eta}}{N_{\eta}^{*}}, N_{\eta}^{*}=D^{*} C_{\eta}^{*} L_{I}^{-1}
$$

En este punto se cuenta con una solución analítica para los dos modelos y la expresión para evaluar el flux de masa en la frontera de interés. 


\section{CAPÍTULO 3}

\section{CASOS DE ESTUDIO}

Con el fin de evaluar las soluciones analíticas de los dos modelos, es necesario resolver las integrales indicadas para diferentes casos donde la concentración dependa del tiempo. En esta sección se propone dos diferentes tipos de funcionalidades para el problema lineal (Caso I-a y I-b) y se desarrollan las soluciones correspondientes. Además con la finalidad de ampliar el análisis de diversos problemas de aplicación, se propone estudiar dos problemas no lineales, el primero considera en la fase $I$ una reacción de segundo orden (Caso II) y el segundo problema una cinética de Michaelis Menten (Caso III). Está claro que la solución de estos problemas será de forma numérica, pero siguiendo la misma metodología que se utilizo en el caso lineal.

Antes de explicar cada uno de los problemas de estudio, se establece que para todos los casos de análisis, al inicio del proceso la concentración del soluto $A$ en las dos fases es cero; es decir, la condición inicial para los dos sistemas es:

MSS

$$
\tau=0 \quad U_{i}=U_{i 0}(Y)=0, \quad i=\eta, I
$$

MCS

$$
\tau=0 \quad U_{\eta}=U_{\eta 0}(Y)=0
$$




\subsection{Caso I. Reacción lineal en la fase $I$.}

Con base en lo anterior, en la solución analítica el término $C_{n}(0)$ para los dos modelos es cero, por lo tanto las funciones que dependen del tiempo $C_{n}(\tau)$ se reducen a un solo término como se muestra en la Tabla 3.1. Además en la misma tabla se indican las expresiones para la constante $D_{n}$ en los dos sistemas, que se requieren para completar las soluciones.

Tabla 3.1 Coeficientes $C_{n, i}(\tau)$ y $D_{n}$ para el MSS y $C_{\eta}^{s}(\tau)$ y $D_{n}^{s}$ para el MCS.

\begin{tabular}{|c|}
\hline MSS \\
\hline$C_{n, \eta}(\tau)=\frac{1}{D_{n}} e^{-\left(\alpha_{\eta} \mu_{\eta, n}^{2}\right) \tau} \int_{0}^{\tau} e^{\left(\alpha_{\eta} \mu_{\eta, n}^{2}\right) \zeta} I_{n}(\zeta) d \zeta$ \\
\hline Fase $I$ \\
\hline$C_{n, I}(\tau)=\frac{1}{D_{n}} e^{-\left(\alpha_{I} \mu_{I, n}^{2}+\Phi_{I}^{2}\right) \tau} \int_{0}^{\tau} e^{\left(\alpha_{I} \mu_{I, n}^{2}+\Phi_{I}^{2}\right) \zeta} I_{n}(\zeta) d \zeta$ \\
\hline$D_{n}=\frac{\sin ^{2}\left[\mu_{\eta, n}\left(1-Y_{\eta}\right)\right]}{\cos ^{2}\left(\mu_{\eta, n} Y_{\eta}\right) \cos ^{2}\left(\mu_{I, n}\right)}\left[\frac{2 \mu_{I, n}+\operatorname{sen}\left(2 \mu_{I, n}\right)}{4 \mu_{I, n} \alpha_{I}}\right]+\left(\frac{\kappa_{\eta}}{\alpha_{\eta}}\right) \frac{\operatorname{sen}\left[2 \mu_{\eta, n}\left(1-Y_{\eta}\right)\right]-2 \mu_{\eta, n}\left(1-Y_{\eta}\right)}{4 \mu_{\eta, n} \cos ^{2}\left(\mu_{\eta, n} Y_{\eta}\right)}$ \\
\hline$C_{n}^{s}(\tau)=\frac{1}{D_{n}^{s}} e^{-\left(\mu_{n}^{s}\right)^{2} \alpha_{\eta} \tau} \int_{0}^{\tau} e^{\left(\mu_{n}^{s}\right)^{2} \alpha_{\eta} \zeta} I_{n}^{s}(\zeta) d \zeta$ \\
\hline$D_{n}^{s}=\frac{\kappa_{\eta}}{\alpha_{\eta} \cos ^{2}\left(\mu_{\eta, n}^{s} Y_{\eta}\right)}\left[\frac{\operatorname{sen}\left[-2 \mu_{\eta, n}^{s} Y_{\eta}\right]+2 \mu_{\eta, n}^{s} Y_{\eta}}{4 \mu_{\eta, n}^{s}}\right]+\frac{\sin ^{2}\left[-\mu_{\eta, n}^{s} Y_{\eta}\right]}{\alpha_{I} \cos ^{2}\left(\mu_{\eta, n}^{s} Y_{\eta}\right)}$ \\
\hline
\end{tabular}

Para poder realizar una evaluación de estas soluciones debemos proponer funcionalidades para las concentraciones en la frontera $Y=Y_{\eta}$, en este trabajo analizaremos dos funcionalidades del tiempo diferentes para estas concentraciones adimensionales, con el fin de evaluar los términos $C_{n, \eta}(\tau), C_{n, I}(\tau)$ y $C_{n}^{s}(\tau)$. 
Los detalles correspondientes a la evaluación de los términos $D_{n}$ y $D_{n}^{S}$ se encuentran disponibles en el Apéndice B-3.

\subsubsection{Caso I-a. La concentración en el seno del fluido externo a la fase $\boldsymbol{\eta}$ es constante.}

Para los dos modelos la concentración en la frontera $Y=Y_{\eta}$ es una constante, de manera que

$$
\text { En } \quad Y=Y_{\eta} \quad U_{\eta}=F_{\eta}(\tau)=U_{\eta}^{1}
$$

Por lo tanto los términos $C_{n, i}(\tau)$ para el MSS son:

Fase $\eta$

$$
C_{n, \eta}(\tau)=\frac{1}{D_{n}} \frac{\kappa_{\eta} U_{\eta}^{1}}{\cos \left(\mu_{\eta, n} Y_{\eta}\right) \alpha_{\eta} \mu_{\eta, n}}\left[e^{-\left(\alpha_{\eta} \mu_{\eta, n}^{2}\right) \tau}-1\right]
$$

Fase I

$$
C_{n, I}(\tau)=\frac{1}{D_{n}} \frac{\kappa_{\eta} U_{\eta}^{1} \mu_{\eta, n}}{\cos \left(\mu_{\eta, n} Y_{\eta}\right)} \frac{1}{\left(\alpha_{I} \mu_{I, n}^{2}+\Phi_{I}^{2}\right)}\left[e^{-\left(\alpha_{I} \mu_{I, n}^{2}+\Phi_{I}^{2}\right) \tau}-1\right]
$$

De manera similar el término $C_{n}^{S}(\tau)$ para el MCS es:

$$
C_{n}^{s}(\tau)=\frac{1}{D_{n}^{s}} \frac{\kappa_{\eta} U_{\eta}^{1}}{\cos \left(\mu_{\eta, n}^{s} Y_{\eta}\right) \alpha_{\eta} \mu_{\eta, n}^{s}}\left[e^{-\left(\alpha_{\eta}\left(\mu_{\eta, n}^{s}\right)^{2}\right) \tau}-1\right]
$$




\subsubsection{Caso I-b. La concentración en el seno del fluido externo a la fase $\eta$ depende} exponencialmente del tiempo.

Para los dos modelos la concentración en la frontera $Y=Y_{\eta}$, depende exponencialmente del tiempo, por lo tanto

$$
\text { En } \quad Y=Y_{\eta} \quad U_{\eta}=F_{\eta}(\tau)=U_{\eta}^{1}-\sigma_{\eta} e^{-\beta_{\eta} \tau}
$$

Donde $\sigma_{\eta}$ es un parámetro pre-exponencial y $\beta_{\eta}$ es un factor de decaimiento. Por lo tanto los términos $C_{n, i}(\tau)$ para el MSS son:

Fase $\eta$

$$
C_{n, \eta}(\tau)=\frac{R_{\eta} U_{\eta}^{1}}{\left(\alpha_{\eta} \mu_{\eta, n}^{2}\right)}\left[1-e^{-\left(\alpha_{\eta} \mu_{\eta, n}^{2}\right) \tau}\right]-\frac{R_{\eta} \sigma_{\eta}}{\left(\alpha_{\eta} \mu_{\eta, n}^{2}-\beta_{\eta}\right)}\left[e^{-\beta_{\eta} \tau}-e^{-\left(\alpha_{\eta} \mu_{\eta, n}^{2}\right) \tau}\right]
$$

Fase I

$$
\begin{aligned}
C_{n, I}(\tau)=\frac{R_{\eta} U_{\eta}^{1}}{\left(\alpha_{I} \mu_{I, n}^{2}+\Phi_{I}^{2}\right)}\left[1-e^{-\left(\alpha_{I} \mu_{I, n}^{2}+\Phi_{I}^{2}\right) \tau}\right] & \\
& -\frac{R_{\eta} \sigma_{\eta}}{\left(\alpha_{I} \mu_{I, n}^{2}+\Phi_{I}^{2}-\beta_{\eta}\right)}\left[e^{-\beta_{\eta} \tau}-e^{-\left(\alpha_{I} \mu_{I, n}^{2}+\Phi_{I}^{2}\right) \tau}\right]
\end{aligned}
$$

Donde

$$
R_{\eta}=-\frac{\kappa_{\eta} \mu_{\eta, n}}{D_{n} \cos \left(\mu_{\eta, n} Y_{\eta}\right)}
$$

De manera similar el término $C_{n}^{S}(\tau)$ para el MCS es: 


$$
C_{n}^{s}(\tau)=\frac{\kappa_{\eta} \mu_{\eta, n}^{s}}{D_{n}^{s} \cos \left(\mu_{\eta, n}^{s} Y_{\eta}\right)}\left[\begin{array}{l}
\frac{U_{\eta}^{1}}{\left(\mu_{\eta, n}^{s}\right)^{2} \alpha_{\eta}}\left(e^{-\left(\mu_{\eta, n}^{s}\right)^{2} \alpha_{\eta} \tau}-1\right) \\
+\frac{\sigma_{\eta}}{\left[\left(\mu_{\eta, n}^{s}\right)^{2} \alpha_{\eta}-\beta_{\eta}\right]}\left(e^{-\beta_{\eta} \tau}-e^{-\left(\mu_{\eta, n}^{s}\right)^{2} \alpha_{\eta} \tau}\right)
\end{array}\right]
$$

Todos los detalles para la obtención de los términos anteriores se pueden consultar en el Apéndice B-4.

\subsection{Caso II. Reacción no lineal de segundo orden.}

La ecuación de velocidad de reacción esta dado por:

$$
-r_{I}=K_{I} C_{I}^{2}
$$

Por lo tanto la formulación matemática para el problema unidimensional de transporte de masa que gobierna la distribución de concentración en la fase $\eta$ y en la fase $I$ en estado transitorio, es el que describen las siguientes ecuaciones diferenciales parciales adimensionales:

Fase $\eta$

$$
\frac{\partial U_{\eta}}{\partial \tau}=\alpha_{\eta} \frac{\partial^{2} U_{\eta}}{\partial Y^{2}} \quad \text { para } \quad 1<Y<Y_{\eta}
$$

Fase I

$$
\frac{\partial U_{I}}{\partial \tau}=\alpha_{I} \frac{\partial^{2} U_{I}}{\partial Y^{2}}-\Phi_{I}^{2} U_{I}^{2} \quad \text { para } \quad 0<Y<1
$$

Donde el termino del módulo de Thiele sufre una modificación, de tal manera que 


$$
\Phi_{I}^{2}=\frac{k_{I} L^{2}}{D^{*}} ; k_{I}=C_{I}^{*} K_{I}
$$

Las condiciones de frontera y la condición inicial, son similares a las utilizadas en el problema lineal. Siguiendo los pasos de la metodología para obtener la condición de salto, el modelo matemático resultante para este sistema se expresa de la siguiente manera:

Fase $\eta$

$$
\frac{\partial U_{\eta}}{\partial \tau}=\alpha_{\eta} \frac{\partial^{2} U_{\eta}}{\partial Y^{2}} \quad \text { para } \quad 0<Y<Y_{\eta}
$$

Sujeto a las condiciones de frontera

$$
\begin{gathered}
\text { En } Y=Y_{\eta} \quad U_{\eta}=F_{\eta}(\tau) \text { para } \tau>0 \\
\text { En } Y=0 \quad \frac{\partial U_{\eta}}{\partial \tau}=\left.\alpha_{I} \kappa_{\eta} \frac{\partial U_{\eta}}{\partial Y}\right|_{Y=1}-\Phi_{I}^{2} U_{\eta}^{2} \quad \text { para } \tau>0
\end{gathered}
$$

La condición inicial está dada por:

$$
\text { Cuando } \quad \tau=0 \quad U_{\eta}=U_{\eta 0}(Y)
$$

\subsection{Caso III. Cinética de Michaelis Menten.}

Ahora el término no lineal se reemplaza por una reacción enzimática, para ello se utiliza la ecuación de Michaelis Menten como una expresión de velocidad para esta reacción, la cual está dada por:

$$
-r_{I}=v_{0}=\frac{V_{\max } C_{I}}{K_{M}+C_{I}}
$$

Donde $V_{\max }$ es la velocidad máxima de una reacción que se produce cuando la enzima se satura, debido a elevadas concentraciones de sustrato $C_{I}$. Esta velocidad se calcula con la concentración total de la enzima y la constante de velocidad $k_{2}$, obteniendo $V_{\max }=k_{2}[E]_{T}$ 
y $K_{M}$ se define como la concentración del sustrato cuando $v_{0}$ es igual a la mitad de la $V_{\max }$ y se conoce como la constante de Michaelis. A partir de las definiciones de las variables y parámetros adimensionales de las Tablas 2.1 y 2.2, se puede derivar una expresión adimensional para la ecuación (3.25), obteniendo,

$$
V_{0}=\frac{\Phi_{M}^{2} U_{I}}{\kappa_{M}+U_{I}}
$$

Donde

$$
V_{0}=\frac{L_{I}^{2} V_{0}}{D^{*}} ; \quad \Phi_{M}^{2}=\frac{L_{I}^{2} V_{\max }}{D^{*} C_{I}^{*}} ; \quad \kappa_{M}=\frac{K_{M}}{C_{I}^{*}}
$$

Para nuestro sistema, la concentración adimensional en la fase $I\left(U_{I}\right)$ tiene un rango de $0 \leq$ $U_{I} \leq 1$. Con base en lo anterior y a la ecuación (3.26) se puede describir una curva, como se muestra en la Figura 3.1, donde se puede notar que cuando $U_{I} \ll \kappa_{M}$ :

$$
V_{0} \approx \frac{\Phi_{M}^{2} U_{I}}{\kappa_{M}}
$$

La cual es una cinética de primer orden, que se puede apreciar en la parte creciente de la Figura 3.1. Por otro lado, si $U_{I} \gg \kappa_{M}$

$$
V_{0} \approx \Phi_{M}^{2}
$$

En este caso la cinética es de orden cero y se identifica con la parte aproximadamente horizontal de la Figura 3.1. Como se puede notar, esta expresión tiene dos extremos, sin embargo, en este trabajo se considerara la forma completa, es decir, la ecuación (3.26) la cual es no lineal. 


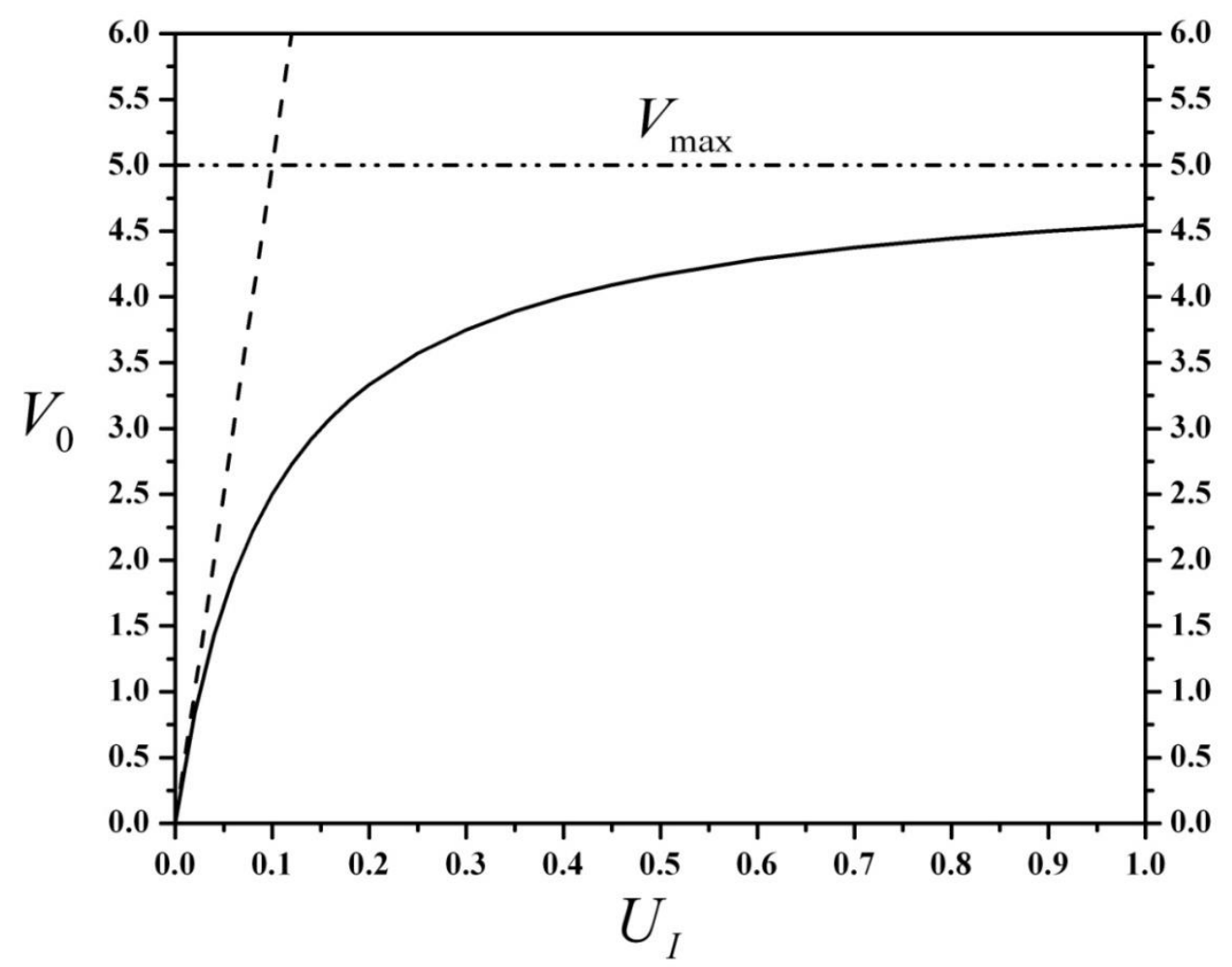

Figura 3.1 Grafica de $V_{0}$ en función de $U_{I}$ para una reacción catalizada por una enzima.

Por lo tanto la formulación matemática para el problema unidimensional de transporte de masa que gobierna la distribución de concentración en la fase $\eta$ y en la fase $I$ en estado transitorio, es el que describen las siguientes ecuaciones diferenciales parciales adimensionales:

Fase $\eta$

$$
\frac{\partial U_{\eta}}{\partial \tau}=\alpha_{\eta} \frac{\partial^{2} U_{\eta}}{\partial Y^{2}} \quad \text { para } \quad 1<Y<Y_{\eta}
$$

Fase I

$$
\frac{\partial U_{I}}{\partial \tau}=\alpha_{\mathrm{I}} \frac{\partial^{2} U_{I}}{\partial Y^{2}}-\frac{\Phi_{M}^{2} U_{I}}{\left(\kappa_{M}+U_{I}\right)} \quad \text { para } \quad 0<Y<1
$$


Donde el término del módulo de Thiele sufre una modificación ya definido en la ecuación (3.27). Las condiciones de frontera y la condición inicial, son similares a las utilizadas en el problema lineal. Siguiendo los pasos de la metodología para obtener la condición de salto, el modelo matemático resultante para este sistema se expresa de la siguiente manera:

Fase $\eta$

$$
\frac{\partial U_{\eta}}{\partial \tau}=\alpha_{\eta} \frac{\partial^{2} U_{\eta}}{\partial Y^{2}} \quad \text { para } \quad 0<Y<Y_{\eta}
$$

Sujeto a las condiciones de frontera

$$
\begin{gathered}
\text { En } \quad Y=Y_{\eta} \quad U_{\eta}=F_{\eta}(\tau) \text { para } \tau>0 \\
\text { En } Y=0 \quad \frac{\partial U_{\eta}}{\partial \tau}=\left.\alpha_{I} \kappa_{\eta} \frac{\partial U_{\eta}}{\partial Y}\right|_{Y=1}-\Phi_{M}^{2}\left(\frac{U_{\eta}}{\kappa_{M}+U_{\eta}}\right) \text { para } \tau>0
\end{gathered}
$$

La condición inicial está dada por:

$$
\text { Cuando } \quad \tau=0 \quad U_{\eta}=U_{\eta 0}(Y)
$$




\section{CAPÍTULO 4}

\section{RESULTADOS Y DISCUSIÓN}

El objetivo de evaluar la solución analítica del problema lineal es obtener el error del flux en la frontera $Y=Y_{\eta}$ entre los dos modelos, el MSS y el MCS, con la finalidad de encontrar bajo qué condiciones es válido el reemplazo de la ecuación diferencial gobernante de la fase $I$, por una condición de salto. A continuación, para cada uno de los casos de estudio, se evalúa la solución analítica para el problema lineal. Además de manera similar se analizan los problemas no lineales, utilizando el algoritmo de Newton Raphson multivariable para evaluar el estado estacionario y el método de líneas para el estado transitorio.

En general los valores de los parámetros utilizados en la evaluación de las soluciones de los diferentes casos, que aquí se reportan, son:

(a) El módulo de Thiele $\Phi_{I}^{2}$ varía en un rango de 0.1-10.0. Estos valores se seleccionaron de tal manera que se pueda apreciar el efecto de la reacción química en la fase $I$; es decir, a valores pequeños de $\Phi_{I}^{2}$, el transporte por difusión en la fase I predomina. Por otro lado el efecto de la reacción sobre el transporte del soluto es mayor a valores altos de este número adimensional.

(b) La longitud de la fase $I$ se mantiene constante a un valor de $L_{I}=1$, de tal manera que los valores que se utilicen para $Y_{\eta}$ en la evaluación de los perfiles, sean directamente los valores de $L_{\eta}$. 
(c) $\alpha_{\eta}=3.0$ y $\alpha_{\mathrm{I}}=1.0$. Estos valores indican que la difusividad del soluto en la fase $I$ $\left(D_{I}\right)$, es igual a la difusividad de referencia, $\left(D^{*}\right)$ mientras tanto la difusividad en la fase $\eta\left(D_{\eta}\right)$ es mayor a la de referencia para el caso donde la fase $\eta$ sea un gas.

(d) $\kappa_{\eta}=2.0$, Lo cual implica que la constante de equilibrio en la interfase entre la fase $\eta$ y la fase $I$ es $K_{e q}^{\eta I}=1.5$, esto indica que el transporte se favorece hacia la fase $I$.

(e) Por último, el parámetro pre-exponencial $\sigma_{\eta}=1.0$, el factor de decaimiento de la función exponencial $\beta_{\eta}=10.0$, fueron seleccionados tales que representen cambios suaves de las concentraciones en la frontera $Y=Y_{\eta}$ para el Caso I-b.

A manera de resumen y con base a lo anterior, en la tabla 4.1 se muestra los parámetros utilizados para la evaluación de cada caso.

Tabla 4.1 Valores de los parámetros adimensionales utilizados en las soluciones de los diferentes casos.

\begin{tabular}{|c|c|c|c|c|c|c|c|c|c|c|}
\hline Caso & $\alpha_{\eta}$ & $\alpha_{I}$ & $\kappa_{\eta}$ & $U_{\eta}^{1}$ & $\sigma_{\eta}$ & $\beta_{\eta}$ & $\kappa_{M}$ & $\Phi_{I}^{2}$ & $\Phi_{M}^{2}$ & $Y_{\eta}$ \\
\hline I-a & $\mathbf{3 . 0}$ & $\mathbf{1 . 0}$ & $\mathbf{2 . 0}$ & $\mathbf{1 . 0}$ & $\times$ & $\times$ & $\times$ & $\mathbf{0 . 1 - 1 0 . 0}$ & $\times$ & $\mathbf{2 . 0 - 6 0 . 0}$ \\
\hline I-b & $\mathbf{3 . 0}$ & $\mathbf{1 . 0}$ & $\mathbf{2 . 0}$ & $\mathbf{1 . 0}$ & $\mathbf{1 . 0}$ & $\mathbf{1 0 . 0}$ & $\times$ & $\mathbf{0 . 1 - 1 0 . 0}$ & $\times$ & $\mathbf{2 . 0 - 6 0 . 0}$ \\
\hline II & $\mathbf{3 . 0}$ & $\mathbf{1 . 0}$ & $\mathbf{2 . 0}$ & $\mathbf{1 . 0}$ & $\times$ & $\times$ & $\times$ & $\mathbf{0 . 1 - 1 0 . 0}$ & $\times$ & $\mathbf{2 . 0 - 4 5 . 0}$ \\
\hline III & $\mathbf{3 . 0}$ & $\mathbf{1 . 0}$ & $\mathbf{2 . 0}$ & $\mathbf{1 . 0}$ & $\times$ & $\times$ & $\mathbf{0 . 1}$ & $\times$ & $\mathbf{0 . 5 - 1 0 . 0}$ & $\mathbf{2 . 0 - 9 0 . 0}$ \\
\hline
\end{tabular}

Antes de exponer los resultados, en el apéndice $\mathrm{C}$ se presenta los diagramas de flujo utilizados para evaluar la solución analítica para el caso I-a y I-b. Además de los algoritmos de los métodos numéricos empleados para los casos II y III.

\subsection{Caso I-a. La concentración en el seno del fluido externo a la fase $\boldsymbol{\eta}$ es constante.}

La concentración adimensional en la frontera $Y=Y_{\eta}$ se considera constante y es igual a uno. En la Figura 4.1 se muestra las curvas del $\%$ error del flux de masa en $Y=Y_{\eta}$ en estado estacionario en función del $\Phi_{I}^{2}$ y $Y_{\eta}$, comparando el flux del MSS y MCS, calculados a partir de la ecuación (2.75). La expresión utilizada para evaluar el \% Error está definida como: 


$$
\% \text { Error }=\left(\frac{\left.N_{\eta}\right|_{Y=Y_{\eta}} M S S-\left.N_{\eta}\right|_{Y=Y_{\eta}} M C S}{\left.N_{\eta}\right|_{Y=Y_{\eta}} M S S}\right) \times 100
$$

Como se observa en la Figura 4.1 conforme aumenta el valor de $Y_{\eta}$, los valores del $\%$ de error disminuyen a cualquier valor del módulo de Thiele. Sin embargo, el efecto de la reacción en la fase $I$ sobre todo el sistema es importante, ya que a valores de $0.5<\Phi_{\mathrm{I}}^{2}<2$ y $1.1<Y_{\eta}<9$ se localiza una zona donde los valores del \% Error varían en un rango de 19.4-2.7. Por otro lado en la sección limitada por $28<Y_{\eta}<54$ y a $2<\Phi_{\mathrm{I}}^{2}<10$, se encuentran valores del \% Error en un rango de 2-0.7, en esta sección se observa que manteniendo un \% Error de 1.0 se requiere un valor de $Y_{\eta}=48$ a un $\Phi_{\mathrm{I}}^{2}=5$, es decir; cuando en la fase $I$ el coeficiente de difusión es 5 veces mayor a la constante de velocidad de reacción por su longitud al cuadrado, los valores del flux de masa para el MSS y MCS serán de $\left.N_{\eta}\right|_{Y=Y_{\eta}}=0.06262$ y $\left.N_{\eta}\right|_{Y=Y_{\eta}}=0.062$, respectivamente, solamente si la longitud de la fase $\eta$ es 48 veces mayor a la longitud de la fase $I$. De manera similar para otros valores de $\Phi_{\mathrm{I}}^{2}=0.1$ y $\Phi_{\mathrm{I}}^{2}=10$ se requieren valores de $Y_{\eta}=14$ y $Y_{\eta}=56$, respectivamente como se muestra en la Figura 4.2.

En la Figura 4.3 se muestran los resultados de la evolución del flux de masa en $Y=Y_{\eta}$ evaluados con los dos modelos para un valor de $Y_{\eta}=5$, el \% Error del flux a un tiempo de $1 \times 10^{-5}$ es cero para este sistema, pero a medida que aumenta el tiempo y alcanzando el estado estacionario el \%Error $=8.97$. Por otro lado para un valor de $Y_{\eta}=48$, el \% Error del flux de masa de los dos modelos desde un tiempo cero hasta alcanzar el estado estacionario a un $\tau>900$, se mantiene por debajo de 0.99 como se muestra en la Figura 4.4. En ambos sistemas se mantiene constante el valor de $\Phi_{\mathrm{I}}^{2}=5$.Para obtener los resultados de la Figura 4.4 se utilizaron, 270 y 266 valores propios de la serie para tiempos cortos en el MSS y MCS respectivamente y solo un valor propio en los dos modelos para alcanzar el estado estacionario. La búsqueda de los valores propios se explica en el apéndice D. 


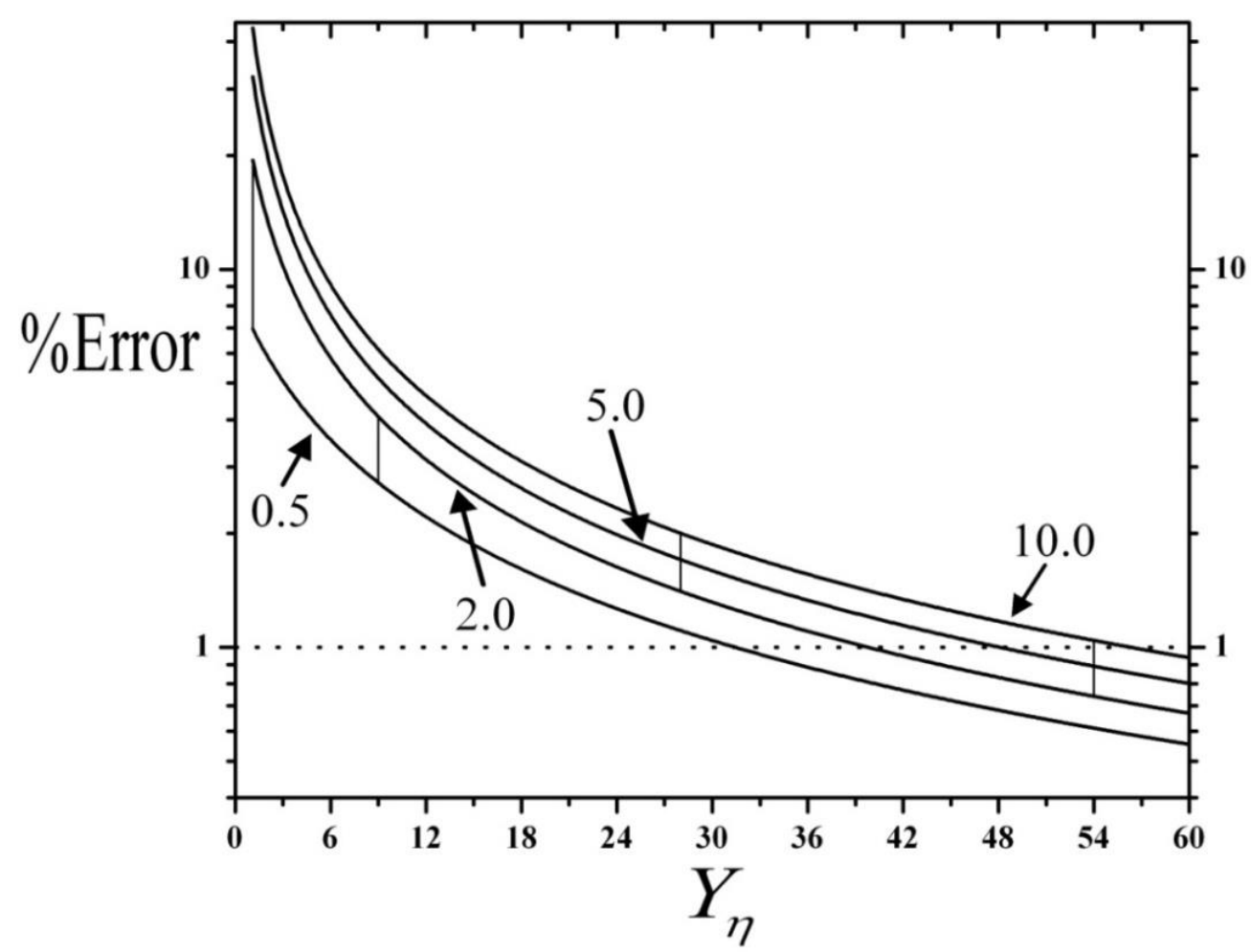

Figura 4.1 \% Error en función de $Y_{\eta}$ a diferentes valores de $\Phi_{I}^{2}$. Caso I-a.

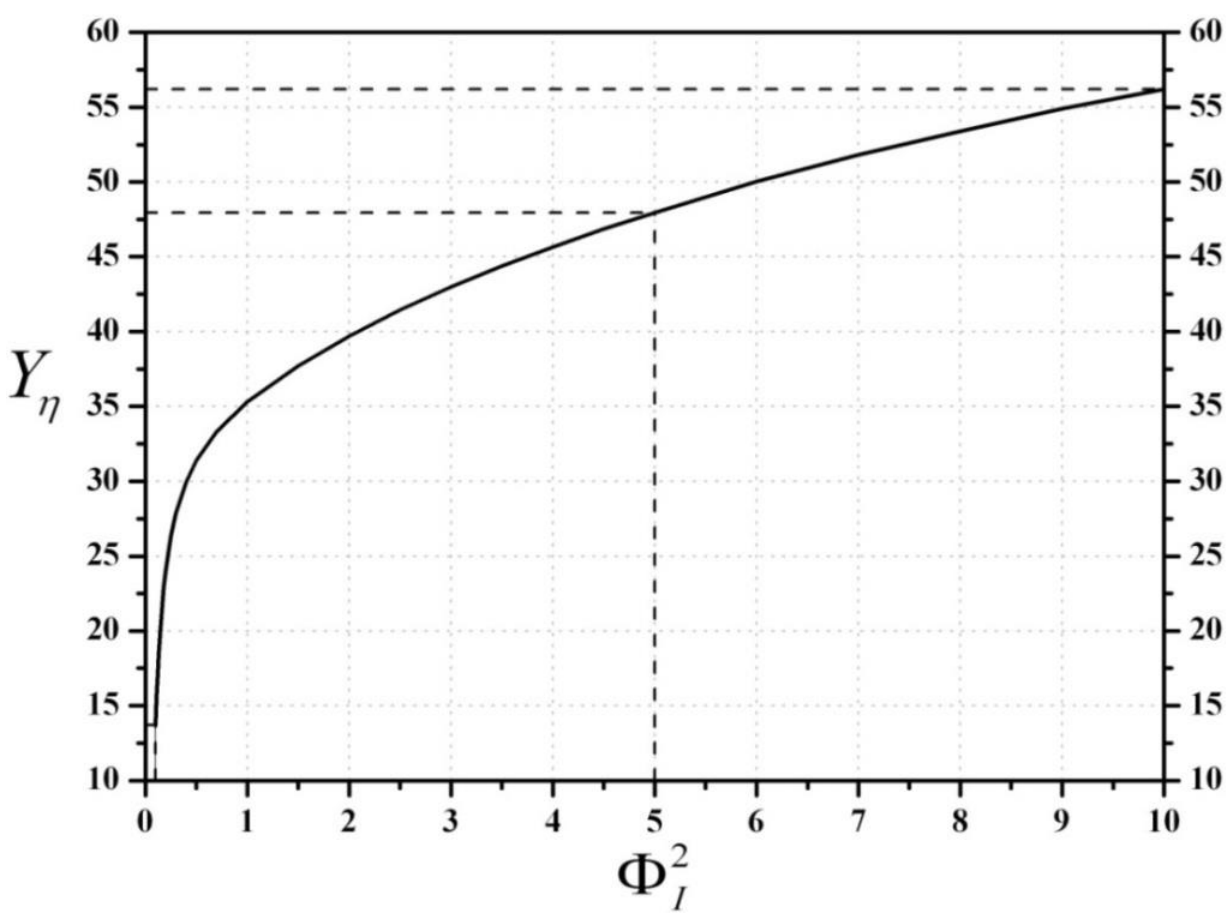

Figura 4.2 $Y_{\eta}$ requerida para mantener un $\%$ Error de 1.0, en función de $\Phi_{I}^{2}$. Caso I-a. 
Cabe señalar que al evaluar el flux de masa en $Y=Y_{\eta}$ con la solución analítica en estado transitorio que fue obtenida mediante la Formula de Green, se presenta un problema relacionado con este método de solución llamado fenómeno de Gibbs (Fay y Kloppers, 2001), ya que al utilizar series de Fourier en la metodología, los valores de concentración adimensional convergen a cero conforme se aproximan a esta frontera. Para solucionar el problema anterior se utilizo el método de superposición con la intención de combinar la solución en estado estacionario y la solución del estado transitorio. Los detalles correspondientes para la evaluación del flux de masa mediante el método de superposición para los dos modelos se encuentran disponibles en el Apéndice E.

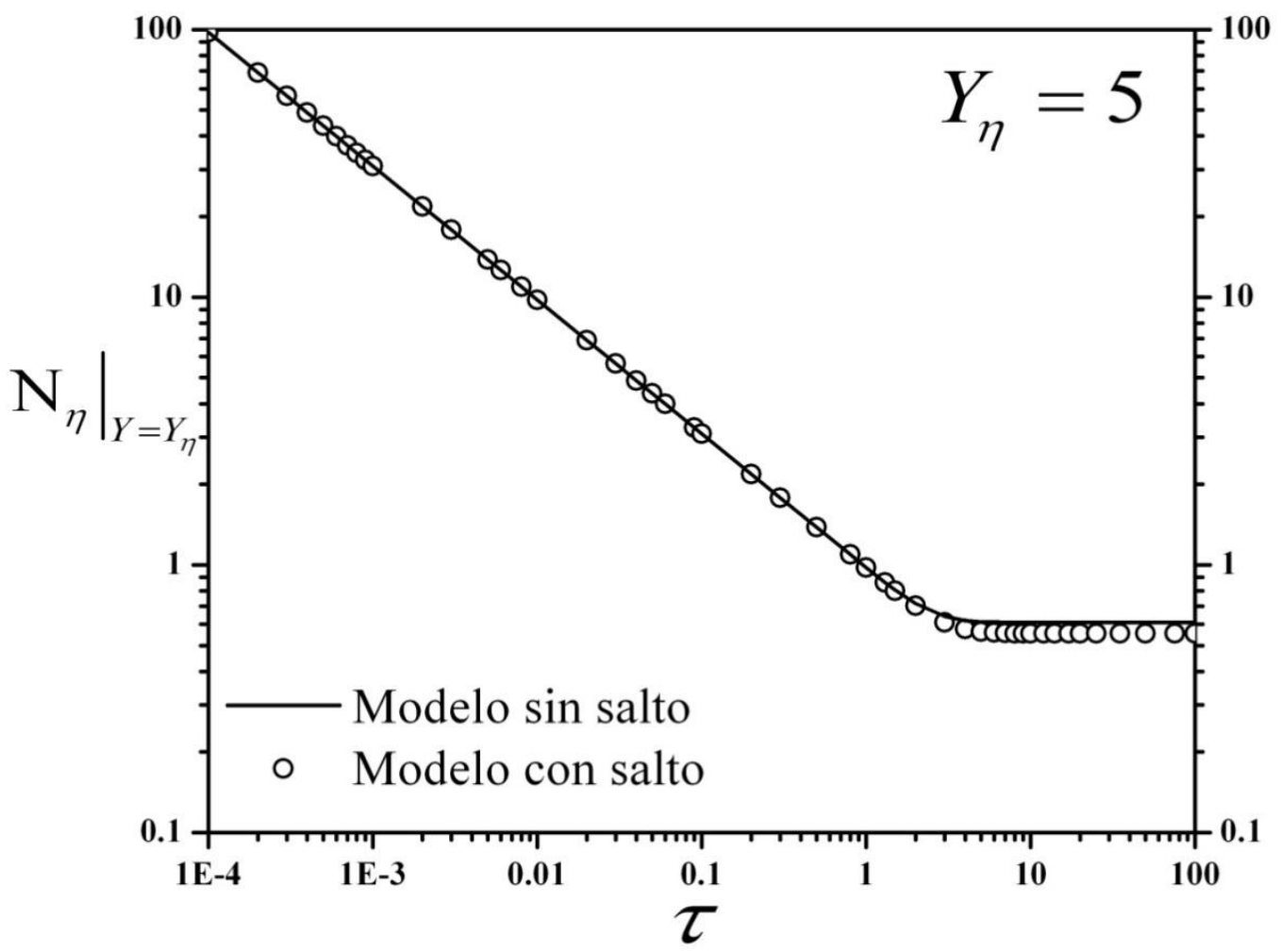

Figura 4.3 Evolución del flux de masa evaluado en $Y=Y_{\eta}$ para el Caso I-a, manteniendo constante un $\Phi_{\mathrm{I}}^{2}=5.0$ y $Y_{\eta}=5$. 


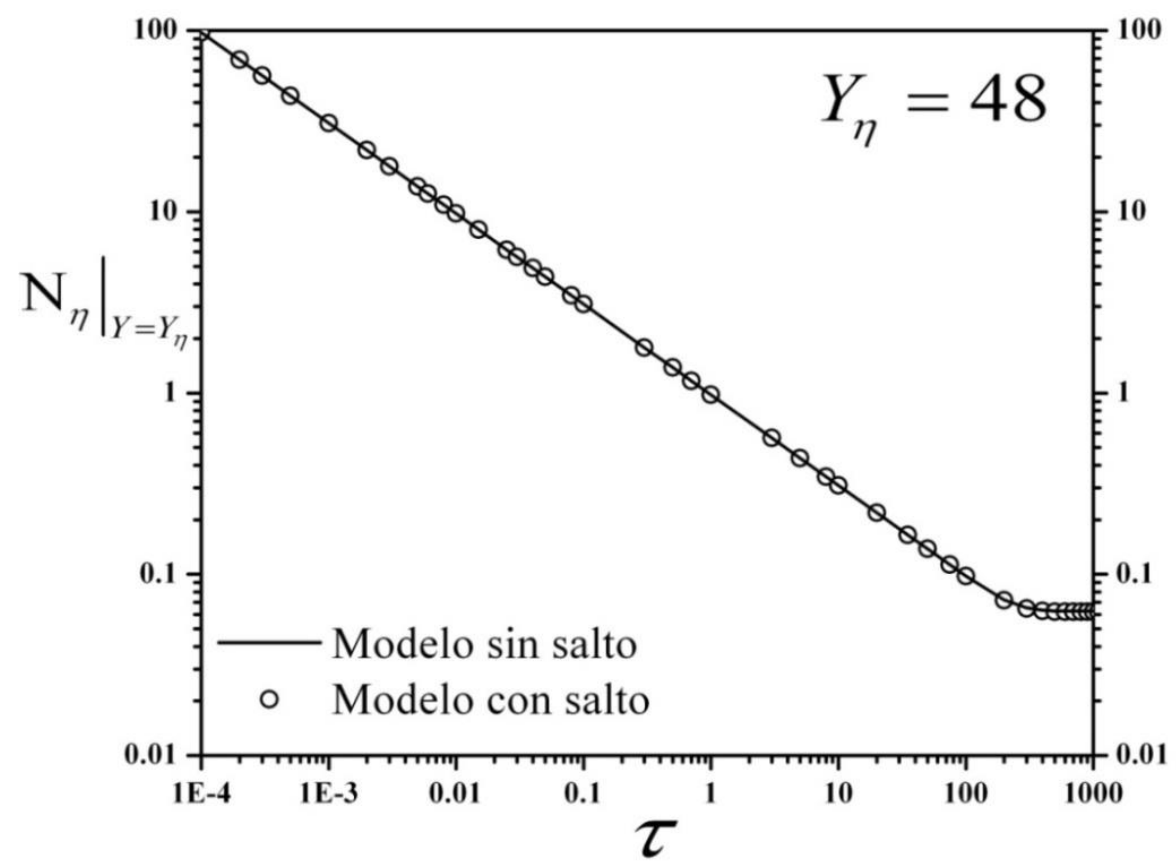

Figura 4.4 Evolución del flux de masa evaluado en $Y=Y_{\eta}$ para el Caso I-a, manteniendo constante un $\Phi_{\mathrm{I}}^{2}=5.0$ y $Y_{\eta}=48$.

\subsection{Caso I-b. La concentración en el seno del fluido externo a la fase $\eta$ depende exponencialmente del tiempo.}

Para este caso la concentración adimensional en la frontera $Y=Y_{\eta}$ se considera como una función exponencial definida anteriormente. En la Figura 4.5 se muestran los resultados de la evolución del flux de masa en $Y=Y_{\eta}$ evaluados con los dos modelos para un valor de $Y_{\eta}=5$, el \% Error del flux a un tiempo de $1 \times 10^{-5}$ es de 0.56 para este sistema, pero a medida que aumenta el tiempo y alcanzando el estado estacionario el \%Error $=8.77$. Por otro lado para un a valor de $Y_{\eta}=48$, el \% Error del flux a un tiempo de $1 \times 10^{-5}$ es de 0.49, pero a medida que aumenta el tiempo y alcanzar el estado estacionario a un $\tau>900$, el \%Error se mantiene por debajo de 0.99 como se muestra en la Figura 4.6. En ambos sistemas se mantiene constante el valor de $\Phi_{\mathrm{I}}^{2}=5$. Para obtener los resultados de la Figura 4.6 se utilizaron $4.47 \times 10^{7}$ valores propios de la serie para tiempos cortos y $4.47 \times 10^{4}$ valores propios al alcanzar el estado estacionario en el MSS. Por otro lado para el MCS se emplearon $8.81 \times 10^{6}$ valores propios para tiempos cortos y $8.81 \times 10^{3}$ valores al alcanzar el estado estacionario. 


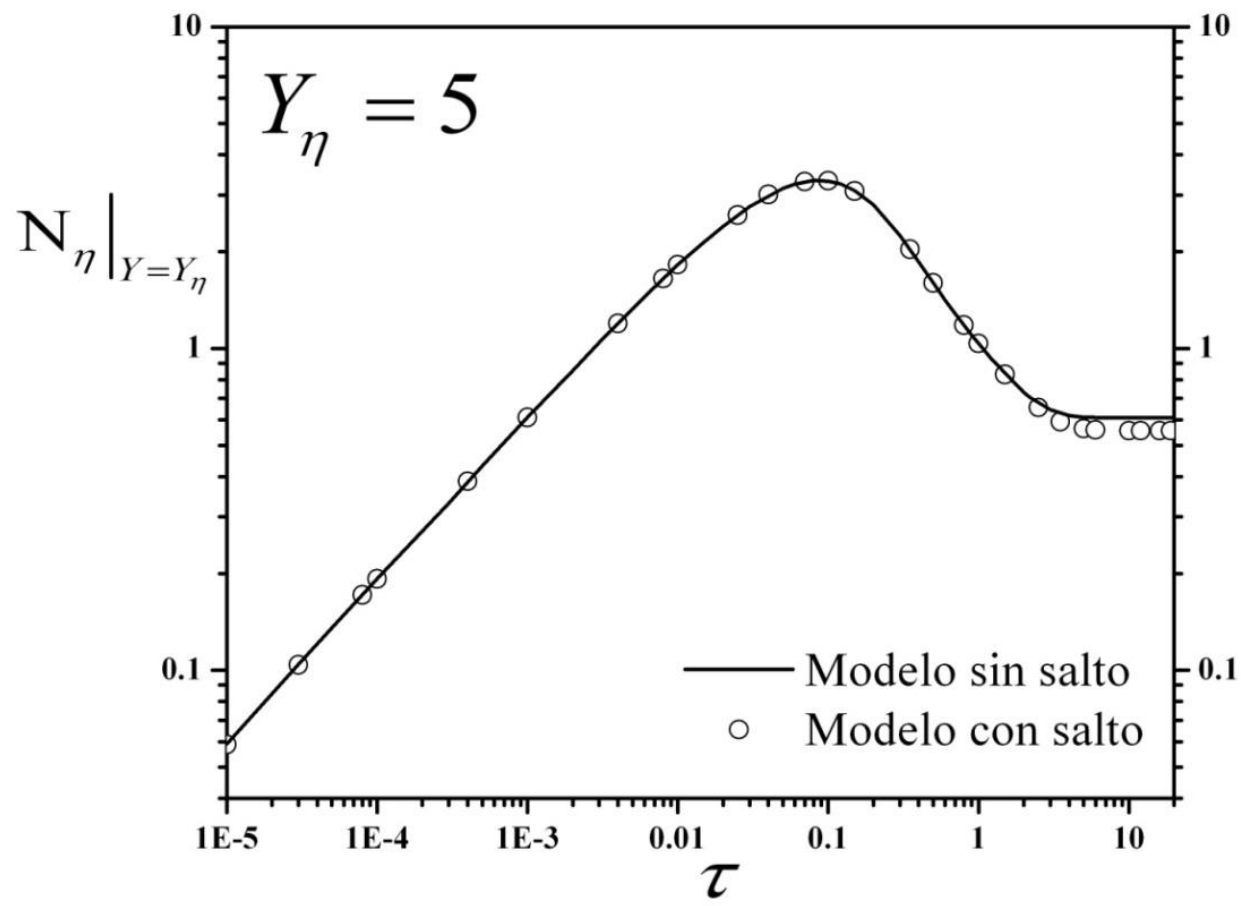

Figura 4.5 Evolución del flux de masa evaluado en $Y=Y_{\eta}$ para el Caso I-b, manteniendo constante un $\Phi_{\mathrm{I}}^{2}=5.0$ y $Y_{\eta}=5$.

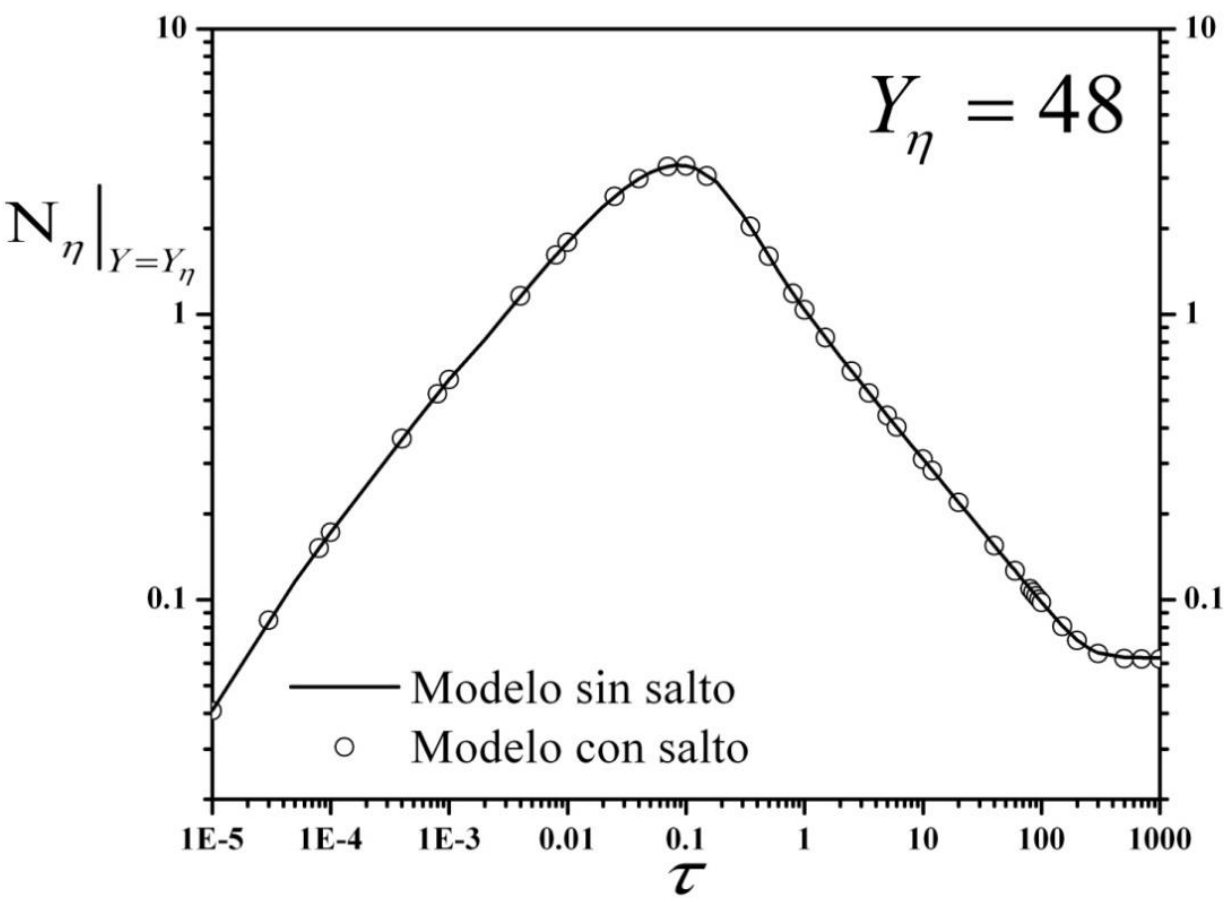

Figura 4.6 Evolución del flux de masa evaluado en $Y=Y_{\eta}$ para el Caso I-b, manteniendo constante un $\Phi_{\mathrm{I}}^{2}=5.0$ y $Y_{\eta}=48$. 
Hasta este momento se puede señalar que la condición que válida la solución aproximada que considera el salto para un sistema lineal, ya sea con fronteras fijas (Caso I-a), o con fronteras móviles (Caso I-b), es que la longitud de la fase $\eta$ sea 48 veces mayor a la longitud de la fase $I$, manteniendo los valores de los parámetros indicados en la tabla 4.1 y fijando un $\Phi_{I}^{2}=5$.

\subsection{Caso II. Reacción no lineal de segundo orden.}

En la fase $I$ se considera una reacción no lineal de segundo orden. En la Figura 4.7 se muestra las curvas del \% Error del flux de masa en $Y=Y_{\eta}$ en estado estacionario en función del $\Phi_{I}^{2}$ y $Y_{\eta}$, comparando el flux del MSS y MCS, calculados a partir de la ecuación (2.75). La expresión utilizada para evaluar el \% Error está definida por la ecuación (4.1).

Como se observa en la Figura 4.7 conforme aumenta el valor de $Y_{\eta}$, los valores del \% Error disminuyen a cualquier valor del módulo de Thiele. Sin embargo, el efecto de la reacción en la fase $I$ sobre todo el sistema es importante, ya que a valores de $0.5<\Phi_{\mathrm{I}}^{2}<5$ y $1.5<Y_{\eta}<7$ se localiza una zona donde los valores del \%Error varían en un rango de 25.3-3. Por otro lado en la sección limitada por $20<Y_{\eta}<40$ y $0.5<\Phi_{\mathrm{I}}^{2}<10$, se encuentran valores del \%Error en un rango de 2-0.7, en esta sección se observa que manteniendo un \% Error de 1.0 se requiere un valor de $Y_{\eta}=34$ a un $\Phi_{I}^{2}=5$, es decir; cuando en la fase $I$ el coeficiente de difusión es 5 veces mayor a la constante de velocidad de reacción por su longitud al cuadrado, los valores del flux de masa para el MSS y MCS serán de $\left.N_{\eta}\right|_{Y=Y_{\eta}}=0.07997$ y $\left.N_{\eta}\right|_{Y=Y_{\eta}}=0.07917$, respectivamente, solamente si la longitud de la fase $\eta$ es 34 veces mayor a la longitud de la fase $I$. De manera similar para otros valores de $\Phi_{\mathrm{I}}^{2}=0.1$ y $\Phi_{\mathrm{I}}^{2}=10$ se requieren valores de $Y_{\eta}=7$ y $Y_{\eta}=37$, respectivamente como se muestra en la Figura 4.8. Los resultados anteriores se obtuvieron con el algoritmo de Newton-Raphson multivariable, utilizando $N=10$ y $N_{I}(400-600)$ en un rango de $\Phi_{I}^{2}(10-0.1)$ para el MSS y $N=10$ a cualquier valor del módulo de Thiele para el MCS. 


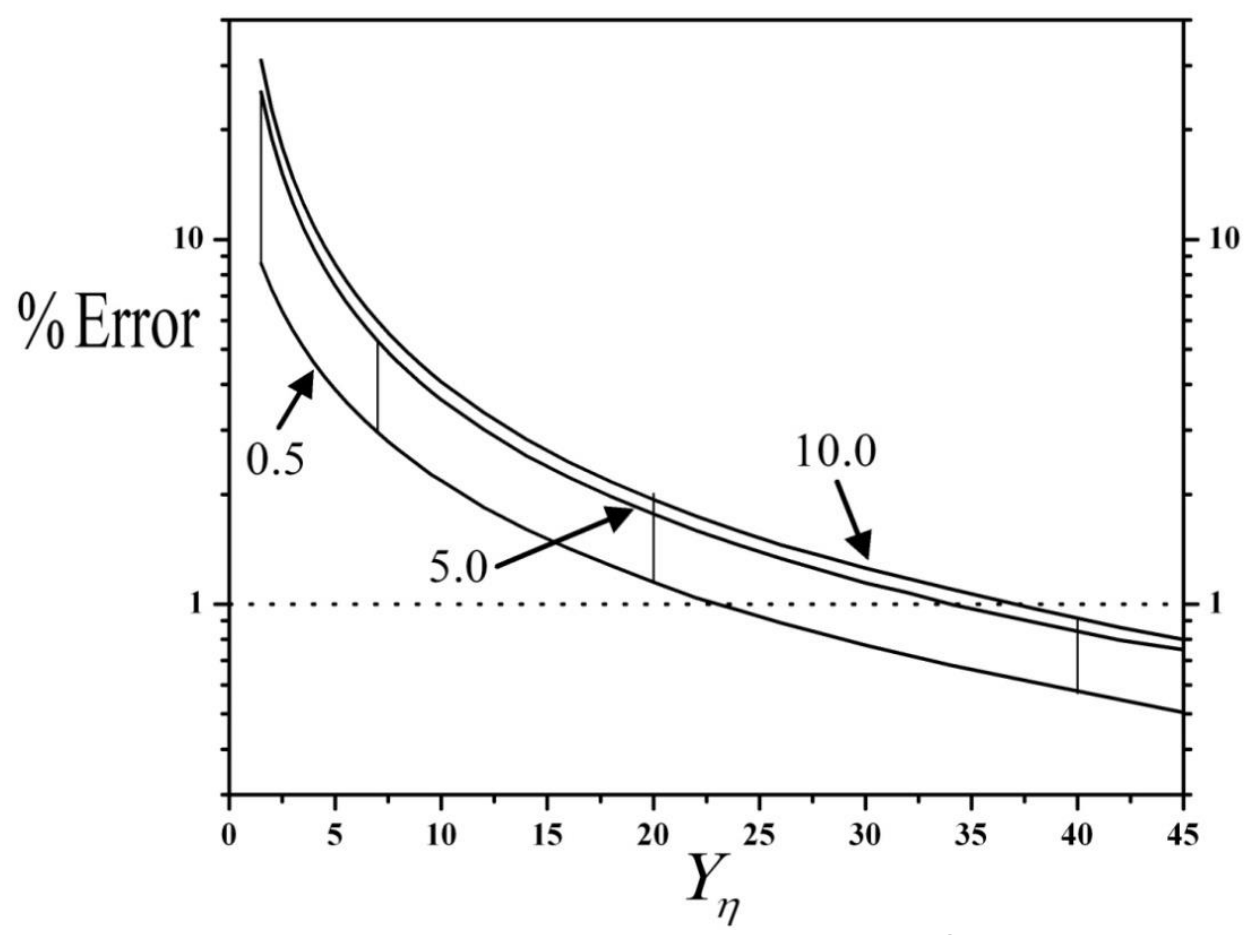

Figura 4.7 \% Error en función de $Y_{\eta}$ a diferentes valores de $\Phi_{I}^{2}$. Sistema no lineal de segundo orden.

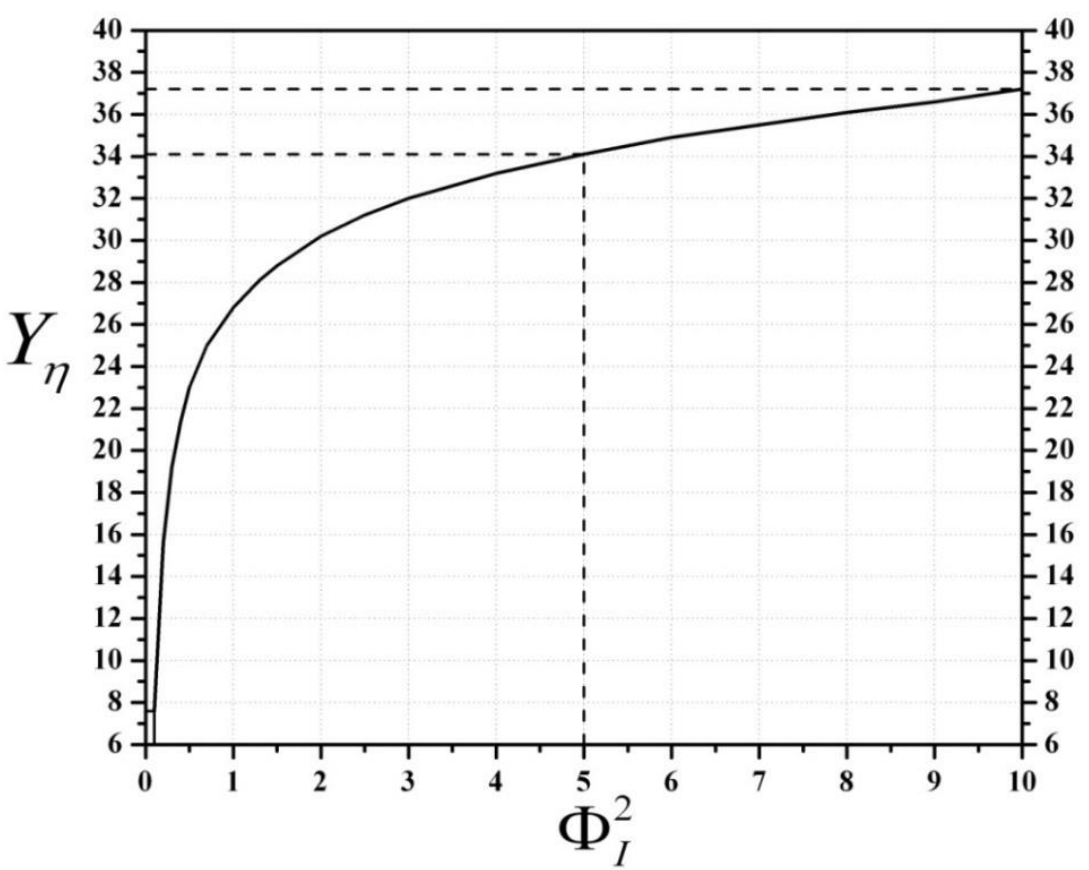

Figura 4.8 $Y_{\eta}$ requerida para mantener un \%Error de 1.0, en función de $\Phi_{I}^{2}$. Sistema no lineal de segundo orden. 


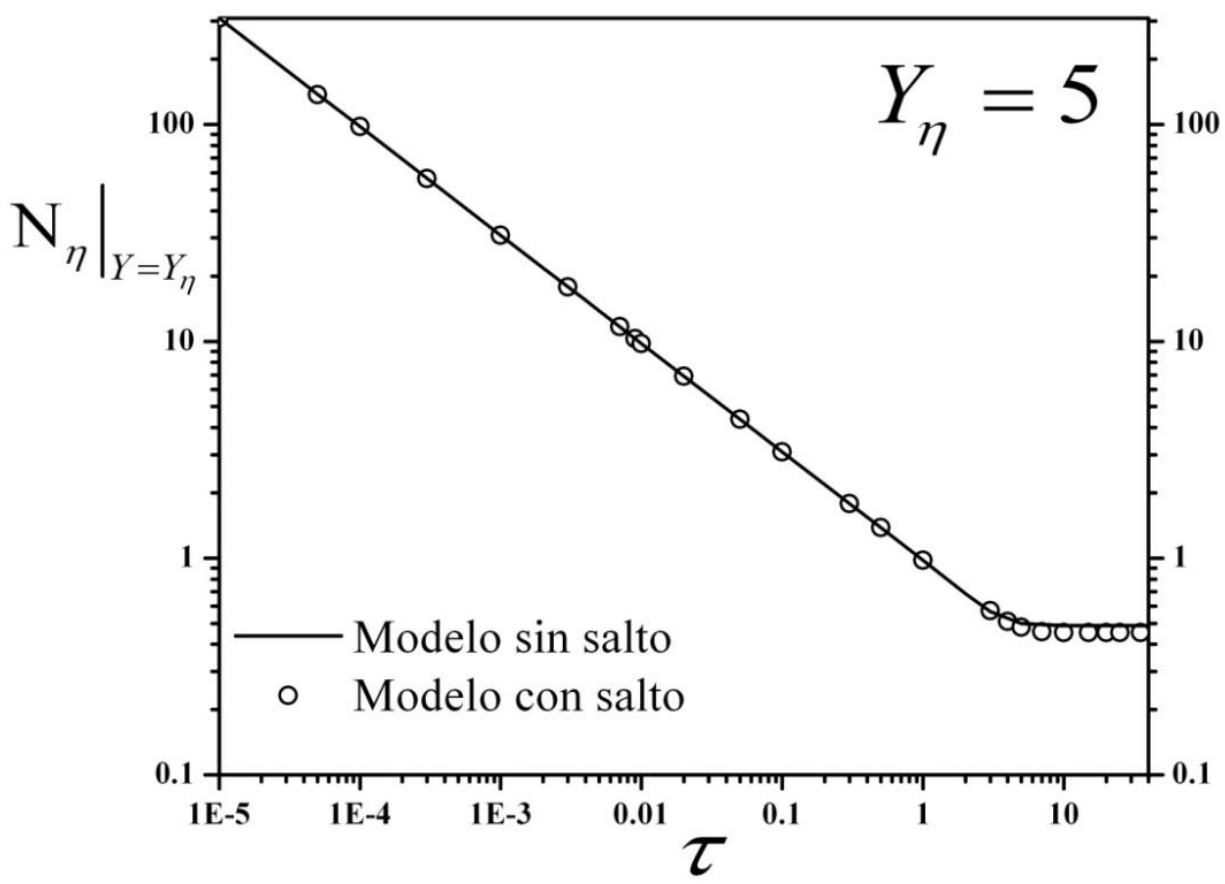

Figura 4.9 Evolución del flux de masa evaluado en $Y=Y_{\eta}$ para el sistema no lineal de segundo orden, manteniendo constante un $\Phi_{\mathrm{I}}^{2}=5.0$ y $Y_{\eta}=5$.

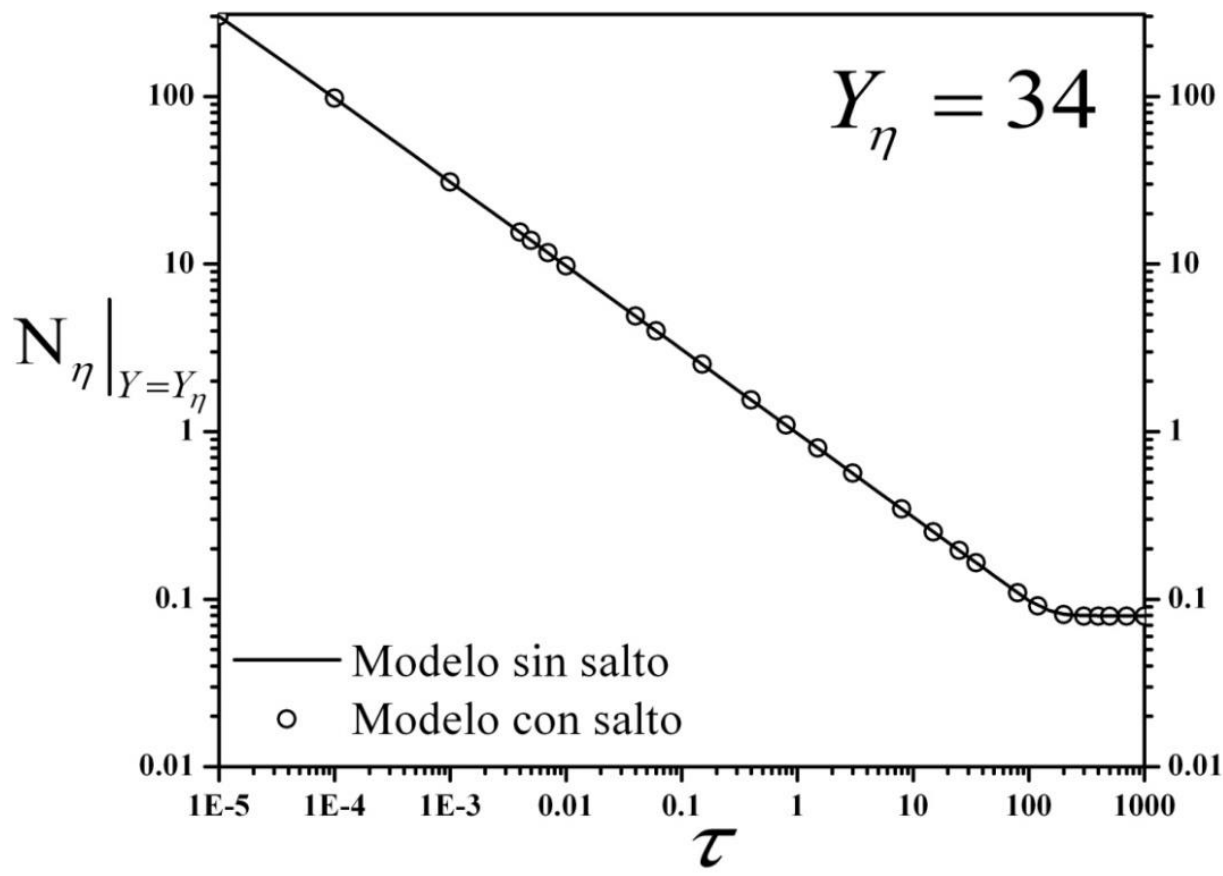

Figura 4.10 Evolución del flux de masa evaluado en $Y=Y_{\eta}$ para el sistema no lineal de segundo orden, manteniendo constante un $\Phi_{I}^{2}=5.0$ y $Y_{\eta}=34$. 
En la Figura 4.9 se muestran los resultados de la evolución del flux de masa en $Y=Y_{\eta}$ evaluados con los dos modelos para un valor de $Y_{\eta}=5$, el \% Error del flux a un tiempo de $1 \times 10^{-5}$ es de $3.5 \times 10^{-3}$ para este sistema, pero a medida que aumenta el tiempo y alcanzando el estado estacionario el \%Error $=7.47$. Por otro lado para un a valor de $Y_{\eta}=$ 34 , el \% Error del flux a un tiempo de $1 \times 10^{-5}$ es de 0.11 , pero al alcanzar el estado estacionario a un $\tau>500$, el \% Error se mantiene por debajo de 1.0 como se muestra en la Figura 4.10. En ambos sistemas se mantiene constante el valor de $\Phi_{I}^{2}=5.0$.

En la Tabla 4.2 se muestran las condiciones de la evaluación numérica en estado transitorio de los dos modelos, para el MSS se mantuvo constante $N_{I}=20$ y $h$ es el tamaño de paso del tiempo. Además en la última columna de la tabla, se observan en términos de $\%$, el tiempo de computo que solamente es utilizado para resolver el MCS, con respecto al tiempo de computo total que es requerido para evaluar el MSS, manteniendo constante los parámetros de la Tabla 4.1, considerando un $\Phi_{\mathrm{I}}^{2}=5.0$ y $Y_{\eta}=48$.

Tabla 4.2 Tiempos de cómputo utilizados para la evaluación en estado transitorio de los dos modelos. Caso II.

\begin{tabular}{|c|c|c|c|}
\hline$\tau$ & $N$ & $h$ & $\%$ \\
\hline $1 \times 10^{-5}$ & $2 \times 10^{4}$ & $1 \times 10^{-7}$ & 54.7 \\
\hline 0.01 & 500 & $1 \times 10^{-4}$ & 55 \\
\hline 1 & 100 & $1 \times 10^{-3}$ & 50 \\
\hline 500 & $30^{*} / 10^{* *}$ & $\left(1 \times 10^{-3}\right)^{*} / 1^{* *}$ & 0.2 \\
\hline \multicolumn{4}{|c}{${ }^{*} \mathrm{MSS} / * * \mathrm{MCS}$} \\
\end{tabular}

\subsection{Caso III. Cinética de Michaelis Menten.}

En la fase $I$ se considera una reacción enzimática de Michaelis-Menten. El termino de reacción en la ecuación (3.31) para la fase $I$, depende de dos parámetros adimensionales $\left(\kappa_{M}, \Phi_{\mathrm{M}}^{2}\right)$ que reflejan la acción de una enzima. La constante $V_{\max }$ es una medida de la eficiencia de operación, es el límite superior de rendimiento de cierta cantidad de enzima, por lo que suele usarse para comparar enzimas diferentes. Mientras que la constante de Michaelis-Menten representa la cantidad de sustrato necesaria para fijarse a la mitad de la enzima disponible y producir la mitad de la velocidad máxima. Por otro lado, los valores de $K_{m}$ pueden usarse para evaluar la especificidad de acción de una determinada enzima hacia 
los sustratos similares. La regla general que se sigue es: en cuanto más bajo sea el valor de $K_{m}$ mejor será el sustrato. En la Figura 4.11 se muestran las curvas de velocidad inicial adimensional a diferentes valores de $\kappa_{M}$ donde se observa que a medida que disminuyen los valores de $K_{m}$, se alcanza la $V_{\max }$ fijada por un $\Phi_{\mathrm{M}}^{2}=5.0$ a concentraciones bajas.

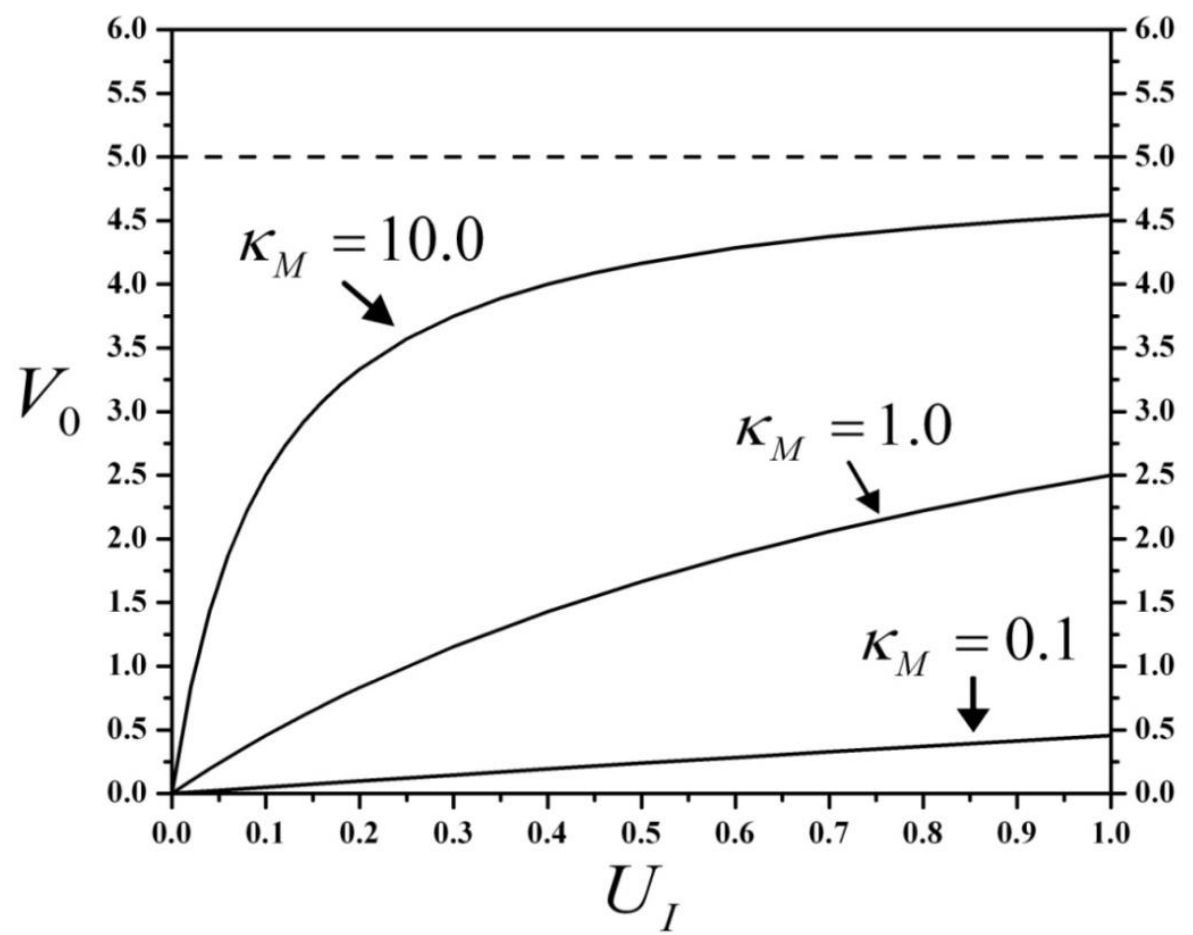

Figura 4.11 Grafica de $V_{0}$ en función de $U_{I}$ para una reacción catalizada por una enzima diferentes valores de $\kappa_{M}$.

Con base en lo anterior, en la Figura 4.12 se muestran las curvas del \% Error en función de $Y_{\eta}$, a diferentes valores de $\Phi_{\mathrm{M}}^{2}$, fijando una $\kappa_{M}=0.1$, donde se aprecia que a valores de $\Phi_{\mathrm{M}}^{2}<5.0$ existe un máximo del \% Error y conforme los valores de $Y_{\eta}$ aumentan la curva se normaliza. Por otro lado en la sección limitada por $40<Y_{\eta}<80$ y a un $0.8<\Phi_{\mathrm{M}}^{2}<$ 10, se encuentran valores de \%Error en un rango de 2.01-0.64, en esta sección se observa que manteniendo un \% Error de 1.0 se requiere un valor de $Y_{\eta}=54$ a un $\Phi_{\mathrm{M}}^{2}=1.0$, es decir; cuando en la fase $I$ el efecto difusivo es igual al termino reactivo, los valores del flux de masa para el MSS y MCS serán de $\left.N_{\eta}\right|_{Y=Y_{\eta}}=0.0559$ y $\left.N_{\eta}\right|_{Y=Y_{\eta}}=0.0553$, respectivamente, solamente si la longitud de la fase $\eta$ es 54 veces mayor a la longitud de la fase $I$. De manera similar para otros valores de $\Phi_{\mathrm{M}}^{2}=0.5$ y $\Phi_{\mathrm{M}}^{2}=10.0$ se requieren 
valores de $Y_{\eta}=44$ y $Y_{\eta}=80$, respectivamente como se muestra en la Figura 4.13. Los resultados anteriores se obtuvieron con el algoritmo de Newton-Raphson multivariable, utilizando $N=10$ y $N_{I}(100-200)$ en un rango de $\Phi_{\mathrm{M}}^{2}(10-0.5)$ para el MSS y $N=5$ a cualquier valor del módulo de Thiele para el MCS.

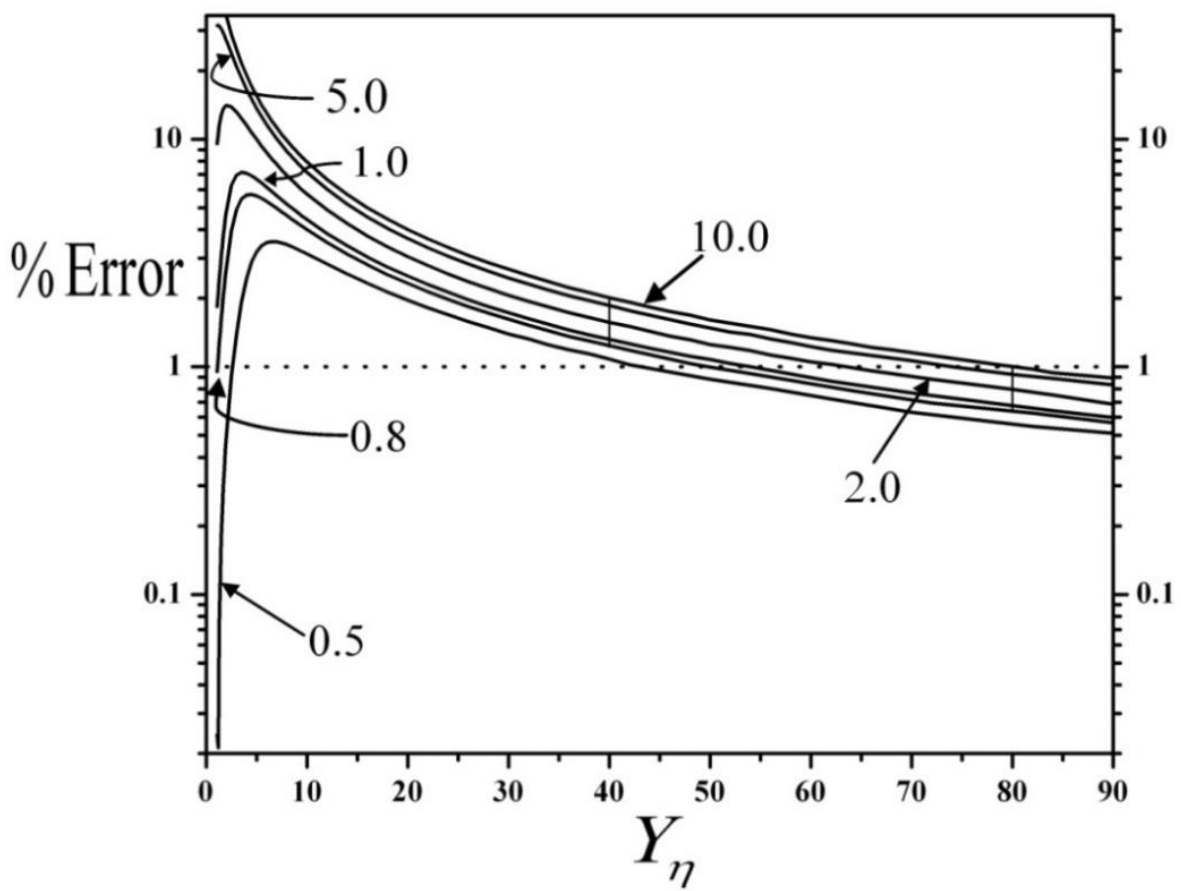

Figura 4.12 \% Error en función de $Y_{\eta}$ a diferentes valores de $\Phi_{M}^{2}$. Sistema que considera una cinética de Michaelis Menten.

En la Figura 4.14 se muestran los resultados de la evolución del flux de masa en $Y=Y_{\eta}$ evaluados con los dos modelos para un valor de $Y_{\eta}=5$, el $\%$ Error del flux a un tiempo de $1 \times 10^{-5}$ es de 0.02 para este sistema, pero a medida que aumenta el tiempo y alcanzando el estado estacionario el \%Error $=6.68$. Por otro lado para un a valor de $Y_{\eta}=54$, el $\%$ Error del flux a un tiempo de $1 \times 10^{-5}$ es de 0.15 , pero al alcanzar el estado estacionario a un $\tau>500$ el valor del \%Error es 1.0 como se muestra en la Figura 4.15. En ambos sistemas se mantiene constante el valor de $\Phi_{\mathrm{M}}^{2}=1.0$. 


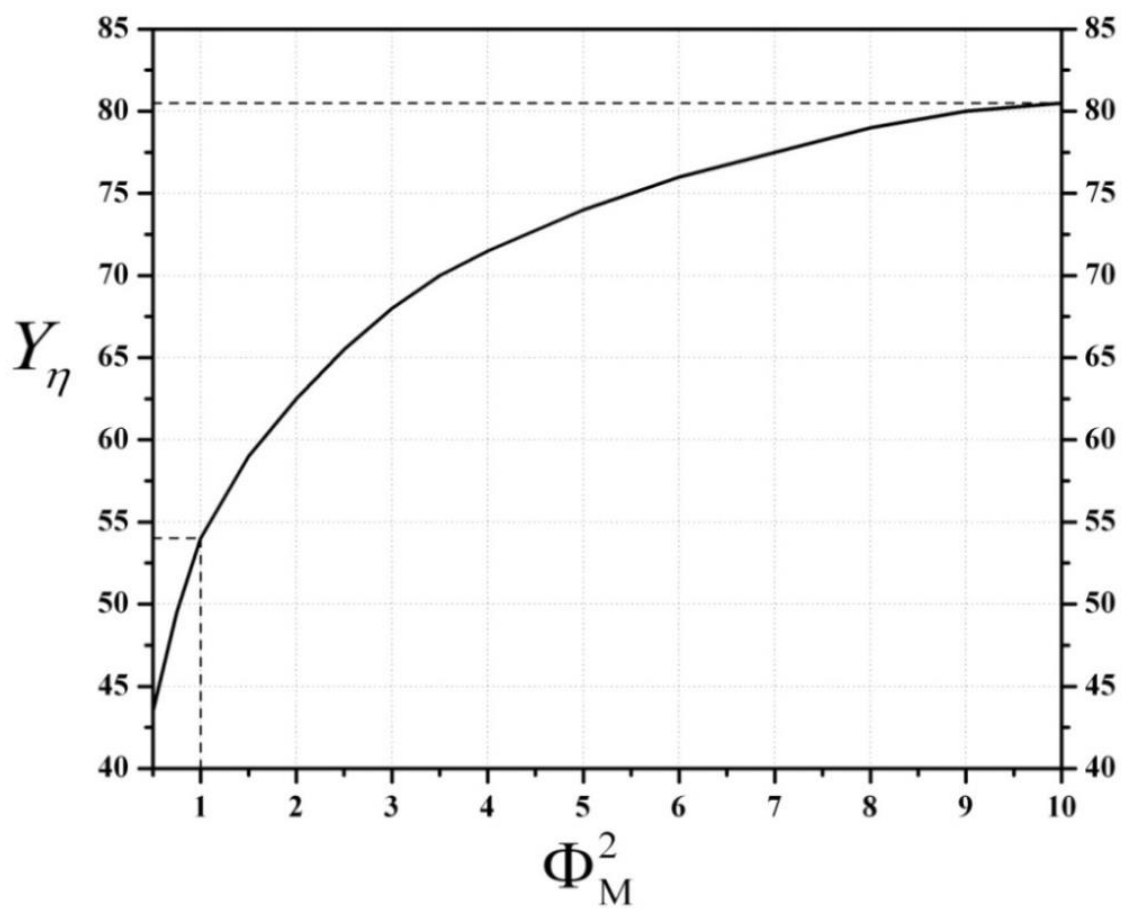

Figura 4.13 $Y_{\eta}$ requerida para mantener un \%Error de 1.0, en función de $\Phi_{M}^{2}$. Sistema que considera una cinética de Michaelis Menten.

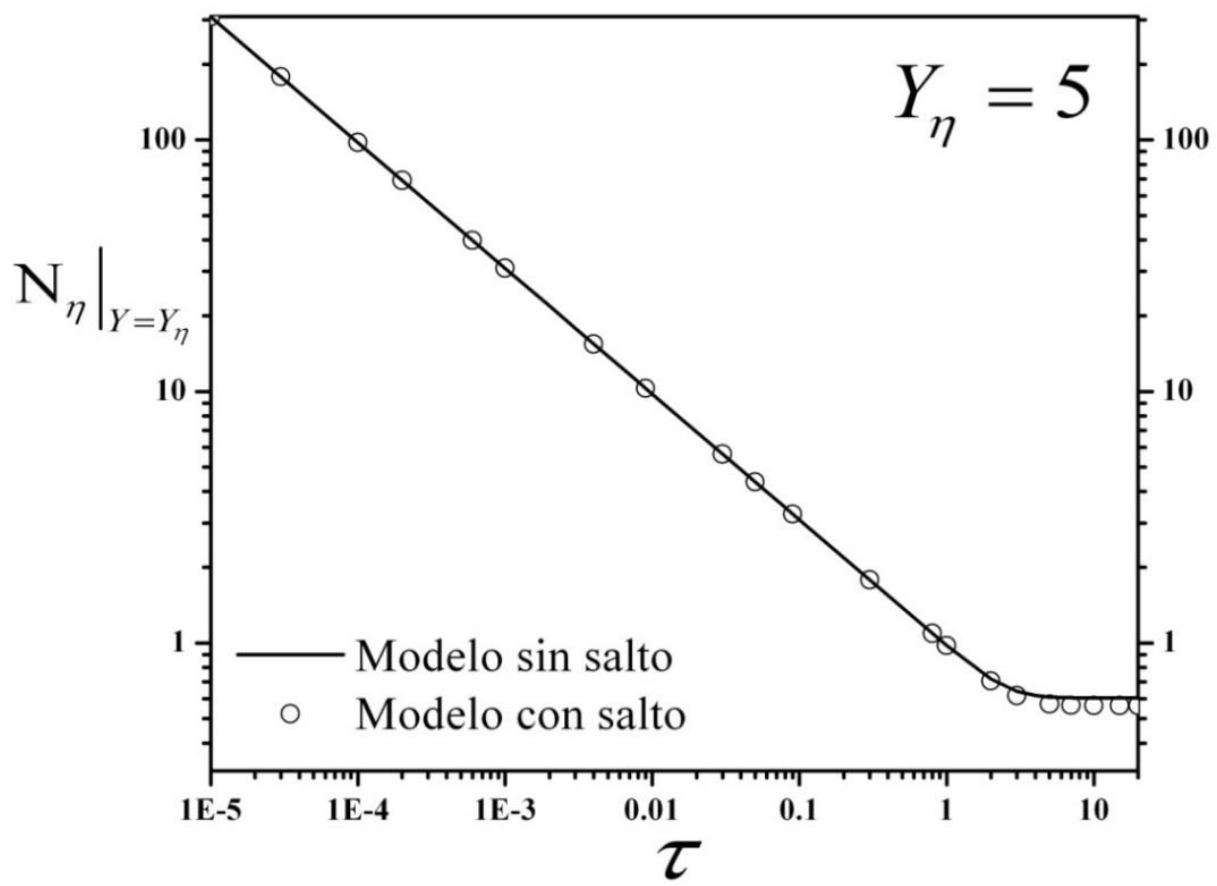

Figura 4.14 Evolución del flux de masa en $Y=Y_{\eta}$ para el sistema que considera una cinética de Michaelis Menten, manteniendo constante un $\Phi_{M}^{2}=1.0$ y $Y_{\eta}=5$. 


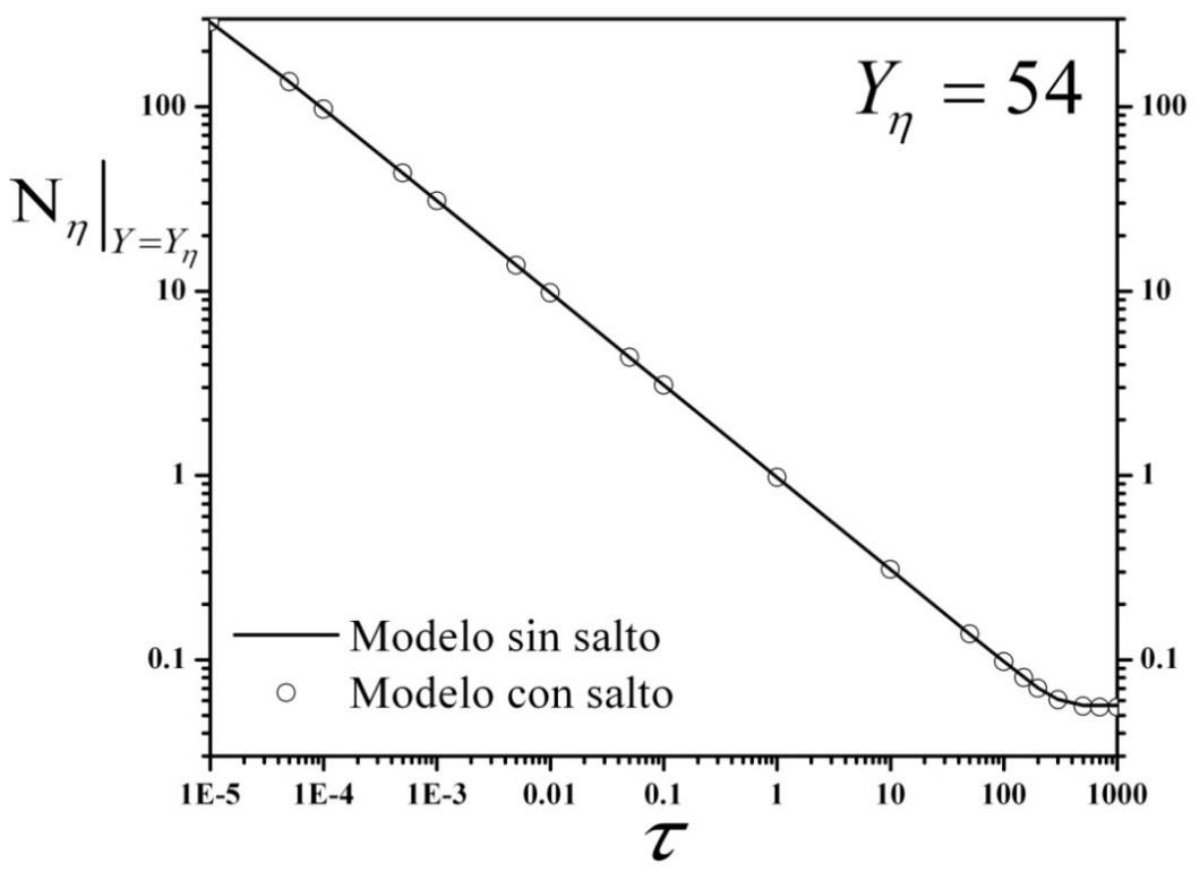

Figura 4.15 Evolución del flux de masa en $Y=Y_{\eta}$ para el sistema que considera una cinética de Michaelis Menten, manteniendo constante un $\Phi_{M}^{2}=1.0$ y $Y_{\eta}=54$.

En la Tabla 4.3 se muestran las condiciones de la evaluación numérica en estado transitorio de los dos modelos, para el MSS se mantuvo constante $N_{I}=20$. Además en la última columna de la tabla, se observan en términos de \%, el tiempo de computo que solamente es utilizado para resolver el MCS, con respecto al tiempo de computo total que es requerido para evaluar el MSS, manteniendo constante los parámetros de la Tabla 4.1, considerando un $\Phi_{M}^{2}=1.0, \kappa_{M}=1.0$ y $Y_{\eta}=54$.

Tabla 4.3 Tiempos de cómputo utilizados para la evaluación en estado transitorio de los dos modelos. Caso III.

\begin{tabular}{|c|c|c|c|}
\hline$\tau$ & $N$ & $h$ & $\%$ \\
\hline $1 \times 10^{-5}$ & $2.5 \times 10^{4}$ & $1 \times 10^{-8}$ & 51.7 \\
\hline 0.01 & 5000 & $1 \times 10^{-5}$ & 51.84 \\
\hline 1 & 200 & 0.01 & 36.8 \\
\hline 500 & $50^{*} / 30^{* *}$ & $\left(1 \times 10^{-3}\right)^{*} / 0.1^{* *}$ & 0.53 \\
\hline \multicolumn{4}{|c}{$* \mathrm{MSS} / * * \mathrm{MCS}$} \\
\hline
\end{tabular}


Para finalizar este capítulo se puede señalar que la condición que válida la solución aproximada que considera el salto para todos los casos es la longitud característica de la fase $\eta$, y como se puede observar, en sistemas que tengan una $Y_{\eta}$ mayor a 80 , en un rango de $0.1<\Phi_{\mathrm{I}}^{2}<10$, se puede asegurar que el \% Error del flux de masa evaluado con el MSS y el MCS, tendrá un máximo de uno, para todos los casos de estudio, manteniendo los valores de los parámetros indicados en la tabla 4.1. Además en la tabla 4.4, a manera de resumen se muestran en términos de $\%$, el tiempo de computo que solamente es utilizado para resolver el MCS, con respecto al tiempo de computo total que es requerido para evaluar el MSS en estado estacionario para todos los casos, considerando un $\Phi_{\mathrm{I}}^{2}=5.0$ para los casos I-a, I-b, II y un $\Phi_{\mathrm{M}}^{2}=1.0$ para el caso III.

Tabla 4.4 Tiempos de cómputo utilizados para la evaluación en estado estacionario de los dos modelos.

\begin{tabular}{|c|c|c|c|c|}
\hline Casos & $\mathrm{I}-\mathrm{a}\left(Y_{\eta}=48\right)$ & $\mathrm{I}-\mathrm{b}\left(Y_{\eta}=48\right)$ & $\mathrm{II}\left(Y_{\eta}=34\right)$ & $\mathrm{III}\left(Y_{\eta}=54\right)$ \\
\hline$\%$ & 24.9 & 8.3 & 5.6 & 22.1 \\
\hline
\end{tabular}




\section{CONCLUSIONES}

En este trabajo se desarrolló una solución analítica para una región compuesta por dos fases, considerando una reacción lineal de primer orden en una de ellas, utilizando conceptos matemáticos sencillos como la Identidad de Green y el método de superposición, guardando la generalidad de la solución para diferentes condiciones. Asimismo se analizó y comparó con la solución aproximada del sistema, bajo la cual se obtiene con el reemplazo de una condición de frontera apropiada por la ecuación gobernante de la fase $I$. De manera similar al caso lineal, se estudiaron las condiciones para dos sistemas no lineales.

La propuesta de reemplazo de la ecuación de transporte de masa de la fase I por una condición de frontera apropiada, es válida si la longitud característica de esta fase tiene un valor con dos órdenes de magnitud como mínimo para todos los casos estudiados en este trabajo, a diferentes valores del módulo de Thiele.

Para el caso lineal, se demostró que la solución aproximada es válida para sistemas con o sin fronteras móviles, mediante el error del flux de masa entre los dos modelos. Por otro lado para los problemas no lineales, la solución aproximada resultó ser de gran utilidad ya que el tiempo de cómputo utilizado se redujo, obteniendo longitudes características de la fase $\eta$, que se encuentran dentro del rango reportado en la literatura, como en los casos estudiados por Asprion y Torres.

Por último también se demostró que para el conjunto de valores de los parámetros y del módulo de Thiele utilizados en la solución dinámica del sistema, solo es necesario resolver los dos modelos en estado estacionario para encontrar la longitud que es indispensable para que los fluxes de masa sean similares y con esta condición resolver únicamente el modelo con salto en estado transitorio para poder evaluar el flux de masa a cualquier tiempo y así evitar obtener la solución en estado transitorio del modelo original. 


\section{TRABAJO FUTURO}

Una posible perspectiva de este trabajo es que el método aproximado basado en el uso de la condición de salto como una de sus fronteras, se puede aplicar a sistemas con mayor complejidad, por ejemplo en reactores con membrana usualmente existen tres fases donde en una de ellas ocurre una reacción química que favorece el transporte de algún soluto.

El modelo unidimensional en estado transitorio para un sistema de tres fases con sus respectivas condiciones iníciales y de frontera, considerando transporte de masa por difusión y el aumentado por una reacción lineal en la fase membrana $(I)$ y además el transporte de masa por difusión en las fases $(\eta)$ y $(\omega)$ localizadas alrededor de la fase membrana, puede ser de la siguiente forma:

$$
\text { En } \quad y=L_{\eta} \quad C_{\eta}^{F}=f_{\eta}(t) \text { para } t>0
$$

Fase $\eta$

$$
\begin{gathered}
\frac{\partial C_{\eta}}{\partial t}=D_{\eta} \frac{\partial^{2} C_{\eta}}{\partial y^{2}} \quad \text { para } \quad L_{I}<y<L_{\eta} \\
\text { En } y=L_{I} \quad C_{I}^{e}=C_{\eta}^{e} K_{e q}^{\eta I} \quad \text { para } t>0 \\
\text { En } y=L_{I} \quad D_{\eta} \frac{\partial C_{\eta}^{e}}{\partial y}=D_{I} \frac{\partial C_{I}^{e}}{\partial y} \text { para } t>0
\end{gathered}
$$

Fase $I$

$$
\begin{gathered}
\frac{\partial C_{I}}{\partial t}=D_{I} \frac{\partial^{2} C_{I}}{\partial y^{2}}-k_{I} C_{I} \quad \text { para }-L_{I}<y<L_{I} \\
\text { En } \quad y=-L_{I} \quad C_{I}^{e}=C_{\omega}^{e} K_{e q}^{\omega I} \text { para } t>0 \\
\text { En } \quad y=-L_{I} \quad D_{I} \frac{\partial C_{I}^{e}}{\partial y}=D_{\omega} \frac{\partial C_{\omega}^{e}}{\partial y} \text { para } t>0
\end{gathered}
$$

Fase $\omega$

$$
\begin{gathered}
\frac{\partial C_{\omega}}{\partial t}=D_{\omega} \frac{\partial^{2} C_{\omega}}{\partial y^{2}} \quad \text { para } \quad L_{\omega}<y<-L_{I} \\
\text { En } y=L_{\omega} \quad C_{\omega}^{F}=f_{\omega}(t) \text { para } t>0
\end{gathered}
$$


En este caso se buscaría reemplazar la ecuación gobernante de la fase $I$ de espesor pequeño, por una condición de salto, que acople las dos fases $\eta$ у $\omega$. Para otros problemas, las condiciones de frontera en los extremos de las fases $\eta$ y $\omega$ pueden considerar la difusión de masa en la superficie a través de un coeficiente de transferencia de masa.

Finalmente es importante señalar que los resultados obtenidos en este trabajo deberán esperar una verificación con una simulación que incluya datos experimentales; es decir, esta tesis, solo es el primer paso en la búsqueda de soluciones aproximadas, que permitan, en alguna medida, el cálculo del flux de masa de sistemas dinámicos de varias fases. 


\section{BIBLIOGRAFÍA}

[1] Agar, D., 1999, "Multifunctional reactors: Old preconceptions an new dimensions", Chemical Engineering Science 54 , 1299-1305.

[2] Aguilar Madera, C., Valdés Parada, F., Goyeau, B., Ochoa Tapia, J., 2011, "Onedomain approch for heat transfer between a porous medium and a fluid", International Journal of Heat and Mass Transfer 54 , 2089-2099.

[3] Asprion, N., 2006, "Nonequilibrium Rate Based Simulation of Reactive Systems: Simulation Model, Heat Transfer and Influence of Film Discretization", Industrial and Engineering Chemistry Research 45 , 2054-2069.

[4] Beavers, G., Joseph, D., 1967, "Boundary conditions at a naturally permeable wall", Journal of Fluids Mechanics 30 , Part. I , 197-207.

[5] Dautzenberg, F., Mukherjee, M., 2001, "Process intensification using multifunctional reactors", Chemical Engineering Science 56 , 251-267.

[6] De Falco, M., Marrelli, L., Iaquaniello, G., 2011, Membrane Reactors for Hydrogen Production Processes, New York: Springer.

[7] Elsner, M., Dittrich, C., Agar, D., 2002, "Adsorptive reactors for enhancing equilibrium gas-phase reactions", Chemical Engineering Science 57, 1607-1619.

[8] Fay, T., Kloppers, P., 2001, "The Gibbs' phenomenon", International Journal of Mathematical Education in Science and Technology 32 , No. 1 , 73-89.

[9] Gaidhani, H., Tolani, V., Pangarkar, K., Pangarkar, V., 2002, "Intensification of enzymatic hydrolysis of penicillin G.", Chemical Engineering Science 57 , 1985-1992. 
[10] Gray, W. G., 1975, "A derivation of the equations for multi-phase transport", Chemical Engineering Science 30 , 229-233.

[11] Haber, S., Mauri, R., 1983, "Boundary conditions for Darcy's flow through porous media", International Journal of Multiphase flow 9 , No. 5 , 561-574.

[12] Kening, E., Górak, A., 1995, "A film model based approach for simulation of multicomponent reactive separation", Chemical Engineering Processing 34, 97-103.

[13] Kreul, L., Górak, A., Barton, P., 1999, "Modeling of homogeneous reactive separation processes in packed columns", Chemical Engineering Science 54, 19-34.

[14] Lewis, W., Whitman, W., 1924, "Principles of gas absorption", Industrial Engineering Chemistry 16, 1215-1220.

[15] Lin, Y.-M., Liu, S.-L., Chuang, C.-H., Chu, Y.-T., 2003, "Effect of incipient removal of hydrogen through palladium membrane on the conversion of methane steam reforming experimental and modeling", Catalysis Today 82, 127-139.

[16] Mazzotti, M., Kruglov, A., Neri, B., Gelosa, D., Massimo, M., 1996, "A continuous chromatographic reactor: SMBR", Chemical Engineering Science 51, No. 10, 1827-1836.

[17] Noeres, C., Kenig, E., Górak, A., 2003, "Modelling of reactive separation processes reactive absorption and reactive destillation", Chemical Engineering and Processing $\mathbf{4 2}$, $157-178$.

[18] Nowak, U., Frauhammer, J., Nieken, U., 1996, "A fully adaptive algorithm for parabolic partial differential equations in one space dimension", Computers and Chemical Engineering 20 , No. 5 , 547-561.

[19] Ochoa Tapia, J., Whitaker, S., 1995, "Momentum transfer at the boundary between a porous medium and a homogeneous fluid- I. Theorical development", International Journal of Heat and Mass Transfer 38 , No. 14 , 2635-2646. 
[20] Sanchez Marcano, J., Tsotsis, T., 2002, Catalytic Membranes and Membrane Reactors, Weinheim: Wiley-VCH.

[21] Schmidt-Traub, H., Górak, A., 2006, Integrated Reaction and Separation Operations, New York: Springer.

[22] Seader, J., 1989, "The rate-based approach for modelling staged separations", Chemical Engineering Progress 85 , No. 10, 41-49.

[23] Torres, M., Sanchez, J., Dalmon, J., Bernauer, B., Lieto, J., 1994, "Modeling and Simulation of a Three-Phase Catalytic Membrane Reactor for Nitrobenzene Hydrogenation", Industrial and Engineering Chemistry Research 33 , 2421-2425.

[24] Valdés Parada, F., Benoit, G., Ochoa Tapia, J., 2006, "Diffusive mass transfer between a microporous medium and a homogeneous fluid: Jump Boundary Conditions", Chemical Engineering Science 61, 1692-1704.

[25] Valdés Parada, F., Ochoa Tapia, J., Alvarez Ramirez, J., 2007, "Diffusive mass transport in the fluid porous medium inter-region: Closure problem solution for the one domain approach", Chemical Engineering Science 62 , 6054-6068.

[26] Westerterp, K., 1992, "Multifunctional reactors", Chemical Engineering Science 47 , No. 9-11, 2195-2206.

[27] Whitaker, S., 1999, The method of volume averaging, Dordrecht: Kluwer Academic Publishers.

[28] Wood, B., Quintard, M., Whitaker, S., 2000, "Jump condition at non uniform boundaries: the catalytic surface", Chemical Engineering Science 55 , 5231-5245. 


\section{APÉNDICE A}

\section{Detalles de la adimensionalizacion del sistema de dos fases}

Utilizando las definiciones de las variables adimensionales (Tabla 2.1) se lleva a cabo la adimensionalizacion de las ecuaciones diferenciales (2.1) y (2.2) para obtener las ecuaciones (2.8) y (2.9) como sigue:

$$
\begin{gathered}
\frac{\partial C_{\eta}}{\partial t}=C_{\eta}^{*} \frac{\partial U_{\eta}}{\partial \tau} \frac{\partial \tau}{\partial t}=\frac{C_{\eta}^{*} D^{*}}{L^{2}} \frac{\partial U_{\eta}}{\partial \tau} \\
D_{\eta} \frac{\partial}{\partial y}\left(\frac{\partial C_{\eta}}{\partial y}\right)=D_{\eta} C_{\eta}^{*} \frac{\partial}{\partial y}\left(\frac{\partial U_{\eta}}{\partial Y}\right) \frac{1}{L}=D_{\eta} C_{\eta}^{*} \frac{\partial}{\partial y}\left(\frac{\partial U_{\eta}}{\partial Y}\right) \frac{\partial Y}{\partial Y} \frac{1}{L}=\frac{D_{\eta} C_{\eta}^{*}}{L^{2}} \frac{\partial^{2} U_{\eta}}{\partial Y^{2}} \\
\frac{C_{\eta}^{*} D^{*}}{L^{2}} \frac{\partial U_{\eta}}{\partial \tau}=\frac{D_{\eta} C_{\eta}^{*}}{L^{2}} \frac{\partial^{2} U_{\eta}}{\partial Y^{2}} \\
\frac{\partial C_{I}}{\partial t}=C_{I}^{*} \frac{\partial U_{I}}{\partial \tau} \frac{\partial \tau}{\partial t}=\frac{C_{I}^{*} D^{*}}{L^{2}} \frac{\partial U_{I}}{\partial \tau} \\
D_{I} \frac{\partial U_{\eta}}{\partial y}\left(\frac{\partial C_{I}}{\partial y}\right)=D_{I} C_{I}^{*} \frac{\partial}{\partial y}\left(\frac{\partial U_{I}}{\partial Y}\right) \frac{1}{L}=D_{I} C_{I}^{*} \frac{\partial}{\partial y}\left(\frac{\partial U_{I}}{\partial Y}\right) \frac{\partial Y}{\partial Y} \frac{1}{L}=\frac{D_{I} C_{I}^{*}}{L^{2}} \frac{\partial^{2} U_{I}}{\partial Y^{2}} \\
\frac{C_{I}^{*} D^{*}}{L^{2}} \frac{\partial U_{I}}{\partial \tau}=\frac{D_{I} C_{I}^{*}}{L^{2}} \frac{\partial^{2} U_{I}}{\partial Y^{2}}-k_{I} C_{I}^{*} U_{I} \\
\frac{\partial U_{I}}{\partial \tau}=\alpha_{I} \frac{\partial^{2} U_{I}}{\partial Y^{2}}-\Phi_{I}^{2} U_{I}
\end{gathered}
$$


Procediendo de la misma forma, adimensionalizamos las condiciones de frontera, ecuaciones, (2.3)-(2.6), y la condición inicial, ecuación (2.7), para obtener las ecuaciones (2.10)-(2.14) como sigue:

En $Y=Y_{\eta}$

$$
\begin{gathered}
C_{\eta}^{*} U_{\eta}=C_{\eta}^{B}(t) \\
U_{\eta}=F_{\eta}(\tau)
\end{gathered}
$$

En $Y=1$

$$
\begin{gathered}
C_{I}^{e^{*}} U_{I}=C_{\eta}^{e^{*}} U_{\eta} K_{e q}^{\eta I} \\
C_{I}^{e^{*}}=C_{\eta}^{e^{*}} K_{e q}^{\eta I} \\
U_{I}=U_{\eta}
\end{gathered}
$$

En $Y=1$

$$
\begin{gathered}
D_{\eta} \frac{\partial C_{\eta}^{e}}{\partial y}=D_{\eta} C_{\eta}^{e^{*}} \frac{\partial U_{\eta}}{\partial Y} \frac{\partial Y}{\partial y}=\frac{D_{\eta} C_{\eta}^{e^{*}}}{L} \frac{\partial U_{\eta}}{\partial Y} \\
\frac{D_{\eta} C_{\eta}^{e^{*}}}{L} \frac{\partial U_{\eta}}{\partial Y}=\frac{D_{I} C_{I}^{e^{*}}}{L} \frac{\partial U_{I}}{\partial Y} \\
\kappa_{\eta} \frac{\partial U_{\eta}}{\partial Y}=\frac{\partial U_{I}}{\partial Y}
\end{gathered}
$$

En $Y=0$

$$
\begin{gathered}
C_{I}^{*} \frac{\partial U_{I}}{\partial Y} \frac{\partial Y}{\partial y}=0 \\
\frac{\partial U_{I}}{\partial Y}=0
\end{gathered}
$$

Y la condición inicial

Cuando $\tau=0$

$$
\begin{gathered}
C_{i}^{*} U_{i}=C_{i 0}(y), \quad i=\eta, I \\
U_{i}=U_{i 0}(Y), \quad i=\eta, I
\end{gathered}
$$




\section{APÉNDICE B-1}

\section{Detalles de la solución del problema de Sturm-Liouville para los dos modelos}

\section{B-1.1 MODELO SIN SALTO}

Ecuaciones diferenciales

$$
\begin{gathered}
\frac{d^{2} \varphi_{\eta}}{d Y^{2}}+\mu_{\eta}^{2} \varphi_{\eta}=0 \text { para } 1<Y<Y_{\eta} \text { donde } \mu_{\eta}^{2}=\alpha_{\eta}^{-1} \lambda^{2} \\
\frac{d^{2} \varphi_{I}}{d Y^{2}}+\mu_{I}^{2} \varphi_{I}=0 \quad \text { para } \quad 0<Y<+1 \quad \text { donde } \mu_{I}^{2}=\alpha_{I}^{-1}\left(\lambda^{2}-\Phi_{I}^{2}\right)
\end{gathered}
$$

Condiciones de frontera

$$
\begin{gathered}
\text { En } Y=Y_{\eta} \quad \varphi_{\eta}=0 \\
\text { En } Y=1 \quad \varphi_{\eta}=\varphi_{I} \\
\text { En } Y=1 \quad \kappa_{\eta} \frac{d \varphi_{\eta}}{d Y}=\frac{d \varphi_{I}}{d Y} \\
\text { En } Y=0 \quad \frac{d \varphi_{I}}{d Y}=0
\end{gathered}
$$

Las soluciones de (B-1.1) y (B-1.2) son:

$$
\begin{aligned}
& \varphi_{\eta}(Y)=A_{\eta} \sin \left(\mu_{\eta} Y\right)+B_{\eta} \cos \left(\mu_{\eta} Y\right) \\
& \varphi_{I}(Y)=A_{I} \sin \left(\mu_{I} Y\right)+B_{I} \cos \left(\mu_{I} Y\right)
\end{aligned}
$$


Con sus respectivas derivadas

$$
\begin{aligned}
& \varphi_{\eta}^{\prime}(Y)=\mu_{\eta}\left[A_{\eta} \cos \left(\mu_{\eta} Y\right)-B_{\eta} \sin \left(\mu_{\eta} Y\right)\right] \\
& \varphi_{I}^{\prime}(Y)=\mu_{I}\left[A_{I} \cos \left(\mu_{I} Y\right)-B_{I} \sin \left(\mu_{I} Y\right)\right]
\end{aligned}
$$

Para determinar el valor de las constantes primero usaremos (B-1.3) y (B-1.6) con (B-1.7) y (B-1.10) respectivamente para obtener

$$
\begin{gathered}
B_{\eta}=-A_{\eta} \frac{\sin \left(\mu_{\eta} Y_{\eta}\right)}{\cos \left(\mu_{\eta} Y_{\eta}\right)} \\
A_{I}=0
\end{gathered}
$$

Así las ecuaciones (B-1.7) y (B-1.8) para $\varphi_{\eta}(Y)$ y $\varphi_{I}(Y)$ toman la siguiente forma

$$
\begin{gathered}
\varphi_{\eta}(Y)=A_{\eta} \frac{\sin \left[\mu_{\eta}\left(Y-Y_{\eta}\right)\right]}{\cos \left(\mu_{\eta} Y_{\eta}\right)} \\
\varphi_{I}(Y)=B_{I} \cos \left(\mu_{I} Y\right)
\end{gathered}
$$

Ahora utilizando las condiciones (B-1.4) y (B-1.5) con (B-1.13) y (B-1.14) dan como resultados

$$
\begin{gathered}
B_{I} \cos \left(\mu_{I}\right)=A_{\eta} \frac{\sin \left[\mu_{\eta}\left(1-Y_{\eta}\right)\right]}{\cos \left(\mu_{\eta} Y_{\eta}\right)} \\
-B_{I} \sin \left(\mu_{I}\right)=\frac{\kappa_{\eta} \mu_{\eta}}{\mu_{I}} A_{\eta} \frac{\cos \left[\mu_{\eta}\left(1-Y_{\eta}\right)\right]}{\cos \left(\mu_{\eta} Y_{\eta}\right)}
\end{gathered}
$$


De la ecuación (B-1.15) se obtiene

$$
B_{I}=A_{\eta} \frac{\sin \left[\mu_{\eta}\left(1-Y_{\eta}\right)\right]}{\cos \left(\mu_{\eta} Y_{\eta}\right) \cos \left(\mu_{I}\right)}
$$

A partir de la combinación de (B-1.15) y (B-1.16) se obtiene la condición de los valores propios

$$
\frac{\kappa_{\eta} \mu_{\eta, n}}{\mu_{I, n}} \cos \left[\mu_{\eta, n}\left(1-Y_{\eta}\right)\right]+\frac{\sin \left(\mu_{I, n}\right)}{\cos \left(\mu_{I, n}\right)} \sin \left[\mu_{\eta, n}\left(1-Y_{\eta}\right)\right]=0
$$

Usando como constante arbitraria $A_{\eta}=1$ en las ecuaciones (B-1.13) y (B-1.14) permiten escribir las funciones propias como:

$$
\begin{gathered}
\varphi_{\eta, n}(Y)=\frac{\sin \left[\mu_{\eta, n}\left(Y-Y_{\eta}\right)\right]}{\cos \left(\mu_{\eta, n} Y_{\eta}\right)} \text { para } 1<Y<Y_{\eta} \\
\varphi_{I, n}(Y)=\frac{\sin \left[\mu_{\eta, n}\left(1-Y_{\eta}\right)\right]}{\cos \left(\mu_{\eta, n} Y_{\eta}\right)} \frac{\cos \left(\mu_{I, n} Y\right)}{\cos \left(\mu_{I, n}\right)} \text { para } 0<Y<1
\end{gathered}
$$

\section{B-1.2 MODELO CON SALTO}

Ecuación diferencial

$$
\frac{d^{2} \varphi_{\eta}^{s}}{d Y^{2}}+\left(\mu_{\eta}^{s}\right)^{2} \varphi_{\eta}^{s}=0 \quad \text { para } 0<Y<Y_{\eta}
$$

Condiciones de frontera

$$
\begin{gathered}
\text { En } Y=Y_{\eta} \quad \varphi_{\eta}^{s}=0 \\
\text { En } Y=0 \quad \kappa_{\eta} \varphi_{\eta}^{s}{ }^{\prime}(0)+\alpha_{I}^{-1}\left[\left(\lambda^{s}\right)^{2}-\Phi_{I}^{2}\right] \varphi_{\eta}^{s}(0)=0
\end{gathered}
$$


La solución de (B-1.21) es:

$$
\varphi_{\eta}^{s}(Y)=A_{\eta} \sin \left(\mu_{\eta}^{s} Y\right)+B_{\eta} \cos \left(\mu_{\eta}^{s} Y\right)
$$

Y su derivada es

$$
\varphi_{\eta}^{s}(Y)=\mu_{\eta}^{s}\left[A_{\eta} \cos \left(\mu_{\eta}^{s} Y\right)-B_{\eta} \sin \left(\mu_{\eta}^{s} Y\right)\right]
$$

Al sustituir la condición de frontera (B-1.22) en (B-1.24) y la sustitución de la constante arbitraria $A_{\eta}=1$ se obtiene la función propia

$$
\begin{gathered}
\varphi_{\eta, n}^{s}(Y)=\frac{\sin \left[\mu_{\eta, n}^{s}\left(Y-Y_{\eta}\right)\right]}{\cos \left(\mu_{\eta, n}^{s} Y_{\eta}\right)} \\
\varphi_{\eta, n}^{s}{ }^{\prime}(Y)=\mu_{\eta, n}^{s} \frac{\cos \left[\mu_{\eta, n}^{s}\left(Y-Y_{\eta}\right)\right]}{\cos \left(\mu_{\eta, n}^{s} Y_{\eta}\right)}
\end{gathered}
$$

El uso de la condición de frontera restante nos llevara a una ecuación para encontrar los valores propios

$$
\kappa_{\eta} \mu_{\eta, n}^{s}+\left(\mu_{I, n}^{s}\right)^{2} \frac{\sin \left[-Y_{\eta} \mu_{\eta, n}^{s}\right]}{\cos \left[-Y_{\eta} \mu_{\eta, n}^{s}\right]}=0
$$




\section{APÉNDICE B-2}

\section{Deducción de la condición de ortogonalidad asociada a las funciones propias para los dos modelos}

\section{B-2.1 MODELO SIN SALTO}

Para la deducción de la condición de ortogonalidad para este modelo se utiliza la identidad de Green. Primero se asigna $w=\varphi_{i, n}$ y $u=\varphi_{i, p}$, después se sustituye en la formula de Green dada por (2.40) para cada fase, resultando:

$$
\begin{gathered}
I_{\eta}=\int_{1}^{Y_{\eta}}\left[\varphi_{\eta, p} \frac{d^{2} \varphi_{\eta, n}}{d Y^{2}}-\varphi_{\eta, n} \frac{d^{2} \varphi_{\eta, p}}{d Y^{2}}\right] d Y=\left[\varphi_{\eta, p} \frac{d \varphi_{\eta, n}}{d Y}-\varphi_{\eta, n} \frac{d \varphi_{\eta, p}}{d Y^{2}}\right]_{1}^{Y_{\eta}} \\
I_{I}=\int_{0}^{1}\left[\varphi_{I, p} \frac{d^{2} \varphi_{I, n}}{d Y^{2}}-\varphi_{I, n} \frac{d^{2} \varphi_{I, p}}{d Y^{2}}\right] d Y=\left[\varphi_{I, p} \frac{d \varphi_{I, n}}{d Y}-\varphi_{I, n} \frac{d \varphi_{I, p}}{d Y^{2}}\right]_{0}^{1}
\end{gathered}
$$

Al sustituir las condiciones de frontera dadas por (B-1.3) y (B-1.6) las ecuaciones (B-2.1) y (B-2.2) se pueden escribir como:

$$
\begin{gathered}
I_{\eta}=\frac{1}{\alpha_{\eta}}\left(\lambda_{p}^{2}-\lambda_{n}^{2}\right) \int_{1}^{Y_{\eta}}\left[\varphi_{\eta, p} \varphi_{\eta, n}\right] d Y=-\varphi_{\eta, p}(1) \varphi_{\eta, n}^{\prime}(1)+\varphi_{\eta, n}(1) \varphi_{\eta, p}^{\prime}(1) \\
I_{I}=\frac{1}{\alpha_{I}}\left(\lambda_{p}^{2}-\lambda_{n}^{2}\right) \int_{0}^{1}\left[\varphi_{I, p} \varphi_{I, n}\right] d Y=\varphi_{I, p}(1) \varphi_{I, n}^{\prime}(1)-\varphi_{I, n}(1) \varphi_{I, p}^{\prime}(1)
\end{gathered}
$$

Al sustituir las condiciones de frontera (B-1.4) y (B-1.5) en (B-2.3) resulta

$$
\kappa_{\eta} I_{\eta}=\frac{\kappa_{\eta}}{\alpha_{\eta}}\left(\lambda_{p}^{2}-\lambda_{n}^{2}\right) \int_{1}^{Y_{\eta}}\left[\varphi_{\eta, p} \varphi_{\eta, n}\right] d Y=-\varphi_{I, p}(1) \varphi_{I, n}^{\prime}(1)+\varphi_{I, n}(1) \varphi_{I, p}^{\prime}(1)
$$

Al sumar las ecuaciones (B-2.4) y (B-2.5) se obtiene la condición de ortogonalidad

$$
\frac{\kappa_{\eta}}{\alpha_{\eta}} \int_{1}^{Y_{\eta}}\left[\varphi_{\eta, p} \varphi_{\eta, n}\right] d Y+\frac{1}{\alpha_{I}} \int_{0}^{1}\left[\varphi_{I, p} \varphi_{I, n}\right] d Y=0, \text { para } p \neq n
$$




\section{B-2.2 MODELO CON SALTO}

Para la deducción de la condición de ortogonalidad para este modelo se utiliza la identidad de Green. Primero se asigna $w=\varphi_{i, n}$ y $u=\varphi_{i, p}$, después se sustituye en la formula de Green dada por (2.40) para la fase $\eta$, resultando:

$$
I_{\eta}=\int_{0}^{Y_{\eta}}\left[\varphi_{\eta, p}^{s} \frac{d^{2} \varphi_{\eta, n}^{s}}{d Y^{2}}-\varphi_{\eta, n} \frac{d^{2} \varphi_{\eta, p}^{s}}{d Y^{2}}\right] d Y=\left[\varphi_{\eta, p}^{s} \frac{d \varphi_{\eta, n}^{s}}{d Y}-\varphi_{\eta, n}^{s} \frac{d \varphi_{\eta, p}^{s}}{d Y^{2}}\right]_{0}^{Y_{\eta}}
$$

Al sustituir la condición de frontera (B-1.22), la ecuación (B-2.7) se pueden escribir como:

$$
I_{\eta}=\frac{1}{\alpha_{\eta}}\left[\left(\lambda_{p}^{s}\right)^{2}-\left(\lambda_{n}^{s}\right)^{2}\right] \int_{0}^{Y_{\eta}}\left[\varphi_{\eta, p}^{s} \varphi_{\eta, n}^{s}\right] d Y=-\varphi_{\eta, p}^{s}(0) \varphi_{\eta, n}^{s}{ }^{\prime}(0)+\varphi_{\eta, n}^{s}(0) \varphi_{\eta, p}^{s}(0)
$$

Después de multiplicar (B-2.8) por $\kappa_{\eta}$, se sustituye la condición de frontera (B-1.23) obteniendo

$$
\kappa_{\eta} I_{\eta}=\frac{\kappa_{\eta}}{\alpha_{\eta}}\left[\left(\lambda_{p}^{s}\right)^{2}-\left(\lambda_{n}^{s}\right)^{2}\right] \int_{0}^{Y_{\eta}}\left[\varphi_{\eta, p}^{s} \varphi_{\eta, n}^{s}\right] d Y=\alpha_{I}^{-1}\left[\left(\lambda_{n}^{s}\right)^{2}-\left(\lambda_{p}^{s}\right)^{2}\right] \varphi_{\eta, p}^{s}(0) \varphi_{\eta, n}^{s}(0)(B
$$

Por último al rearreglar la ecuación se obtiene la condición de ortogonalidad para este modelo

$$
\frac{\kappa_{\eta}}{\alpha_{\eta}} \int_{0}^{Y_{\eta}}\left[\varphi_{\eta, p}^{s} \varphi_{\eta, p}^{s}\right] d Y+\alpha_{I}^{-1} \varphi_{\eta, p}^{s}(0) \varphi_{\eta, n}^{s}(0)=0, \quad \text { para } n \neq p
$$




\section{APÉNDICE B-3}

\section{Evaluación del término $D_{n} \mathbf{y} D_{\eta}^{S}$}

\section{B-3.1 MODELO SIN SALTO}

$$
D_{n}=\sum_{i=1}^{2} \kappa_{i} \alpha_{i}^{-1} \int_{Y_{i-1}}^{Y_{i}} \varphi_{i, n}^{s} d Y=\frac{1}{\alpha_{I}} \int_{0}^{1} \varphi_{I, n}^{2} d Y+\frac{\kappa_{\eta}}{\alpha_{\eta}} \int_{1}^{Y_{\eta}} \varphi_{\eta, n}^{2} d Y
$$

Para resolver las integrales en (B-3.1) se utilizaron las definiciones de las funciones propias (B-1.19) y (B-1.20), resultando

$$
\begin{gathered}
\int_{0}^{1} \varphi_{I, n}^{2} d Y=\frac{\sin ^{2}\left[\mu_{\eta, n}\left(1-Y_{\eta}\right)\right]}{\cos ^{2}\left(\mu_{\eta, n} Y_{\eta}\right) \cos ^{2}\left(\mu_{I, n}\right)} \int_{0}^{1} \cos ^{2}\left(\mu_{I, n} Y\right) d Y=\frac{\sin ^{2}\left[\mu_{\eta, n}\left(1-Y_{\eta}\right)\right]}{\cos ^{2}\left(\mu_{\eta, n} Y_{\eta}\right) \cos ^{2}\left(\mu_{I, n}\right)}\left[\frac{1}{2}+\frac{\operatorname{sen}\left(2 \mu_{I, n}\right)}{4 \mu_{I, n}}\right] \\
\int_{1}^{Y_{\eta}} \varphi_{\eta, n}^{2} d Y=\frac{1}{\cos ^{2}\left(\mu_{\eta, n} Y_{\eta}\right)} \int_{1}^{Y_{\eta}} \sin ^{2}\left[\mu_{\eta, n}\left(Y-Y_{\eta}\right)\right] d Y=\frac{\operatorname{sen}\left[2 \mu_{\eta, n}\left(1-Y_{\eta}\right)\right]-2 \mu_{\eta, n}\left(1-Y_{\eta}\right)}{4 \mu_{\eta, n} \cos ^{2}\left(\mu_{\eta, n} Y_{\eta}\right)} \\
D_{n}=\frac{\sin ^{2}\left[\mu_{\eta, n}\left(1-Y_{\eta}\right)\right]}{\cos ^{2}\left(\mu_{\eta, n} Y_{\eta}\right) \cos ^{2}\left(\mu_{I, n}\right)}\left[\frac{2 \mu_{I, n}+\operatorname{sen}\left(2 \mu_{I, n}\right)}{4 \mu_{I, n} \alpha_{I}}\right]+\left(\frac{\kappa_{\eta}}{\alpha_{\eta}}\right) \frac{\operatorname{sen}\left[2 \mu_{\eta, n}\left(1-Y_{\eta}\right)\right]-2 \mu_{\eta, n}\left(1-Y_{\eta}\right)}{4 \mu_{\eta, n} \cos ^{2}\left(\mu_{\eta, n} Y_{\eta}\right)}
\end{gathered}
$$

\section{B-3.2 MODELO CON SALTO}

$$
D_{n}^{s}=\kappa_{\eta} \alpha_{\eta}^{-1} \int_{0}^{Y_{\eta}}\left(\varphi_{\eta, n}^{s}\right)^{2} d Y+\alpha_{I}^{-1}\left(\varphi_{\eta, n}^{s}\right)^{2}(0)
$$


Para resolver las integrales en (B-3.5) se utilizo la definición de la función propia (B-1.26), resultando

$$
\begin{gathered}
\int_{0}^{Y_{\eta}}\left(\varphi_{\eta, n}^{s}\right)^{2} d Y=\frac{1}{\cos ^{2}\left(\mu_{\eta, n}^{s} Y_{\eta}\right)} \int_{0}^{Y_{\eta}} \sin ^{2}\left[\mu_{\eta, n}^{s}\left(Y-Y_{\eta}\right)\right] d Y=\frac{1}{\cos ^{2}\left(\mu_{\eta, n}^{s} Y_{\eta}\right)}\left[\frac{\operatorname{sen}\left[-2 \mu_{\eta, n}^{s} Y_{\eta}\right]}{4 \mu_{\eta, n}^{s}}+\frac{Y_{\eta}}{2}\right] \\
\left(\varphi_{\eta, n}^{s}\right)^{2}(0)=\frac{\sin ^{2}\left[-\mu_{\eta, n}^{s} Y_{\eta}\right]}{\cos ^{2}\left(\mu_{\eta, n}^{s} Y_{\eta}\right)} \\
D_{n}^{s}=\frac{\kappa_{\eta}}{\alpha_{\eta} \cos ^{2}\left(\mu_{\eta, n}^{s} Y_{\eta}\right)}\left[\frac{\operatorname{sen}\left[-2 \mu_{\eta, n}^{s} Y_{\eta}\right]+2 \mu_{\eta, n}^{s} Y_{\eta}}{4 \mu_{\eta, n}^{s}}\right]+\frac{\sin ^{2}\left[-\mu_{\eta, n}^{s} Y_{\eta}\right]}{\alpha_{I} \cos ^{2}\left(\mu_{\eta, n}^{s} Y_{\eta}\right)}
\end{gathered}
$$




\section{APÉNDICE B-4}

\section{Evaluación de $C_{n, i}(\tau)$ y $C_{n}^{s}(\tau)$}

\section{B-4.1 MODELO SIN SALTO}

Fase $\eta$

$$
C_{n, n}(\tau)=\frac{1}{D_{n}} e^{-\lambda_{n}^{2} \tau} \int_{0}^{\tau} e^{\lambda_{n}^{2} \zeta} I_{n}(\zeta) d \zeta
$$

\section{Caso I-a}

$$
\begin{gathered}
F_{\eta}(\tau)=U_{\eta}^{1} \\
I_{n}(\tau)=-\kappa_{\eta} U_{\eta}^{1} \frac{\mu_{\eta, n}}{\cos \left(\mu_{\eta, n} Y_{\eta}\right)}
\end{gathered}
$$

Sustituyendo (B-4.3) en (B-4.1) resulta

$$
\begin{gathered}
C_{n, \eta}(\tau)=-\frac{1}{D_{n}} e^{-\left(\alpha_{\eta} \mu_{\eta, n}^{2}\right) \tau} \frac{\kappa_{\eta} U_{\eta}^{1} \mu_{\eta, n}}{\cos \left(\mu_{\eta, n} Y_{\eta}\right)} \int_{0}^{\tau} e^{\left(\alpha_{\eta} \mu_{\eta, n}^{2}\right) \zeta} d \zeta \\
C_{n, \eta}(\tau)=\frac{1}{D_{n}} \frac{\kappa_{\eta} U_{\eta}^{1}}{\cos \left(\mu_{\eta, n} Y_{\eta}\right) \alpha_{\eta} \mu_{\eta, n}}\left[e^{-\left(\alpha_{\eta} \mu_{\eta, n}^{2}\right) \tau}-1\right]
\end{gathered}
$$

\section{Caso I-b}

$$
\begin{gathered}
F_{\eta}(\tau)=U_{\eta}^{1}-\sigma_{\eta} e^{-\beta_{\eta} \tau} \\
I_{n}(\tau)=-\frac{\kappa_{\eta} \mu_{\eta, n}\left(U_{\eta}^{1}-\sigma_{\eta} e^{-\beta_{\eta} \tau}\right)}{\cos \left(\mu_{\eta, n} Y_{\eta}\right)}
\end{gathered}
$$

Sustituyendo (B-4.7) en (B-4.1) resulta 


$$
\begin{gathered}
C_{n, \eta}(\tau)=R_{\eta} e^{-\left(\alpha_{\eta} \mu_{\eta, n}^{2}\right) \tau}\left[U_{\eta}^{1} \int_{0}^{\tau} e^{\left(\alpha_{\eta} \mu_{\eta, n}^{2}\right) \zeta} d \zeta-\sigma_{\eta} \int_{0}^{\tau} e^{\left(\alpha_{\eta} \mu_{\eta, n}^{2}-\beta_{\eta}\right) \zeta} d \zeta\right] \\
C_{n, \eta}(\tau)=\frac{R_{\eta} U_{\eta}^{1}}{\left(\alpha_{\eta} \mu_{\eta, n}^{2}\right)}\left[1-e^{-\left(\alpha_{\eta} \mu_{\eta, n}^{2}\right) \tau}\right]-\frac{R_{\eta} \sigma_{\eta}}{\left(\alpha_{\eta} \mu_{\eta, n}^{2}-\beta_{\eta}\right)}\left[e^{-\beta_{\eta} \tau}-e^{-\left(\alpha_{\eta} \mu_{\eta, n}^{2}\right) \tau}\right]
\end{gathered}
$$

Fase I

$$
C_{n, I}(\tau)=\frac{1}{D_{n}} e^{-\left(\alpha_{I} \mu_{I, n}^{2}+\Phi_{I}^{2}\right) \tau} \int_{0}^{\tau} e^{\left(\alpha_{I} \mu_{I, n}^{2}+\Phi_{I}^{2}\right) \zeta} I_{n}(\zeta) d \zeta
$$

\section{Caso I-a}

La función $I_{n}(\tau)$ ya se definió anteriormente por la ecuación (B-4.3), que al sustituir en (B-4.10) y resolviendo la integral, resulta

$$
\begin{gathered}
C_{n, I}(\tau)=-\frac{1}{D_{n}} \frac{\kappa_{\eta} U_{\eta}^{1} \mu_{\eta, n}}{\cos \left(\mu_{\eta, n} Y_{\eta}\right)} e^{-\left(\alpha_{I} \mu_{I, n}^{2}+\Phi_{I}^{2}\right) \tau} \int_{0}^{\tau} e^{\left(\alpha_{I} \mu_{I, n}^{2}+\Phi_{I}^{2}\right) \zeta} d \zeta \\
C_{n, I}(\tau)=\frac{1}{D_{n}} \frac{\kappa_{\eta} U_{\eta}^{1} \mu_{\eta, n}}{\cos \left(\mu_{\eta, n} Y_{\eta}\right)} \frac{1}{\left(\alpha_{I} \mu_{I, n}^{2}+\Phi_{I}^{2}\right)}\left[e^{-\left(\alpha_{I} \mu_{I, n}^{2}+\Phi_{I}^{2}\right) \tau}-1\right]
\end{gathered}
$$

\section{Caso I-b}

La función $I_{n}(\tau)$ ya se definió anteriormente por la ecuación (B-4.7), que al sustituir en (B-4.10) y resolviendo la integral, resulta

$$
C_{n, I}(\tau)=-\frac{1}{D_{n}} \frac{\kappa_{\eta} \mu_{\eta, n}}{\cos \left(\mu_{\eta, n} Y_{\eta}\right)} e^{-\left(\alpha_{I} \mu_{I, n}^{2}+\Phi_{I}^{2}\right) \tau}\left[\begin{array}{l}
U_{\eta}^{1} \int_{0}^{\tau} e^{\left(\alpha_{I} \mu_{I, n}^{2}+\Phi_{I}^{2}\right) \zeta} d \zeta \\
-\sigma_{\eta} \int_{0}^{\tau} e^{\left(\alpha_{I} \mu_{I, n}^{2}+\Phi_{I}^{2}-\beta_{\eta}\right) \zeta} d \zeta
\end{array}\right]
$$




$$
\begin{aligned}
& C_{n, I}(\tau)=\frac{R_{\eta} U_{\eta}^{1}}{\left(\alpha_{I} \mu_{I, n}^{2}+\Phi_{I}^{2}\right)}\left[1-e^{-\left(\alpha_{I} \mu_{I, n}^{2}+\Phi_{I}^{2}\right) \tau}\right] \\
&-\frac{R_{\eta} \sigma_{\eta}}{\left(\alpha_{I} \mu_{I, n}^{2}+\Phi_{I}^{2}-\beta_{\eta}\right)}\left[e^{-\beta_{\eta} \tau}-e^{-\left(\alpha_{I} \mu_{I, n}^{2}+\Phi_{I}^{2}\right) \tau}\right]
\end{aligned}
$$

\section{B-4.2 MODELOS CON SALTO}

Fase $\eta$

$$
C_{n}^{s}(\tau)=\frac{1}{D_{n}^{s}} e^{-\left(\mu_{n}^{s}\right)^{2} \alpha_{\eta} \tau} \int_{0}^{\tau} e^{\left(\mu_{n}^{s}\right)^{2} \alpha_{\eta} \zeta} I_{n}^{s}(\zeta) d \zeta
$$

\section{Caso I-a}

$$
\begin{gathered}
F_{\eta}(\tau)=U_{\eta}^{1} \\
I_{n}^{s}(\tau)=-\kappa_{\eta} U_{\eta}^{1} \frac{\mu_{\eta, n}^{s}}{\cos \left(\mu_{\eta, n}^{s} Y_{\eta}\right)}
\end{gathered}
$$

Sustituyendo (B-4.17) en (B-4.15) resulta

$$
\begin{gathered}
C_{n}^{s}(\tau)=-\frac{1}{D_{n}^{s}} e^{-\left[\alpha_{\eta}\left(\mu_{\eta, n}^{s}\right)^{2}\right] \tau} \frac{\kappa_{\eta} U_{\eta}^{1} \mu_{\eta, n}^{s}}{\cos \left(\mu_{\eta, n}^{s} Y_{\eta}\right)} \int_{0}^{\tau} e^{\left[\alpha_{\eta}\left(\mu_{\eta, n}^{s}\right)^{2}\right] \zeta} d \zeta \\
C_{n}^{s}(\tau)=\frac{1}{D_{n}^{s}} \frac{\kappa_{\eta} U_{\eta}^{1}}{\cos \left(\mu_{\eta, n}^{s} Y_{\eta}\right) \alpha_{\eta} \mu_{\eta, n}^{s}}\left[e^{-\left[\alpha_{\eta}\left(\mu_{\eta, n}^{s}\right)^{2}\right] \tau}-1\right]
\end{gathered}
$$

\section{Caso I-b}

$$
\begin{gathered}
F_{\eta}(\tau)=U_{\eta}^{1}-\sigma_{\eta} e^{-\beta_{\eta} \tau} \\
I_{n}^{s}(\tau)=-\frac{\kappa_{\eta} \mu_{\eta, n}^{s}\left(U_{\eta}^{1}-\sigma_{\eta} e^{-\beta_{\eta} \tau}\right)}{\cos \left(\mu_{\eta, n}^{s} Y_{\eta}\right)}
\end{gathered}
$$


Sustituyendo (B-4.21) en (B-4.15) resulta

$$
\begin{gathered}
C_{n}^{s}(\tau)=R_{\eta} e^{-\left[\alpha_{\eta}\left(\mu_{\eta, n}^{s}\right)^{2}\right] \tau}\left[U_{\eta}^{1} \int_{0}^{\tau} e^{\left[\alpha_{\eta}\left(\mu_{\eta, n}^{s}\right)^{2}\right] \zeta} d \zeta-\sigma_{\eta} \int_{0}^{\tau} e^{\left[\alpha_{\eta}\left(\mu_{\eta, n}^{s}\right)^{2}-\beta_{\eta}\right] \zeta} d \zeta\right] \\
C_{n}^{s}(\tau)=\frac{R_{\eta} U_{\eta}^{1}}{\left[\alpha_{\eta}\left(\mu_{\eta, n}^{s}\right)^{2}\right.}\left[1-e^{-\left[\alpha_{\eta}\left(\mu_{\eta, n}^{s}\right)^{2}\right] \tau}\right]-\frac{R_{\eta} \sigma_{\eta}}{\left[\alpha_{\eta}\left(\mu_{\eta, n}^{s}\right)^{2}-\beta_{\eta}\right]}\left[e^{-\beta_{\eta} \tau}-e^{-\left[\alpha_{\eta}\left(\mu_{\eta, n}^{s}\right)^{2}\right] \tau}\right](\mathrm{B}-4.23) \\
\end{gathered}
$$




\section{APÉNDICE C}

\section{DIAGRAMAS DE FLUJO}

Figura C.1 Diagrama de Flujo para evaluar la solución analítica del MSS.

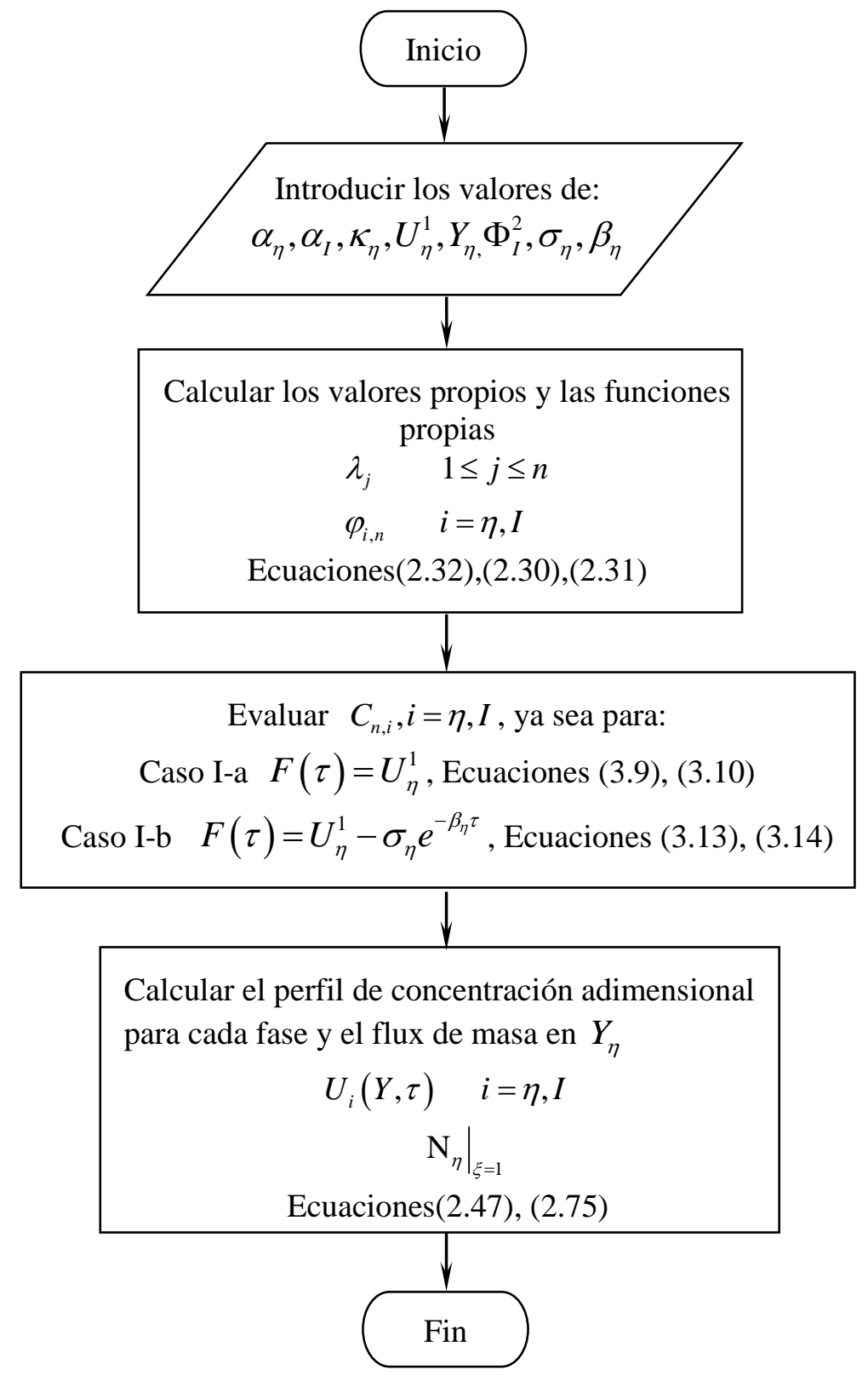


Figura C.2 Diagrama de Flujo para evaluar la solución analítica del MCS.

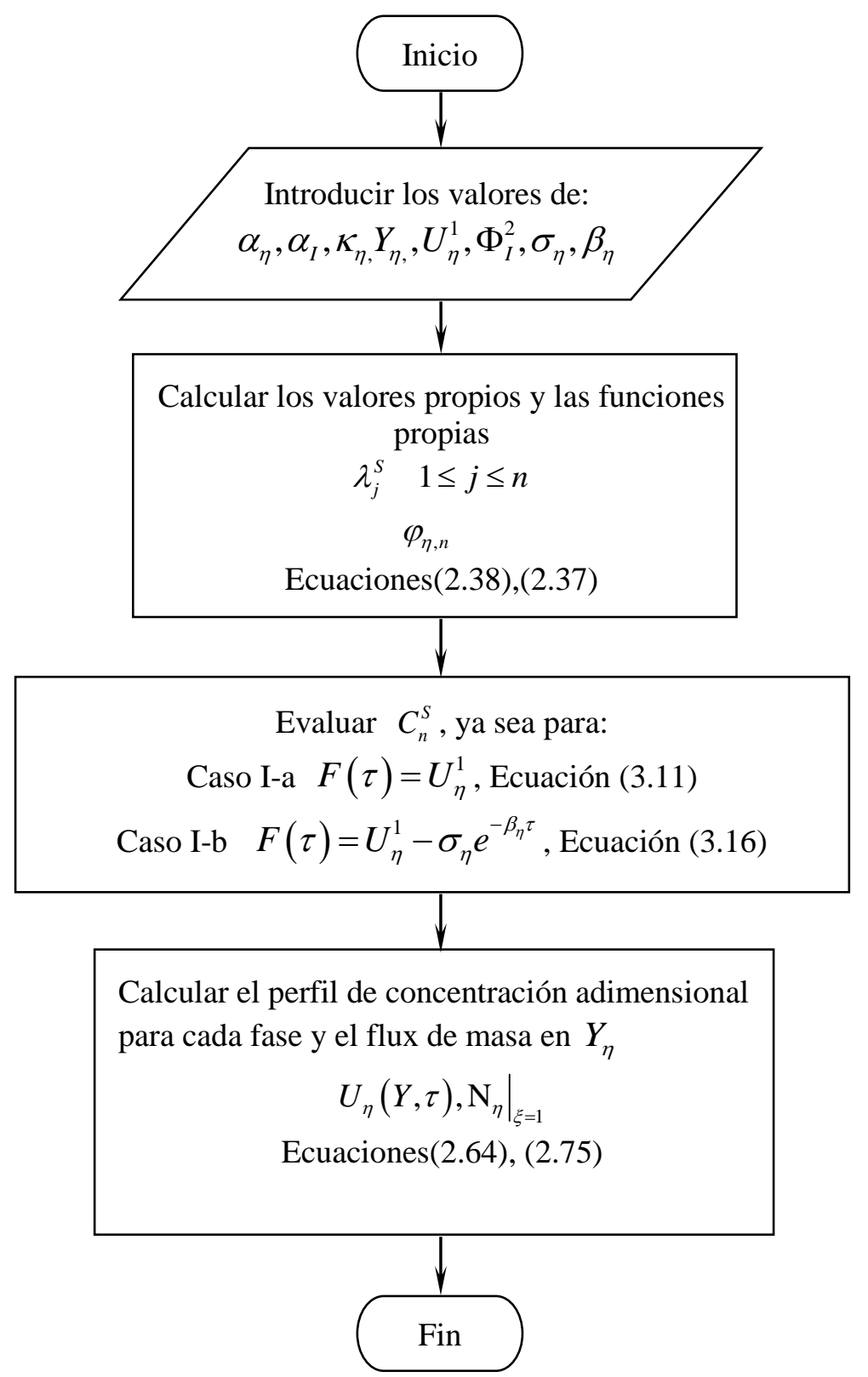




\section{Discretizacion de las EDP}

El sistema de dos fases con y sin salto que contemplan una reacción de segundo orden en la fase $I$, dada por las ecuaciones, se consideraron como ejemplo, para describir a detalle la solución numérica en estado estacionario (Método de Newton Raphson) y en estado transitorio (Método de líneas).

\section{MSS}

Primero se requiere reemplazar las derivadas espaciales con una aproximación algebraica, en este caso se utilizo diferencias finitas, de manera que en base a la siguiente nomenclatura se discretizan las ecuaciones para cada fase y sus respectivas condiciones de frontera:

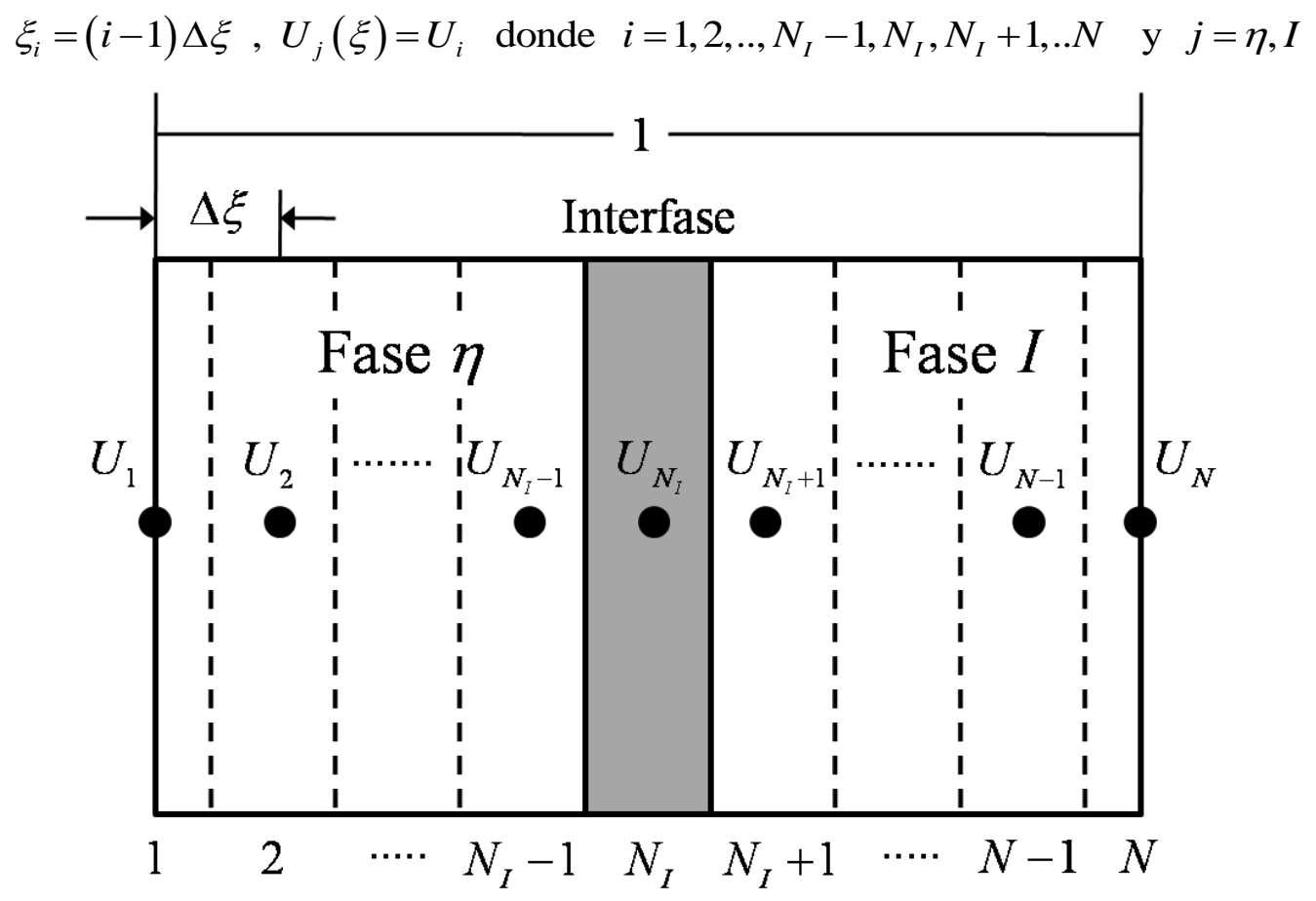

Nodos

Figura C.3 Esquema de la celda computacional utilizada para la solución del sistema de dos fases sin salto. 
Cabe señalar que $N_{I}$ representa el número de incrementos utilizados en la fase $I$ y $N$ el número total de incrementos requeridos para las dos fases, como se muestra en la figura B1, donde $\xi$ representa una nueva variable adimensional en base a la longitud característica de la fase $\eta$ definida como $\xi=Y / Y_{\eta}$.

Fase $\eta$

$$
\begin{gathered}
\frac{\partial U_{\eta}}{\partial \tau}=\left.\delta_{\eta} \frac{\partial^{2} U_{\eta}}{\partial \xi^{2}}\right|_{i}, \quad N_{\mathrm{I}}+1<i<N-1 \\
\frac{\partial U_{i}}{\partial \tau}=R_{\eta}\left(U_{i+1}-2 U_{i}+U_{i-1}\right), \quad N_{\mathrm{I}}+1<i<N-1
\end{gathered}
$$

Donde:

$$
R^{\eta}=\frac{\delta_{\eta}}{\left[(\Delta \xi)_{\eta}\right]^{2}}=\frac{\alpha_{\eta}}{Y_{\eta}^{2}\left[(\Delta \xi)_{\eta}\right]^{2}}
$$

Sujeta a las siguientes condiciones de frontera:

$$
\begin{array}{cc}
\xi=1 & U_{\eta}=\left.F(\tau)\right|_{i} \\
\xi=\frac{1}{Y_{\eta}} & \kappa_{\eta} \frac{\partial U_{\eta}}{\partial \xi}=\frac{\partial U_{\mathrm{I}}}{\partial \xi}
\end{array}
$$

De la condición de frontera (C.5) se obtiene para el caso en que la funcionalidad del tiempo sea una constante $U_{\eta}^{1}=1$

$$
i=N \quad U_{N}=U_{\eta}^{1}
$$

Discretizando la ecuación (C.6), con base a la nomenclatura de diferencias finitas para la primera derivada hacia adelante y atrás, se obtiene

$$
\begin{gathered}
i=N_{I},\left.\quad \kappa_{\eta} \frac{\partial U_{\eta}}{\partial \xi}\right|_{i}=\left.\frac{\partial U_{\mathrm{I}}}{\partial \xi}\right|_{i} \\
i=N_{\mathrm{I}}, \quad U_{N_{I}}=\frac{U_{N_{I}+1}+r U_{N_{I}-1}}{(r+1)}
\end{gathered}
$$

Donde 


$$
r=\frac{(\Delta \xi)_{\eta}}{\kappa_{\eta}(\Delta \xi)_{I}}
$$

Fase I

$$
\begin{gathered}
\frac{\partial U_{\mathrm{I}}}{\partial \tau}=\left.\delta_{\mathrm{I}} \frac{\partial^{2} U_{\mathrm{I}}}{\partial \xi^{2}}\right|_{i}-\left.\Phi_{\mathrm{I}}^{2} U_{\mathrm{I}}^{2}\right|_{i}, \quad 2<i<N_{\mathrm{I}}-1 \\
\frac{\partial U_{i}}{\partial \tau}=R^{I}\left(U_{i+1}-2 U_{i}+U_{i-1}\right)-\Phi_{\mathrm{I}}^{2} U_{i}^{2}, \quad 2<i<N_{\mathrm{I}}-1
\end{gathered}
$$

Donde

$$
R^{I}=\frac{\delta_{I}}{\left[(\Delta \xi)_{I}\right]^{2}}=\frac{\alpha_{I}}{Y_{\eta}^{2}\left[(\Delta \xi)_{I}\right]^{2}}
$$

Sujeta a las siguientes condiciones de frontera:

$$
\begin{array}{ll}
\xi=\frac{1}{Y_{\eta}}, & U_{\eta}=U_{I} \\
\xi=0, & \frac{\partial U_{I}}{\partial \xi}=0
\end{array}
$$

De la condición de frontera (C.14) se obtiene:

$$
i=N_{\mathrm{I}}, \quad U_{N_{I}}=U_{N_{I}}
$$

Discretizando la ecuación (C.15) resulta

$$
\begin{array}{ll}
i=1, & \left.\frac{\partial U_{I}}{\partial \xi}\right|_{i}=0 \\
i=1, & U_{2}=U_{1}
\end{array}
$$

El flux de masa se evalúa de la siguiente manera

$$
\left.\mathrm{N}_{\eta}\right|_{\xi=1}=-\left.\frac{\alpha_{\eta}}{Y_{\eta}} \frac{d U_{\eta}}{d \xi}\right|_{\xi=1}=\frac{\alpha_{\eta}}{Y_{\eta}}\left(\frac{U_{N}-U_{N-1}}{\Delta \xi}\right)
$$


Figura C.4 Diagrama de Flujo para evaluar la solución numérica en estado estacionario del MSS.

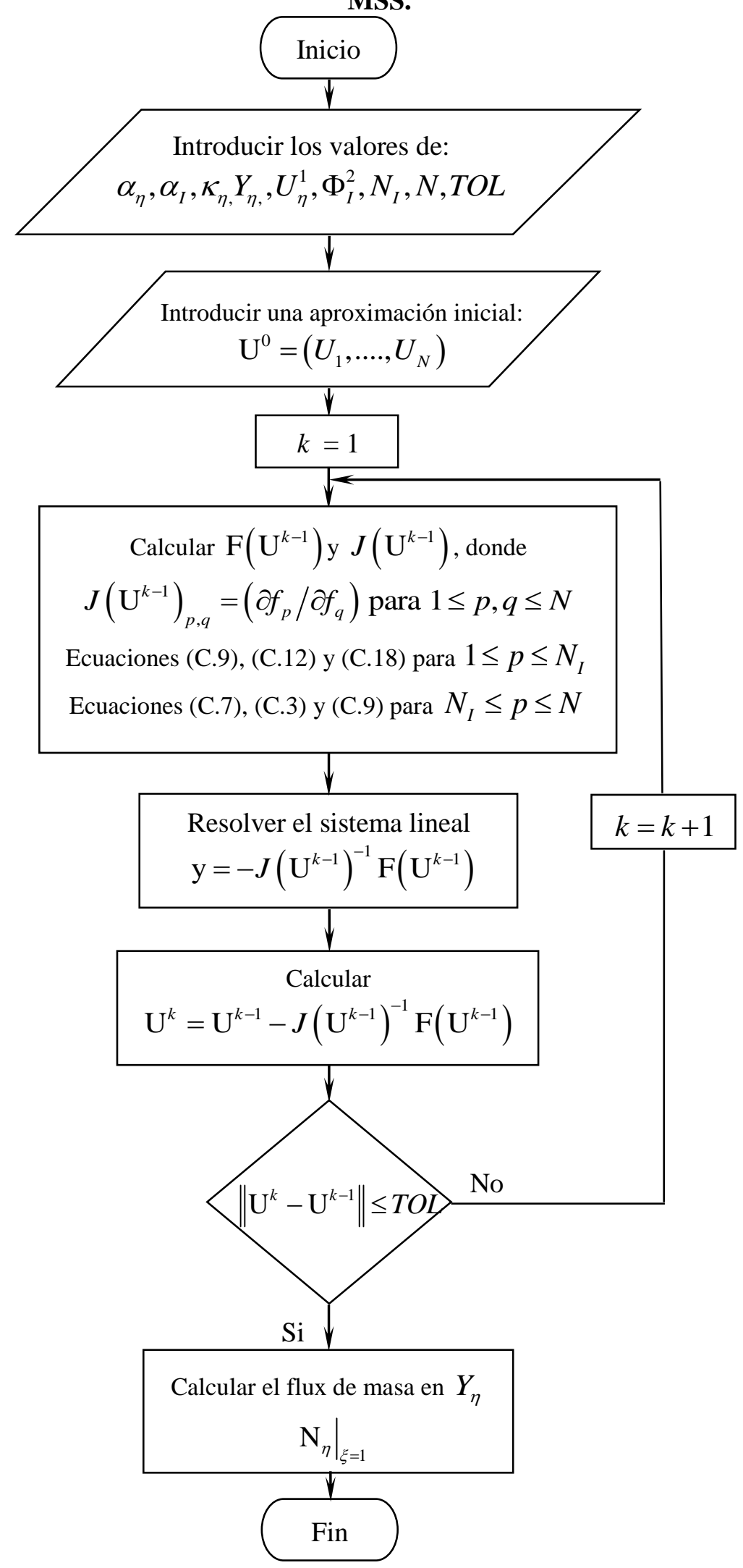


Figura C.5 Diagrama de Flujo para evaluar la solución numérica en estado transitorio del MSS.

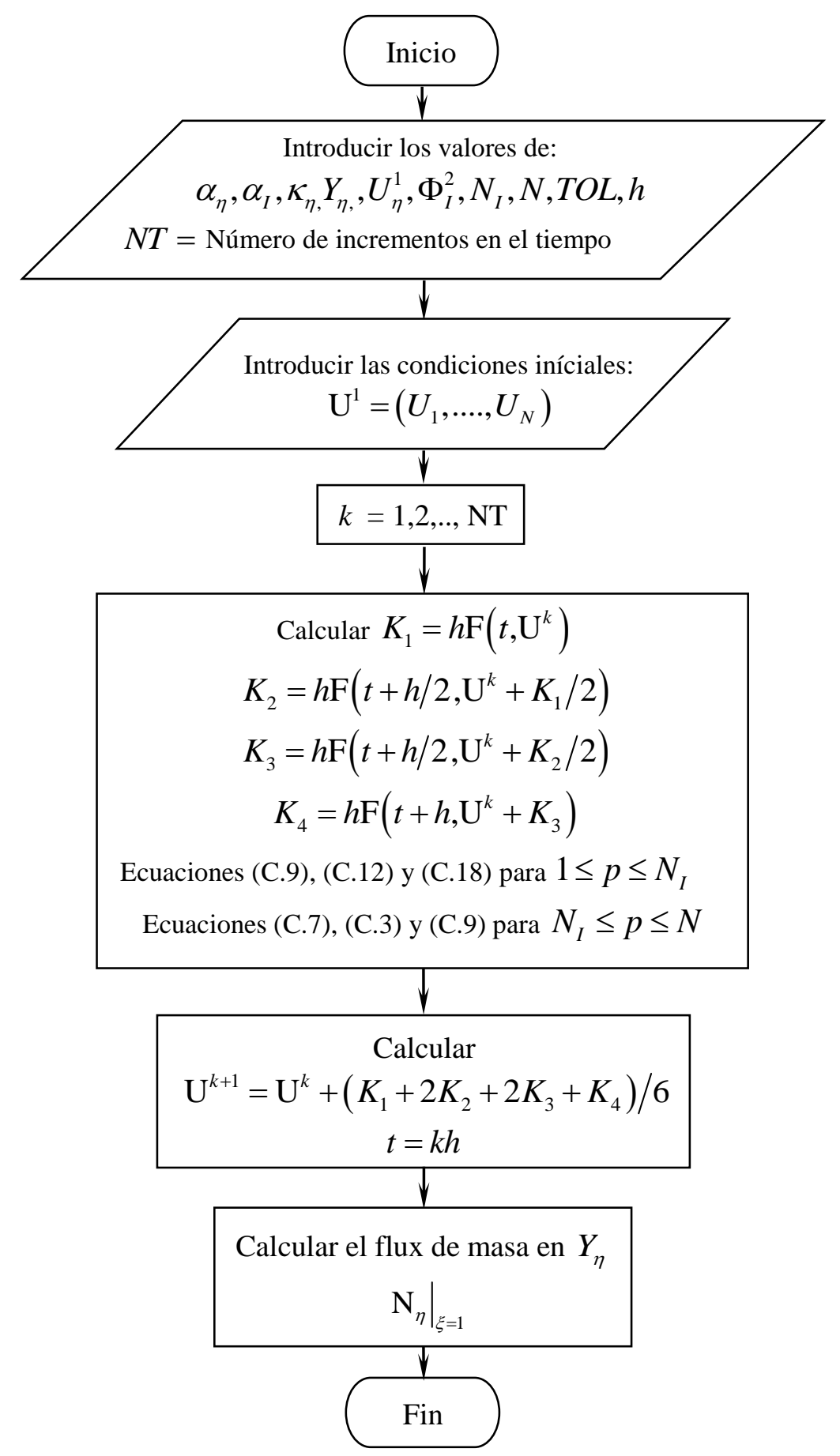


El sistema aproximado consta de $N-3$ EDOs que dependen únicamente de la variable independiente $\tau$ y tres ecuaciones algebraicas que representan las condiciones de frontera. Con base a lo anterior se muestran los diagramas de flujo para el Método de Newton Raphson y el Método de líneas, donde este ultimo incluye el método de Runge Kutta de cuarto orden para la integración del sistema de ecuaciones diferenciales ordinarias.

\section{MCS}

De manera similar que el MSS se requiere reemplazar las derivadas espaciales con una aproximación algebraica, utilizando diferencias finitas, de manera que en base a la siguiente nomenclatura se discretizan las ecuaciones para cada fase y sus respectivas condiciones de frontera:

$$
\xi_{i}=(i-1) \Delta \xi, U_{j}(\xi)=U_{i} \text { donde } i=1,2, \ldots, N \quad \text { y } j=\eta, I
$$

Cabe señalar que $N$ representa el número total de incrementos requeridos para la fase $\eta$. La ecuación discretizada para la fase $\eta$ es similar a la dada por (C.3), solo que el rango de los incrementos es $2<i<N-1$. Además la condición de frontera en $Y=Y_{\eta}$ permanece sin cambios.

La condición de frontera desarrollada para este caso es:

$$
\xi=0 \quad \frac{\partial U_{\eta}}{\partial \tau}=\left.\zeta_{\mathrm{I}} \frac{\partial U_{\eta}}{\partial \xi}\right|_{\xi=0}-\Phi_{\mathrm{I}}^{2} U_{\eta}
$$

Donde

$$
\zeta_{\mathrm{I}}=\frac{\alpha_{\mathrm{I}} \kappa_{\eta}}{Y_{\eta}}
$$

Discretizando la ecuación de la condición de frontera (C.21) se obtiene

$$
i=1, \quad \frac{\partial U_{1}}{\partial \tau}=\frac{\zeta_{\mathrm{I}}}{\Delta \xi}\left(U_{2}-U_{1}\right)-\Phi_{\mathrm{I}}^{2} U_{1}^{2}
$$

El sistema aproximado consta de $N-2$ EDOs que dependen únicamente de la variable independiente $\tau$ y dos ecuaciones algebraicas que representan las condiciones de frontera. 
Figura C.6 Diagrama de Flujo para evaluar la solución numérica en estado estacionario del MCS.

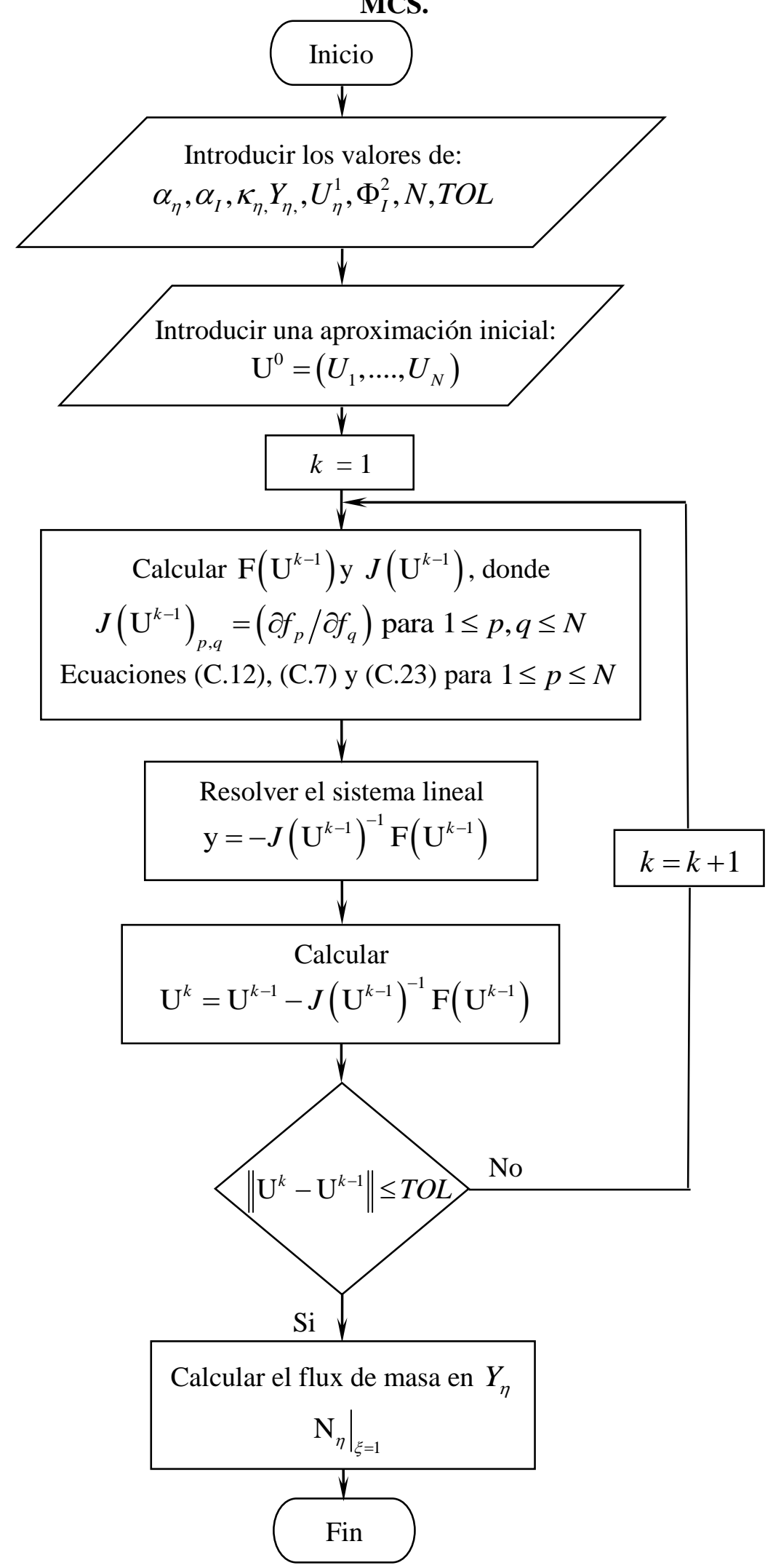


Figura C.7 Diagrama de Flujo para evaluar la solución numérica en estado transitorio del MCS.

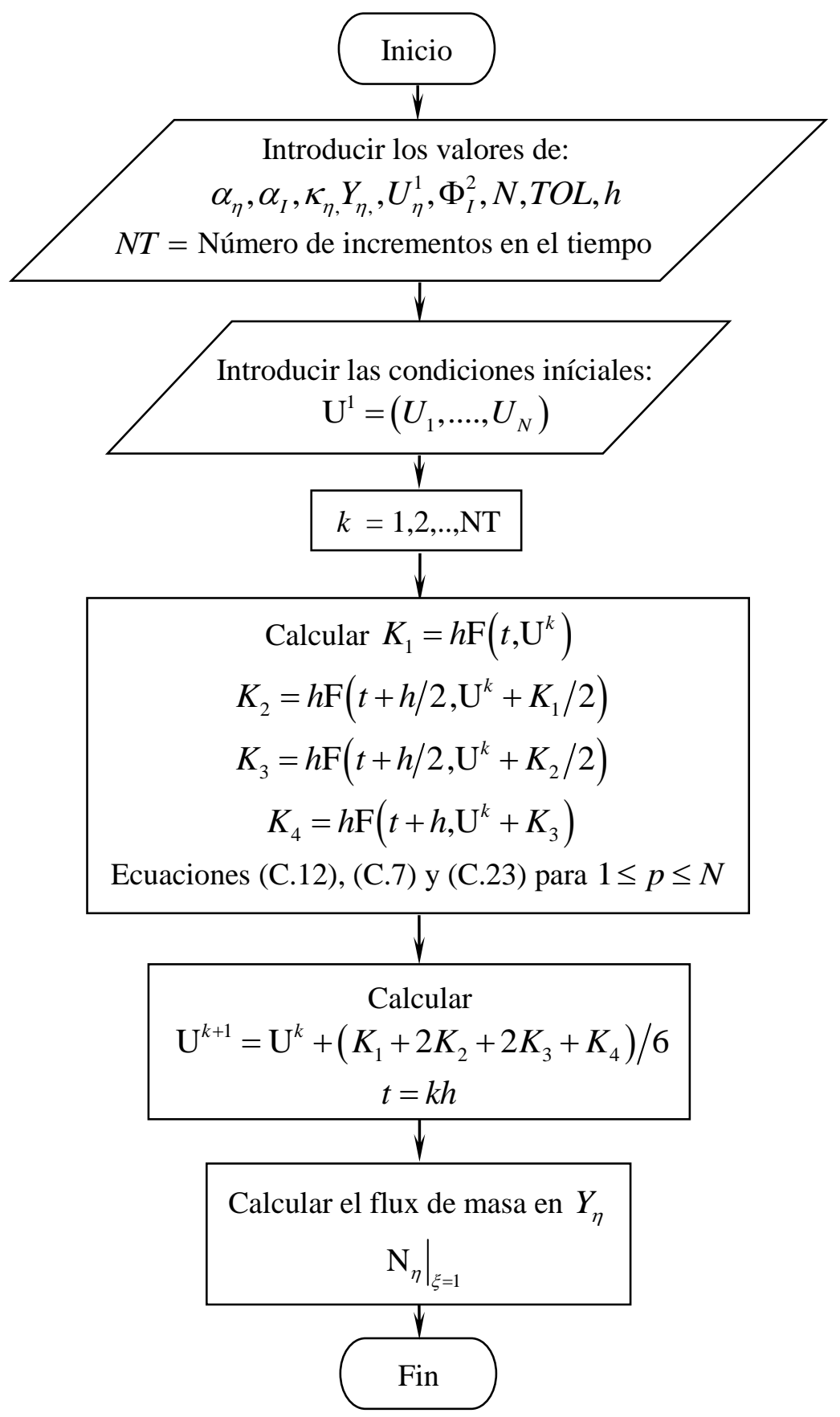




\section{APÉNDICE D}

\section{Búsqueda de los valores propios para el problema lineal}

Un primer paso para evaluar la solución analítica de los modelos es encontrar los valores propios asociados, para lo cual se resuelven las ecuaciones. (2.32) y (2.38). En la Figura C.1 se muestran los valores propios para el MSS y en la Figura C.2 los valores propios correspondientes al MCS, para diferentes valores del módulo de Thiele, manteniendo constante una $Y_{\eta}=5.0$. Para la determinación de estas raíces se utilizó el método de Newton. Es importante mencionar que dado que los valores propios $\lambda_{n}$ son una colección infinita, la solución exacta se obtendría solamente con el conjunto completo de esta colección, sin embargo para propósitos de evaluación se toma un número determinado de valores propios tales que las soluciones tengan convergencia.

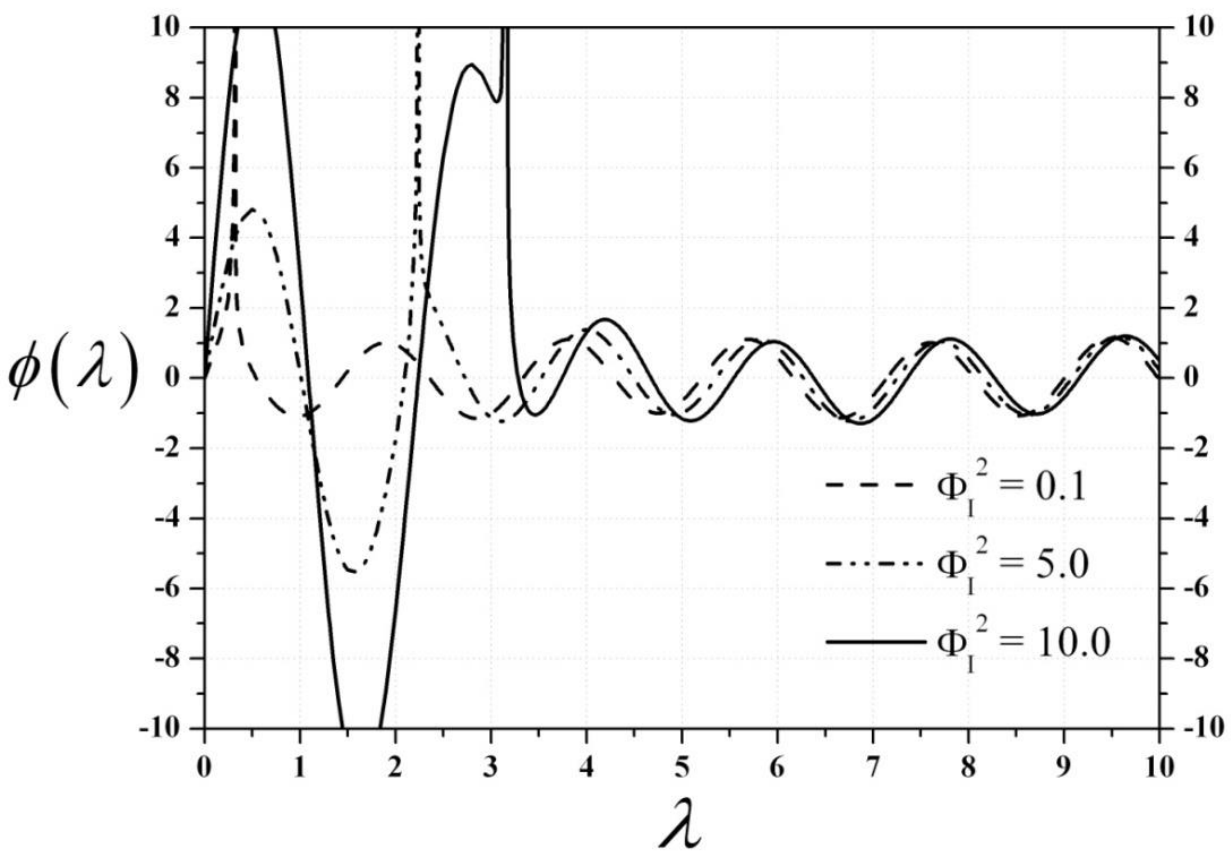

Figura D.1 Evolución de la función que determina los valores propios para el MSS, para tres valores del $\Phi_{I}^{2}$, manteniendo $Y_{\eta}=5$. 


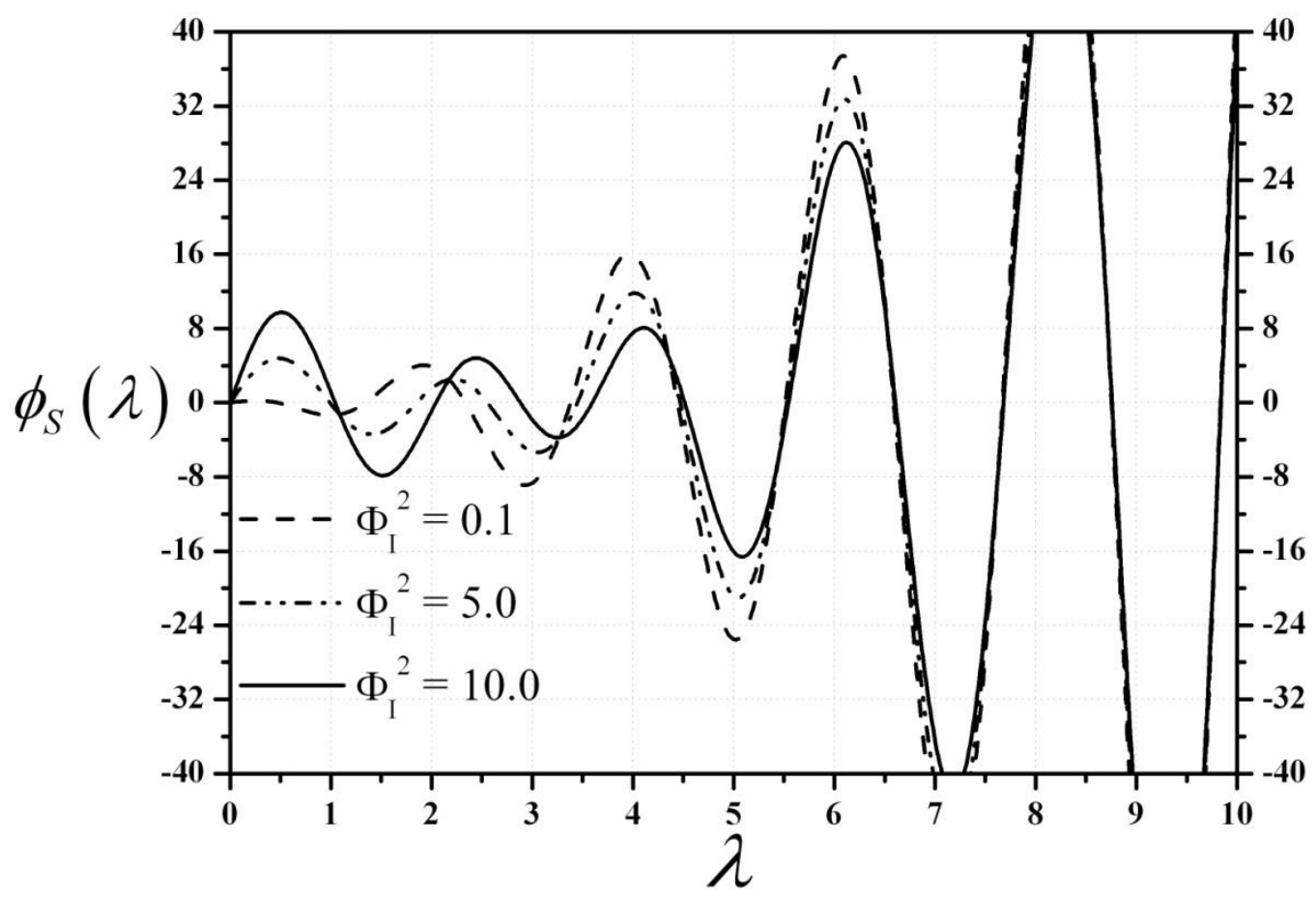

Figura D.2 Evolución de la función que determina los valores propios para el MCS, para tres valores del $\Phi_{\mathrm{I}}^{2}$, manteniendo $Y_{\eta}=5$. 


\section{APÉNDICE E}

\section{Deducción de la solución compuesta mediante el método de superposición}

La solución analítica se puede expresar de forma general de la siguiente manera

$$
U_{i}(Y, \tau)=\sum_{n=1}^{\infty} \varphi_{i, n}(Y) C_{n, i}(\tau) \quad, \quad i=\eta, I
$$

De manera similar que en el método de superposición, se propone descomponer el problema transitorio en dos partes, en este caso el término dependiente del tiempo se define como:

$$
C_{n, i}(\tau)=C_{n, i}^{T}(\tau)+C_{n, i}^{S}
$$

Sustituyendo (E.2) en (E.1) se obtiene

$$
\begin{gathered}
U_{i}(Y, \tau)=\sum_{n=1}^{\infty} \varphi_{i, n}(Y)\left[C_{n, i}^{T}(\tau)+C_{n, i}^{S}\right], \quad i=\eta, I \\
U_{i}(Y, \tau)=\sum_{n=1}^{\infty}\left[\varphi_{i, n}(Y) C_{n, i}^{T}(\tau)+\varphi_{i, n}(Y) C_{n, i}^{S}\right] \quad, \quad i=\eta, I
\end{gathered}
$$

La ecuación (E.4) también se puede escribir como:

$$
U_{i}(Y, \tau)=\sum_{n=1}^{\infty} \varphi_{i, n}(Y) C_{n, i}^{T}(\tau)+\sum_{n=1}^{\infty} \varphi_{i, n}(Y) C_{n, i}^{S} \quad, \quad i=\eta, I
$$

Por otro lado a partir de la ecuación (E.1) se puede obtener la solución en estado estacionario cuando $\tau \rightarrow \infty$ resultando

$$
U_{i}(Y, \infty)=\sum_{n=1}^{\infty} \varphi_{i, n}(Y) C_{n, i}(\infty) \quad, \quad i=\eta, I
$$

Al resolver el modelo en estado estacionario mediante la Formula de Green se obtiene la siguiente solución

$$
U_{i}^{S}(Y)=\sum_{n=1}^{\infty} \varphi_{i, n}(Y) C_{n, i}^{S} \quad, \quad i=\eta, I
$$


Para garantizar que las soluciones en estado estacionario y transitorio son correctas, se debe demostrar la equivalencia entre (E.6) y (E.7) con base a

$$
C_{n, i}(\infty)=C_{n, i}^{S}
$$

Con ello se puede evaluar el término $C_{n, i}^{T}(\tau)$ de la ecuación (E.2) y la ecuación (E.5) seria

$$
U_{i}(Y, \tau)=\sum_{n=1}^{\infty} \varphi_{i, n}(Y) C_{n, i}^{T}(\tau)+U_{i}^{S}(Y) \quad, \quad i=\eta, I
$$

Pero además se puede obtener la solución analítica directa en estado estacionario para este modelo, que es equivalente al segundo término de la ecuación (E.9); es decir $U_{i}^{S}(Y)=U_{i}^{D}(Y)$, obteniendo la solución compuesta en estado transitorio definida como

$$
U_{i}(Y, \tau)=\sum_{n=1}^{\infty} \varphi_{i, n}(Y) C_{n, i}^{T}(\tau)+U_{i}^{D}(Y) \quad, \quad i=\eta, I
$$

Donde

$$
\begin{gathered}
U_{\eta}^{D}(Y)=U_{\eta}^{1}+\left[\frac{\cosh \left(\sqrt{\Phi_{\mathrm{I}}^{2} \alpha_{\mathrm{I}}^{-1}}\right) U_{\eta}^{1} \kappa_{\eta}}{\cosh \left(\sqrt{\Phi_{\mathrm{I}}^{2} \alpha_{\mathrm{I}}^{-1}}\right) \kappa_{\eta}-\sqrt{\Phi_{\mathrm{I}}^{2} \alpha_{\mathrm{I}}^{-1}} \operatorname{senh}\left(\sqrt{\Phi_{\mathrm{I}}^{2} \alpha_{\mathrm{I}}^{-1}}\right)\left(1-Y_{\eta}\right)}-U_{\eta}^{1}\right] \frac{\left(Y-Y_{\eta}\right)}{1-Y_{\eta}} \\
U_{I}^{D}(Y)=\frac{\cosh \left(\sqrt{\Phi_{\mathrm{I}}^{2} \alpha_{\mathrm{I}}^{-1}} Y\right) U_{\eta}^{1} \kappa_{\eta}}{\cosh \left(\sqrt{\Phi_{\mathrm{I}}^{2} \alpha_{\mathrm{I}}^{-1}}\right) \kappa_{\eta}-\sqrt{\Phi_{\mathrm{I}}^{2} \alpha_{\mathrm{I}}^{-1}} \operatorname{senh}\left(\sqrt{\Phi_{\mathrm{I}}^{2} \alpha_{\mathrm{I}}^{-1}}\right)\left(1-Y_{\eta}\right)}
\end{gathered}
$$

Fase $\eta$

De la ecuación (E.8) se tiene

$$
\begin{gathered}
\frac{-\kappa_{\eta} U_{\eta}^{1}}{\cos \left(\mu_{\eta, n} Y_{\eta}\right) \alpha_{\eta} \mu_{\eta, n}} \frac{1}{D_{n}}=\frac{-\kappa_{\eta} U_{\eta}^{1} \mu_{\eta, n}}{\lambda_{n}^{2} D_{n} \cos \left(\mu_{\eta, n} Y_{\eta}\right)} \\
\frac{1}{\alpha_{\eta} \mu_{\eta, n}}=\frac{\mu_{\eta, n}}{\lambda_{n}^{2}}
\end{gathered}
$$


De la ecuación (E.14) se obtiene la definición dada por la ecuación (2.25) que satisface la relación (E.8) requerida para determinar la solución compuesta. De la ecuación (E.1) se tiene

$$
\begin{gathered}
C_{n, \eta}^{T}(\tau)=\frac{1}{D_{n}} \frac{\kappa_{\eta} U_{\eta}^{1}}{\cos \left(\mu_{\eta, n} Y_{\eta}\right) \alpha_{\eta} \mu_{\eta, n}}\left[e^{-\left(\alpha_{\eta} \mu_{\eta, n}^{2}\right) \tau}-1\right]-\frac{-\kappa_{\eta} U_{\eta}^{1} \mu_{\eta, n}}{\lambda_{n}^{2} D_{n} \cos \left(\mu_{\eta, n} Y_{\eta}\right)} \\
C_{n, \eta}^{T}(\tau)=\frac{\kappa_{\eta} U_{\eta}^{1}}{\cos \left(\mu_{\eta, n} Y_{\eta}\right) D_{n} \alpha_{\eta} \mu_{\eta, n}} e^{-\left(\alpha_{\eta} \mu_{\eta, n}^{2}\right) \tau}-\frac{\kappa_{\eta} U_{\eta}^{1}}{\cos \left(\mu_{\eta, n} Y_{\eta}\right) D_{n} \alpha_{\eta} \mu_{\eta, n}}+\frac{\kappa_{\eta} U_{\eta}^{1} \mu_{\eta, n}}{\lambda_{n}^{2} D_{n} \cos \left(\mu_{\eta, n} Y_{\eta}\right)}
\end{gathered}
$$

Sustituyendo (E.14) en (E.16) se obtiene

$$
C_{n, \eta}^{T}(\tau)=\frac{\kappa_{\eta} U_{\eta}^{1}}{\cos \left(\mu_{\eta, n} Y_{\eta}\right) D_{n} \alpha_{\eta} \mu_{\eta, n}} e^{-\left(\alpha_{\eta} \mu_{\eta, n}^{2}\right) \tau}
$$

Por último al sustituir (E.17) y (E.11) en (E.10) se obtiene las solución compuesta

$$
\begin{aligned}
U_{\eta}(Y, \tau) & =\sum_{n=1}^{\infty} \frac{\sin \left[\mu_{\eta, n}\left(Y-Y_{\eta}\right)\right]}{\cos ^{2}\left(\mu_{\eta, n} Y_{\eta}\right)} \frac{\kappa_{\eta} U_{\eta}^{1}}{D_{n} \alpha_{\eta} \mu_{\eta, n}} e^{-\left(\alpha_{\eta} \mu_{\eta, n}^{2}\right) \tau}+ \\
& U_{\eta}^{1}+\left[\frac{\cosh \left(\sqrt{\Phi_{\mathrm{I}}^{2} \alpha_{\mathrm{I}}^{-1}}\right) U_{\eta}^{1} \kappa_{\eta}}{\cosh \left(\sqrt{\Phi_{\mathrm{I}}^{2} \alpha_{\mathrm{I}}^{-1}}\right) \kappa_{\eta}-\sqrt{\Phi_{\mathrm{I}}^{2} \alpha_{\mathrm{I}}^{-1}} \operatorname{senh}\left(\sqrt{\Phi_{\mathrm{I}}^{2} \alpha_{\mathrm{I}}^{-1}}\right)\left(1-Y_{\eta}\right)}-U_{\eta}^{1}\right] \frac{\left(Y-Y_{\eta}\right)}{1-Y_{\eta}}
\end{aligned}
$$

\section{Fase I}

De la ecuación (E.8) se tiene

$$
\begin{gathered}
\frac{-\kappa_{\eta} U_{\eta}^{1} \mu_{\eta, n}}{\cos \left(\mu_{\eta, n} Y_{\eta}\right)\left(\alpha_{I} \mu_{I, n}^{2}+\Phi_{I}^{2}\right)} \frac{1}{D_{n}}=\frac{-\kappa_{\eta} U_{\eta}^{1} \mu_{\eta, n}}{\lambda_{n}^{2} D_{n} \cos \left(\mu_{\eta, n} Y_{\eta}\right)} \\
\frac{1}{\left(\alpha_{I} \mu_{I, n}^{2}+\Phi_{I}^{2}\right)}=\frac{1}{\lambda_{n}^{2}}
\end{gathered}
$$

De la ecuación (E.20) se obtiene la definición dada por la ecuación (2.25) que satisface la relación (E.8) requerida para determinar la solución compuesta. De la ecuación (E.2) se tiene 


$$
\begin{gathered}
C_{n, I}^{T}(\tau)=\frac{1}{D_{n}} \frac{\kappa_{\eta} U_{\eta}^{1} \mu_{\eta, n}}{\cos \left(\mu_{\eta, n} Y_{\eta}\right)} \frac{1}{\left(\alpha_{I} \mu_{I, n}^{2}+\Phi_{I}^{2}\right)}\left[e^{-\left(\alpha_{I} \mu_{I, n}^{2}+\Phi_{I}^{2}\right) \tau}-1\right]-\frac{-\kappa_{\eta} U_{\eta}^{1} \mu_{\eta, n}}{\lambda_{n}^{2} D_{n} \cos \left(\mu_{\eta, n} Y_{\eta}\right)} \\
C_{n, I}^{T}(\tau)=\frac{\kappa_{\eta} U_{\eta}^{1} \mu_{\eta, n}}{D_{n} \cos \left(\mu_{\eta, n} Y_{\eta}\right)} \frac{e^{-\left(\alpha_{I} \mu_{I, n}^{2}+\Phi_{I}^{2}\right) \tau}}{\left(\alpha_{I} \mu_{I, n}^{2}+\Phi_{I}^{2}\right)}-\frac{\kappa_{\eta} U_{\eta}^{1} \mu_{\eta, n}}{D_{n} \cos \left(\mu_{\eta, n} Y_{\eta}\right)} \frac{1}{\left(\alpha_{I} \mu_{I, n}^{2}+\Phi_{I}^{2}\right)}+\frac{\kappa_{\eta} U_{\eta}^{1} \mu_{\eta, n}}{\lambda_{n}^{2} D_{n} \cos \left(\mu_{\eta, n} Y_{\eta}\right)}
\end{gathered}
$$

Sustituyendo (E.20) en (E.22) se obtiene

$$
C_{n, I}^{T}(\tau)=\frac{\kappa_{\eta} U_{\eta}^{1} \mu_{\eta, n}}{D_{n} \cos \left(\mu_{\eta, n} Y_{\eta}\right)} \frac{e^{-\left(\alpha_{I} \mu_{I, n}^{2}+\Phi_{I}^{2}\right) \tau}}{\left(\alpha_{I} \mu_{I, n}^{2}+\Phi_{I}^{2}\right)}
$$

Por último al sustituir (E.23) y (E.12) en (E.10) se obtiene las solución compuesta

$$
\begin{aligned}
U_{I}(Y, \tau)= & \sum_{n=1}^{\infty} \frac{\sin \left[\mu_{\eta, n}\left(1-Y_{\eta}\right)\right]}{\cos ^{2}\left(\mu_{\eta, n} Y_{\eta}\right)} \frac{\cos \left(\mu_{I, n} Y\right)}{\cos \left(\mu_{I, n}\right)} \frac{\kappa_{\eta} U_{\eta}^{1} \mu_{\eta, n}}{D_{n}} \frac{e^{-\left(\alpha_{I} \mu_{I, n}^{2}+\Phi_{I}^{2}\right) \tau}}{\left(\alpha_{I} \mu_{I, n}^{2}+\Phi_{I}^{2}\right)}+ \\
& \frac{\cosh \left(\sqrt{\Phi_{\mathrm{I}}^{2} \alpha_{\mathrm{I}}^{-1}} Y\right) U_{\eta}^{1} \kappa_{\eta}}{\cosh \left(\sqrt{\Phi_{\mathrm{I}}^{2} \alpha_{\mathrm{I}}^{-1}}\right) \kappa_{\eta}-\sqrt{\Phi_{\mathrm{I}}^{2} \alpha_{\mathrm{I}}^{-1}} \operatorname{senh}\left(\sqrt{\Phi_{\mathrm{I}}^{2} \alpha_{\mathrm{I}}^{-1}}\right)\left(1-Y_{\eta}\right)}
\end{aligned}
$$


USO DE LAS CONDICIONES DE SALTO PARA EL MODELADO DE PROCESOS DE REACCION Y

SEPARACION INTEGRADOS

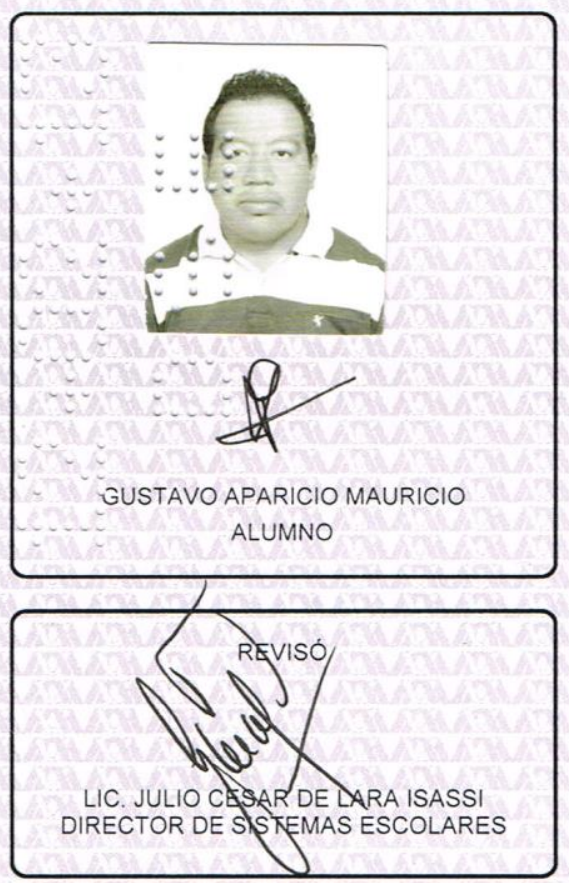

de acuerdo con el artículo 78 fracción III del Reglamento de Estudios Superiores de la Universidad Autónoma Metropolitana, los miembros del jurado resolvieron:

$$
\text { Aprobar }
$$

Acto continuo, el presidente del jurado comunicó al interesado el resultado de la evaluación y, en caso aprobatorio, le fue tomada la protesta.

En México, D.F. se presentaron a las 11:00 horas del día 8 del mes de febrero del año 2013 en la Unidad Iztapalapa miembros del jurado:

DR. HUGO JIMENEZ ISLAS

DR. ALFONSO MAURICIO SALES CRUZ

DR. JOSE DE JESUS ALVAREZ RAMIREZ

Bajo la Presidencia del primero y con carácter de ecretario el ultimo, se reunieron para proceder al Examen obtención del grado de:

MAESTRO EN CIENCIAS (INGENIERIA QUIMICA)

DE: GUSTAVO APARICIO MAURICIO

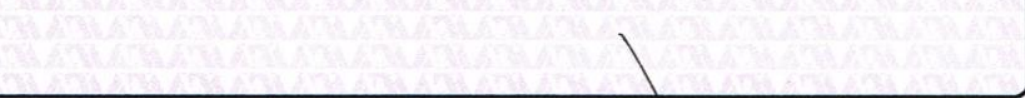

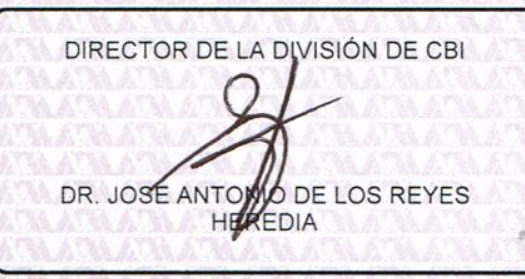

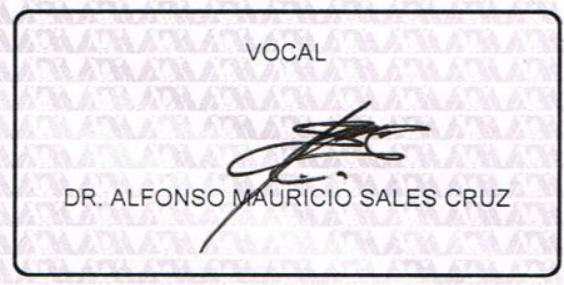

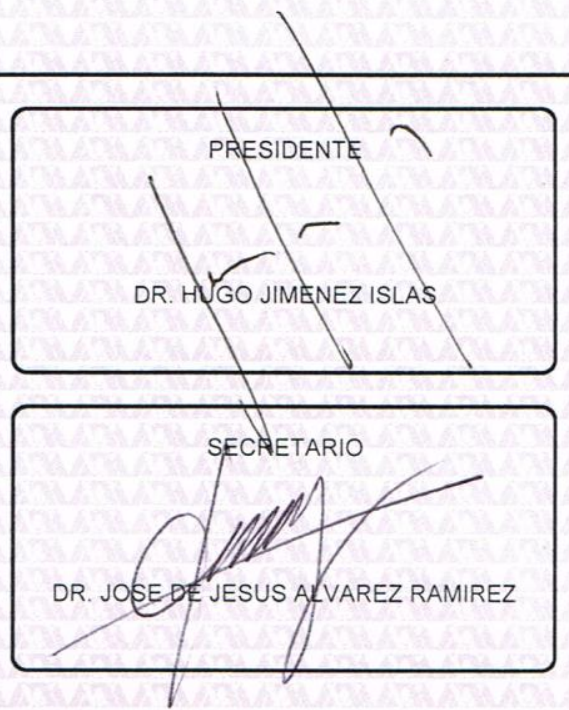

